\title{
Social-Media-Analysen in der Automobilindustrie
}

Die explorative Untersuchung der angewandten Praxis von Social-MediaAnalysen des deutschen Automobilherstellers hat umfassende Ergebnisse hervorgebracht; einen ersten Teil stellt dieses Kapitel als Social-Media-Analysen in der Automobilindustrie vor. Zwei Fragestellungen lagen der Studie zugrunde: Zum einen war von Interesse, was die Praxis von Social-Media-Analysen für die Automobilindustrie und deren Wissen wie Wissensgenese bedeutet; zum anderen galt es die Wissensverwendung zu rekonstruieren und die Beweggründe für die Umsetzung von Social-Media-Analysen zu untersuchen, um zu zeigen, welche Wissenstypen die Automobilindustrie im Kontext von sozialen Medien erzeugt. Im Fokus dieses Kapitels steht die Praxis von Social-Media-Analysen als eine Methode der organisationalen Wissensgenese.

Weder die Frage nach der organisationalen Wissensgenese in der Automobilindustrie noch die nach der Erzeugung von Wissenstypen im Zusammenhang mit Social-Media-Analysen wurde bisher beleuchtet. Dies kann auf zwei Ursachen zurückgeführt werden: Zum einen handelt es sich bei der Automobilindustrie, auch wegen ihres streng gehüteten, Geheimwissens', um einen hermetisch abgeriegelten Industriezweig, in dem nur selten Forschung, welche nicht naturwissenschaftlicher Art und auf die Optimierung von Produkten oder aber die Hervorbringung automobiler Innovationen ausgerichtet ist, realisiert werden kann. Zum anderen besteht zwischen der Automobilindustrie und den Sozialwissenschaften im Allgemeinen oder auch der Wissenssoziologie im Speziellen keine langandauernde, weit in die Vergangenheit zurückreichende Beziehung, wie diese etwa in Form dauerhafter oder entsprechend aktueller Bedarfe wiederholt initiierter Forschungskooperationen mit den Naturwissenschaften gepflegt wird. Daher kann es fast als Novum angesehen werden, dass ein deutscher Automobilhersteller seine Pforten für die Wissenssoziologie geöffnet und eine explorative Untersuchung des ,hinter seinen Mauern * im Kontext von Social-Media-Analysen generierten Wissens sowie der dadurch erzeugten Wissenstypen ermöglicht und unterstützt hat. 
Antworten auf einen Teil der dieser Forschungsarbeit zugrunde liegenden Fragestellung gibt dieses Kapitel. Zunächst wird die angewandte Praxis von Social-Media-Analysen, wie von den praktischen Anwendern dargelegt, deskriptiv zusammengefasst. Daraufhin werden Social-Media-Analysen als Methode behandelt. Abschließend richtet sich der Blick auf die Thematisierung von SocialMedia-Analysen sowohl in ihrer Beziehung zu anderen Methoden als auch im Vergleich mit diesen.

\subsection{Angewandte Praxis von Social-Media-Analysen}

Social-Media-Analysen sind eine Methode, welche im Rahmen der organisationalen Wissensgenese eigesetzt werden kann. Dieser Abschnitt thematisiert die angewandte Praxis von Social-Media-Analysen, wie praktische Anwender des Automobilherstellers sie beschrieben haben. Ein erster Schritt zeigt verschiedene Arten von Social-Media-Analysen, die das betrachtete Unternehmen zum Einsatz bringt. Es geht darum, wie die Befragten diese selbst praktisch durchgeführt oder begleitet haben. Im Anschluss daran wird die konkrete Praxis von SocialMedia-Analysen nachgezeichnet, wie sie in der Automobilindustrie zum Tragen kommt. Beides geschieht anhand der deskriptiven Berichte über die praktische Durchführung.

\subsubsection{Zwei Varianten der Methode}

Im Zuge der theoretischen Betrachtung von Social-Media-Analysen wurde bereits die Unterscheidung von Social Media Listening und Social Media Monitoring behandelt. ${ }^{1}$ Anhand des empirischen Materials greift der vorliegende Abschnitt die zwei Varianten der Methode erneut auf, da die Automobilindustrie beide einsetzt.

Wie die Literatur zu Social-Media-Analysen zeigen die Ausführungen der befragten Social-Media-Analysten keine eindeutige methodische Differenzierung zwischen den zwei Varianten der Methode. Ferner offenbart sich, dass es an einem strukturierten Überblick über die verschiedenen Arten von Social-MediaAnalysen mit entsprechend einheitlicher Benennung mangelt und der Einsatz von Social-Media-Analysen ohne eine methodische Reflexion erfolgt:

\footnotetext{
${ }^{1}$ Anstatt der Begriffe „Social Media Listening“ und „Social Media Monitoring“ verwendet diese Arbeit meist die Abkürzungen „Listening“" und „Monitoring“.
} 
Befragter: [. . . ] Und da gibt es aber, wie gesagt, keine einheitliche Methode in dem Sinne. Der eine misst das so, der andere misst das so. Je nachdem mit welcher Agentur ich da vielleicht auch zusammenarbeite, die, äh, bestimmte Informationen da dann halt eben bereitstellt, ähm, ist das halt ein unterschiedliches Format und es ist auch schwierig miteinander halt zu vergleichen (I14/ S2/ Z44-47).

Es scheint den Praktikern zwar bewusst zu sein, dass keine eindeutige methodische Einordnung der Ansätze vorliegt, jedoch akzeptieren sie diesen Umstand meist ohne ihn zu hinterfragen. Dies mag auch darin begründet liegen, dass im Kontext des praktischen Einsatzes der Methode schlicht keine Ressourcen für methodologische Überlegungen, wie diese die Wissenschaft leistet, vorgesehen sind. So wird die nicht eindeutige methodische Unterscheidung der Varianten von Social-Media-Analysen teilweise zwar erkannt, in der Regel jedoch nicht weiterverfolgt, sondern im praktischen Umgang mit der Methode vernachlässigt oder bewusst ignoriert. Selbst in Fällen, in denen die Befragten explizit zwischen zwei Ausprägungen unterscheiden, bedeutet dies noch keine Eindeutigkeit im Hinblick auf die dahinter liegende Methodik:

Befragter: [. . . ] Und zwar das eine ist für uns immer so das Thema, ähm, Listening. Also das verstehen wir so in diesem klassischen Online Monitoring. Das heißt, wir schauen, Tool basiert, aber auch zum Teil in einer intellektuellen Recherche, ähm, was passiert denn um [Marke des Unternehmens des Befragten] herum auf anderen Plattformen? Ja. Wann schreiben wo Menschen über uns? [. . . ] Und das andere ist mehr so die klassische Analyse des Tracking. Halt, dass man auf Basis der Zahlen, die wir auch, ähm, haben, anhand der KPIs, die wir halt messen, wie/ wie viele Likes, wie viele Interaktionen, wie viel Reichweite, wie viel Conversions, ähm, wird wahrscheinlich noch eine Frage dann auch in der Folge sein. Ähm, ähm, einfach/wie können wir den Status quo optimieren und verbessern? Ja, also das sind so die zwei Stoßrichtungen, die wir sehen und mit denen wir hauptsächlich auch zusammenarbeiten (I6/ S3/ Z9-28).

Mit dieser Aussage setzt ein Praktiker Monitoring gewissermaßen mit Listening gleich; zugleich benennt er eine wesentliche Differenzierung zwischen den zwei Arten der Methode. So ziele die eine Variante eindeutig auf ein ,,Tracking“" und damit auf die „Beobachtung“ oder das Messen einzelner Inhalte sozialer Medien, wohingegen bei der anderen Ausprägung das ,Zuhören“, ,Listening“"genannt, im Mittelpunkt stehe. Die Unterscheidung zwischen Monitoring und Listening, wie sich diese inzwischen auch in der Literatur als eine Gegenüberstellung quantitativer und qualitativer Untersuchungen findet, scheint dem betrachteten Unternehmen noch unbekannt zu sein. Methodische Kenntnisse auf Seiten der Praktiker oder eine reflektierte Auseinandersetzung mit den Verfahren könnte allerdings eine eindeutige Differenzierung zwischen beiden Varianten und damit auch ihre gezieltere Anwendung innerhalb der deutschen Automobilindustrie begünstigen. 
Jeweils ein grundlegender Unterschied sowie eine Gemeinsamkeit von Monitoring und Listening werden in manchen Interviews aber dennoch gezogen und auch eindeutig benannt. Diese sollen, bevor dann beide Varianten näher beschrieben werden, nicht unerwähnt bleiben. So kommt laut dem Datenmaterial Listening für Sonderanalysen oder Ad-hoc-Analysen zu kurzfristig aufgekommenen Fragestellungen und speziellen Anfragen zum Einsatz, Monitoring hingegen für kontinuierliche Messungen oder sich regelmäßig wiederholende Berichterstattungen, wie sie etwa der Unternehmensbereich für Marketing durchführt. Eine Gemeinsamkeit, welche beiden Varianten zugesprochen wird, besteht darin, dass sowohl Monitoring wie auch Listening zwar Direktaussagen der Nutzer sozialer Medien erheben, das Vorgehen von Social-Media-Analysen, gleich welcher Art, aber oftmals mit dem „Lesen einer Glaskugel“ oder dem „Lesen einer Kristallkugel" verglichen wird.

Des Weiteren berichten die Befragten, dass im Kontext von Produktneuanläufen Social-Media-Analysen als ,integrierter Sensor“ genutzt und bewusst in einer Kombination aus Monitoring und Listening eingesetzt werden. Es gehe zum einen darum, Einführungen von Produkten ,,mitzulesen“ und zu erfahren, worüber in deren Kontext „gesprochen“ wird. Obwohl es sich auf den ersten Blick um ein Listening handelt, so ist mit dem kontinuierlichen „Mitlesen“ über einen „Sensor" eine Messung oder Überwachung gemeint, so dass es doch ein Monitoring ist. Zum anderen werden im Zusammenhang der Neuanläufe von Produkten tiefgehende Analysen, sogenannte „Tiefenanalysen“, durchgeführt. Die Begleitung von Produkteinführungen durch Social-Media-Analysen, die auch „Launch Monitoring" genannt wird und durch ihre "offene“ Herangehensweise gekennzeichnet ist, ziele darauf ab, zu „beobachten“ was auf Social-Media-Plattformen geschieht oder auch „Zuzuhören“ worüber dort „gesprochen“ wird; „Tiefenanalysen“ seien im Gegensatz dazu geschlossener und würden zu konkreten, vorab festgesetzten Fragestellungen praktiziert:

Befragter: //Also bei diesen Tiefenanalysen haben wir eine ganze konkrete Fragestellung, ähm, auf, meistens auf Komponenten- oder Funktions-Ebene [. . . ]. Beim, äh, beim Fahrzeug-Monitoring gehen wir eher offen $(. .$.$) rein und sagen halt, ,jetzt$ schauen wir mal, was denn kommt zu diesem Fahrzeug", ja, und da, da wollen wir gar nicht zu viel in die Richtung, ähm, (...) vorgeben, um möglichst, ähm, wirklich auch zu schauen, worüber wird viel oder wenig gesprochen und dann auch eher so Themenfelder erstmal, ähm, zu erkennen, wie ist es eher. [. . . ] Aber da ist da Vorgehen eher nicht/ nicht zu konkret, damit wir ein möglichst gutes Feedback auch rauskriegen, aber bei diesen Tiefenanalysen haben wir immer eine ganz konkrete Fragestellung und wollen dann einfach die Kundenwahrnehmung zu dieser Fragestellung wissen (I5/ S12-13/ Z33-8). 
Es bleibt festzuhalten, dass anhand des Untersuchungsmaterials oft keine eindeutige Abgrenzung zwischen Monitoring und Listening auszumachen ist. Bei beiden Varianten der Social-Media-Analyse geht es letztlich um die Ermittlung von Detailinformationen, um die Erzeugung von Verständnis. In Abhängigkeit von Fragestellung und Intention kann dies auf unterschiedlichen Ebenen und entweder mit dem Fokus auf quantitative oder aber auf , qualitative' Ergebnisse geschehen. Obwohl also aus dem untersuchten Datenmaterial meist nicht eindeutig hervorgeht, welche Art von Social-Media-Analyse im jeweiligen Fall eingesetzt wird, wie dies über eine etablierte Benennung und eindeutige methodische Unterscheidung möglich wäre, kann anhand der beschriebenen angewandten Praxis in den meisten Fällen dennoch eine Zuordnung zu einer Variante erfolgen, wie anhand des Themas „Launch Monitoring“ demonstriert.

\subsubsection{Social Media Monitoring}

Wesentliches Merkmal der Durchführung von Social-Media-Analysen als Social Media Monitoring ist laut den Ausführungen der Befragten deren quantitative Ausrichtung. Wie bereits erwähnt, zielt Monitoring als Variante von Social-MediaAnalysen zunächst auf eine Messung der Inhalte von Social-Media-Kanälen; von der Durchführung werden quantitative Ergebnisse in Form quantifizierter Inhalte sozialer Medien erwartet. Die Befragten haben verschiedene Anwendungsfälle und Praxisbeispiele von Monitoring in der Automobilindustrie berichtet.

Generell setzt der Automobilhersteller Monitoring als Instrument zur „Beobachtung“" seiner ,Außenwelt" ein oder nutzt es als ein Instrument, das überhaupt erst den Blick nach „draußen“ eröffnet und erlaubt. Ist von „draußen“ die Rede, meinen die Befragten außerhalb der Automobilindustrie oder außerhalb ihres Unternehmens. Monitoring werde eingesetzt, um Social-Media-Kanäle systematisch zu durchsuchen, wobei die Variante als ein verhältnismäßig schneller und unkomplizierter Blick nach „draußen“ angesehen wird. Es geht bei Monitoring darum, in hoher Geschwindigkeit und mit nur geringem Aufwand Wissen in das eigene Unternehmen , hineinzuholen'. Oft sprechen die Befragten in diesem Kontext auch von einem ,Sensor“, der den außerhalb des Unternehmens oder der Automobilbranche schlagenden ,Puls“ misst:

Befragter: [. . . ] Und da glaube ich, ist auch Social Media ein super Finger (klopft auf den Tisch) am Puls des Kunden. Ja, wo man sagt, „was ist denn da draußen?“, ja. „Wo geht die Reise hin; was finden die Leute jetzt toll?“, ja. „Was haben sie vor fünf Jahren toll gefunden und was finden sie vielleicht nächstes Jahr toll?"“ und auch mal Prognosen aufbauen. Das ist nämlich so auch die Kür, was wir jetzt gerade mal machen, Prädiktion, ja. Predictive Analytics (I25/ S16/ Z22-27). 
Eben erwähnten „Puls“ beschreiben die Social-Media-Analysten als Konglomerat der Meinungen von Kunden und Nutzern sozialer Medien, die außerhalb des Unternehmens existieren und Themen von Relevanz betreffen, zu denen es keinen anderen Zugang als denjenigen über soziale Medien gibt. So kommt Monitoring auch als ein Instrument zur Ermittlung von Stimmungsbildern zum Einsatz. Insbesondere im Zusammenhang mit Innovationsmanagement und ,Trendscouting ‘ ist diese Nutzung von Monitoring und dessen Einsatz von Bedeutung.

Zudem wird Monitoring genutzt, um „mit dem Ohr am Kunden zu sein“. Durch die Methode würden "Schlaglichter“ erhoben. Mithilfe des Verfahrens lasse sich zum einen die eigene „Performance“ im Sinne von Eigenleistung optimieren und zum anderen in Erfahrung bringen, welche Inhalte auf SocialMedia-Kanälen überhaupt besprochen würden. Monitoring wird zu diesem Zweck insbesondere von Seiten des Marketings eingesetzt. Unter den Bezeichnungen „Social Media Buzz Monitoring“ und auch „Social Media Tracking“ nutzt das Marketing Social-Media-Analysen in der Variante des Monitorings des Weiteren, um eigene Aktivitäten auf Social-Media-Kanälen anhand von Key Performance Indicators (KPIs) zu analysieren. Auch hier besteht das Ansinnen in einer Messung der Eigenleistung. Ferner wird im Kontext des „Buzz Monitoring“ oder „Buzz Tracking“ erneut untersucht, auf welchen Social-Media-Plattformen welche Inhalte diskutiert werden. Monitoring wird von Seiten des Marketing-Bereichs zudem für die Verfolgung eigener Kampagnen und Webseiten und damit auch für deren Überwachung oder Überprüfung im Hinblick auf Gestaltung und Erfolg eingesetzt. Im Fokus einer derartigen Analyse können ferner Veranstaltungen wie Automobilmessen stehen. Auch dieser Anwendungsfall des Trackens von Kampagnen, Webseiten und Veranstaltungen dient dem Ziel der Überprüfung des eigenen Handelns, der eigenen Leistung anhand von Messungen und ist damit Erfolgskontrolle via Quantifizierung. Nebenbei wird Monitoring von manchen Praktikern des Marketings auch für gezielte Recherchen in sozialen Medien verwendet. Dies geschieht allerdings nicht im Rahmen einer kontinuierlich stattfindenden Analyse, sondern nach Bedarf zu spezifischen Ad-hoc-Fragestellungen. ${ }^{2}$

Von einem weiteren Anwendungsfall des Monitorings haben Befragte aus dem Bereich für Kommunikation berichtet. Hier werden Social-Media-Analysen in der Ausprägung des Monitorings einerseits als Element einer umfassenden Medienresonanzanalyse und andererseits für ein Tracking in ,Echtzeit' genutzt. Ersteres heißt,

\footnotetext{
${ }^{2}$ Key Performance Indicators sind Leistungskennzahlen; anhand dieser wird der Fortschritt im Hinblick auf bestimmte Zielsetzungen in einer Organisation gemessen. Siehe hierzu: Franceschini, Galetto, \& Maisano, 2007; Parmenter, 2015.
} 
dass über Monitoring relevante Inhalte von Social-Media-Plattformen ermittelt werden und als solche in allgemeine Medienresonanzanalysen einfließen, die vorrangig Elemente klassischer Medien, aber auch Onlinemedien aus einem feststehenden „Leitmedien-Set“" umfassen. Die Ergebnisse der Medienresonanzanalyse werden in einem monatlichen Rhythmus als Statistik einem reglementierten Empfängerkreis zur Verfügung gestellt. Das Tracking in ,Echtzeit‘, welches Monitoring leistet, wird demgegenüber für einen Medienspiegel beziehungsweise Media Monitor durchgeführt. In diesen mehrmals täglich erstellten und aktualisierten Medienspiegel, der einem eingeschränkten Kreis von Empfängern zugesandt wird, fließen aktuelle, für die Automobilindustrie oder den betrachteten Automobilhersteller relevante Themen, die die Medien thematisieren. Vor allem über Monitoring werden in diesen Medienspiegel „Momentaufnahmen“ der sozialen Medien beziehungsweise der in diesen diskutierten Themen beinahe in ,Echtzeit' integriert. Dabei handelt es sich zumeist um Inhalte, deren Kenntnis wegen der Digitalität der Öffentlichkeit, die mit sozialen Medien einhergeht, als relevant eingestuft werden und die im Hinblick auf die eigene Kommunikationsstrategie zu beachten sind. Außerdem werden Fragestellungen, die für das Unternehmen durchgehend von Bedeutung sind, mit Monitoring verfolgt. Eine Integration von Monitoring in die Kommunikation des Unternehmens sei erforderlich:

Befragter: [... ] Das ist, glaube ich, in Zeiten/, ich bin kein Freund davon, aber so ist nun mal die Welt, in der sich, (stockt) äh, (stockt) sozusagen, in der wir mehr innerhalb von 24 Stunden immer mehr tun können als die Zeiten vorher, wird es immer wichtiger in kürzeren Zeiten, äh, (stockt) zu einer Lageeinschätzung zu kommen. So, und dafür sind soziale Medien geeignet. Sie sind nicht für langfristige/, meine Meinung persönlich, langfristige/, heute nicht für langfristige Strategieableitungen geeignet. Aber sie sind für eine Momentan-Aufnahme, äh, hervorragend geeignet// (I22/ S15/ Z42-48).

Eine Anwendung von Monitoring durch den Unternehmensbereich für Kommunikation lässt sich als „Beobachtung“ oder Überwachung sozialer Medien zusammenfassen.

Wie aus dem Datenmaterial hervorgeht, wird in dem Unternehmensbereich für Qualität das Monitoring ferner als ,Sensor“ zu einer möglichst frühen Erkennung von Fehlern und Beanstandungen an Produkten und Dienstleistungen eingesetzt. Monitoring wird insbesondere genutzt, um die Entwicklung der Neuanläufe in unterschiedlichen Märkten zu verfolgen. Kommt Monitoring im QualitätsBereich als „Sensor“ zum Einsatz, so handelt es sich in erster Linie um ein ,Krisen-Monitoring“, das die Befragten „Early Warning System“ nennen. Auch dieser Anwendungsfall von Monitoring ist als Beobachtung sozialer Medien in Bezug auf die darin geführten Diskussionen über automobile Produkte und Dienstleistungen oder Unternehmen der Automobilindustrie einzustufen. 
Der hier nun abschließend aufgeführte Anwendungsfall von Social-MediaAnalysen in der Ausprägung des Monitorings wird von Praktikern aus dem Unternehmensbereich für F\&E beschrieben. Monitoring ist Ersatz für klassische, etablierte Marktforschung und kommt gemäß dem Untersuchungsmaterial zum Einsatz, um benötigtes Kundenwissen als Wissen von und über Kunden sowie deren Wünsche, Anliegen und Bedürfnisse in Eigenregie zu ermitteln. Hierbei handle es sich meist um Detailinformationen, wie sie für die Fragestellungen des F\&E-Bereichs zwar immer wieder erforderlich sind, für gewöhnlich von den etablierten und offiziellen Marktforschungsstudien aber nicht generiert werden. Sofern die Fragestellungen nicht ausreichend beantwortet würden oder noch Unklarheit zu wesentlichen Themen bestehe, werde Monitoring als eigene Studie, selbstständig und vor allem ohne Abhängigkeit von der für Marktforschung zuständigen Abteilung des Unternehmens durchgeführt.

Es ist festzuhalten, dass in den beschriebenen Anwendungsfällen in erster Linie das Ziel verfolgt wird, anhand von quantitativen Ergebnissen Antworten auf zugrunde liegende Fragestellungen zu finden und ferner Detailinformationen zu ermitteln. Der Fokus liegt auf einer Quantifizierung der Inhalte sozialer Medien. Die über Monitoring ermittelten Ergebnisse sind quantitativer Art und dienen der Beantwortung von Fragestellungen und damit auch der allgemeinen Generierung von Verständnis. Social Media Monitoring ist für die Praktiker ein „Beobachten“ sozialer Medien.

\subsubsection{Social Media Listening}

Im Gegensatz zu Social Media Monitoring zeichnet sich Social Media Listening durch eine ,qualitative' Ausrichtung aus. Mit Listening als Variante von Social-Media-Analysen wird wie mit Monitoring das Ziel verfolgt, Verständnis zu generieren. Im Gegensatz zu Monitoring geht es beim Listening jedoch nicht um eine Quantifizierung der Inhalte sozialer Medien, sondern um das Ermitteln von „detaillierten“ Informationen zu verschiedenen Themen und Fragestellungen sowie um deren Beantwortung anhand einzelner Fallbeispiele. Die praktischen Anwender haben diverse Anwendungsfälle für Listening in ihrem Unternehmen geschildert. Listening ist eine zum Monitoring alternative Möglichkeit. In Kombination zeichnen die im Datenmaterial enthaltenen Ausführungen $\mathrm{zu}$ Monitoring und Listening ein umfassendes Bild davon, welche Varianten von Social-Media-Analysen der betrachtete deutsche Automobilhersteller zur Anwendung bringt. 
Der erste Anwendungsfall wurde ähnlich in Bezug auf Monitoring beschrieben. So wird auch Listening zur „Beobachtung“ der ,Außenwelt" beziehungsweise des Geschehens außerhalb des eigenen Unternehmens und der Automobilindustrie eingesetzt. Listening, so erklären Befragte, eröffne oftmals überhaupt erst die Möglichkeit den Blick nach „draußen“ zu richten und Geschehnisse, welche nicht in der Automobilindustrie oder dem eigenen Unternehmen stattfinden, wahrzunehmen. Daher wird Listening auch mit einem Blick nach „draußen“ gleichgesetzt. Kommt Listening zur Anwendung, dann zumeist mit der Intention, herausfinden $\mathrm{zu}$ wollen, was über das eigene Unternehmen oder die Automobilindustrie in sozialen Medien geäußert wird und auf welche Art dies geschieht. Es geht bei Listening also nicht darum, Informationen in quantitativer Form zu erhalten, sondern um die Ermittlung der auf Social-Media-Plattformen diskutierten Themen und die „Beobachtung“ der Entwicklung dort stattfindender Diskussionen:

Befragter: [. . . ] UND, nichts wirklich Verlässliches triffst du da im Netz, ne. Also, ABER du kriegst eine Diskussion mit, du merkst (stockt) eine Strömung, eine Richtung (I16/ S3/ Z11-12).

Die befragten Social-Media-Analysten schätzen Listening als eine ,qualitative Methode ein, mit der Themen und ihre Diskussion sowie die davon ausgehenden oder damit verbundenen Tendenzen zu ermitteln sind. Ist von „Strömungen“ oder „Richtungen“ die Rede, verweist dies aber auch darauf, dass mit Listening nach dem Verständnis des betrachteten Unternehmens keine verlässlichen Messungen umgesetzt werden, die eindeutige Ergebnisse hervorbringen. Vielmehr sei Listening nur dazu in der Lage, Tendenzen zu ermitteln. Mit dieser Einordnung von Listening sprechen die Befragten dem Verfahren eine Orientierungsfunktion zu. Diese ermögliche es, Themen als relevant zu erkennen. Damit leiste Listening Orientierung hinsichtlich der zahlreichen außerhalb der Automobilindustrie oder des eigenen Unternehmens, in sozialen Medien diskutierten Themen. Durch Listening würden solche Inhalte in eigene Überlegungen integriert oder aus diesen ausgeschlossen. Listening schaffe Orientierung zur Lenkung der eigenen Aufmerksamkeit, die für den Automobilhersteller auch nur beschränkt sein kann und daher bewusst gesteuert werden muss. Jedoch wird Listening - das darf an dieser Stelle nicht verschwiegen werden - von den Befragten oft degradiert und als unbedeutend im Hinblick auf den unternehmerischen „Mehrwert“ eingestuft, weil es der Ermittlung einer ersten Orientierung über, qualitative" Forschung diene und anders als die quantifizierende Herangehensweise des Monitorings keine konkreten oder direkt greifbaren Ergebnisse in Form von „Zahlen, Daten, Fakten“ liefere. 
Trotzdem ist dem Datenmaterial zu entnehmen, dass Listening einen zentralen Beitrag zum Verständnis der, Außenwelt ' leistet. Die Befragten sehen den Ansatz als Verfahren an, das heutzutage schlichtweg zur Anwendung gebracht werden muss. Es bestehe gegenwärtig eine Notwendigkeit zur Nutzung von Listening; an Listening führe kein Weg mehr vorbei. Ebenso wie der Zwang zu Monitoring wird diese Unumgänglichkeit des Einsatzes von Listening aus der mit sozialen Medien verbundenen Öffentlichkeit beziehungsweise deren digitaler Qualität abgeleitet. Verstärkt wird die Einschätzung einer unbedingten Notwendigkeit der Anwendung von Listening durch die Erkenntnis, dass die Kommunikation in sozialen Medien, die auch eine neue Art der Öffentlichkeit markieren, stark zugenommen habe. Hinzu kommt die Annahme einer noch weiteren Steigerung in der Zukunft. Listening stufen die Befragten als eine für ihr Unternehmen und die deutsche Automobilindustrie insgesamt erforderliche Ausprägung von Social-Media-Analysen ein. Dies nicht zuletzt, da die ,qualitative" Ausrichtung des Verfahrens bedeutet, den auf Social-MediaPlattformen geführten Diskussionen ,zuzuhören“ und diese nicht in Quantitäten abzubilden. Oftmals ermöglichen Detailinformationen, die über Listening gewonnen werden, erst Verständnis in Bezug auf Fragestellungen. Daher wird dem Verfahren ein zentraler Stellenwert zugesprochen. Allerdings - dies ist hinsichtlich der erwähnten Degradierung von Listening im Vergleich zu Monitoring zu ergänzen - scheint der Automobilhersteller bislang weder versiert im Umgang mit noch der Einordnung von, qualitativen' Erkenntnissen.

Die Ermittlung einzelner Diskussionen unter Nutzern sozialer Medien geschieht im Kontext von Listening über ein „Zuhören“. Berichte erinnern an das der qualitativen Sozialforschung entstammende Verfahren einer nicht-teilnehmenden Beobachtung. Listening wird als ein passives Verfahren angesehen, welches es möglich macht, die Nutzer von sozialen Medien zu ,,beobachten“, diesen „,zuzuhören“"und ihre Äußerungen ,,mitzulesen“ ohne selbst als Beobachter, Zuhörer oder Leser bemerkt zu werden. So könnten wertvolle Informationen darüber gewonnen werden, was auf Social-Media-Plattformen bezüglich der Automobilindustrie oder des eigenen Unternehmens, seiner Marken, Produkte und Dienstleistungen sowie in Bezug auf spezifische Fragestellungen diskutiert wird. Einzelne Themen könnten dann wiederum hinsichtlich ihrer Bedeutung und Relevanz für die Nutzergemeinschaft von Social Media besser eingeordnet werden:

Befragter: //Mit dem Ohr am Kunden sein. Oder am Interessenten. Listen/ Social Media/ Social Media Listening, genau (I12/ S3/ Z3-4).

Beim Listening geht es darum, mitzubekommen, worüber in sozialen Medien ein Austausch stattfindet: wie stark, mit welcher Konnotation und in welchem Kontext. 
„Netnographie“ ist eine alternative Bezeichnung für Listening als Variante von Social-Media-Analysen. Sofern der Begriff gewählt wird, geht dieser auf eine bestimmte für die Durchführung von Social-Media-Analysen beauftragte Agentur zurïck, welche ihr Listening unter dieser Bezeichnung vermarktet. Auch dabei werden Informationen zu im Vorfeld festgesetzten Themen auf Social-MediaKanälen erhoben. Wie beim Listening möchten die Befragten dem Austausch in sozialen Medien „zuhören“ oder diesen „beobachten“, um frühzeitig über die Entwicklung im Bilde zu sein. ${ }^{3}$

Wesentliche Beweggründe für die Nutzung von Listening sind die Ermittlung von Frühindikationen, die Generierung von Input sowohl allgemein als auch speziell sowie das Thema „Trendscouting“. Die ,hinter“ sämtlichen Beweggründen stehende Absicht besteht darin, Verständnis zu schaffen:

Befragter: [ . . ] ] [̈̈]hm, da war eben dann schon der Wunsch, ,okay, wir wollen bitte, ähm, in strukturierterer Form noch verstehen, (stockt) womit sich/ womit sich unsere Kunden beschäftigen“ (I32/ S8/ Z15-17).

Ein weiterer konkreter Anwendungsfall, der die Definition dessen mitbestimmt, was unter Listening, gerade auch in Abgrenzung zu Monitoring, verstanden wird, besteht in der Prävention:

Befragter: [. . . ] Sprich, eine Social-Media-Analyse ist/ ist für mich erstmal, ähm, die Defizite der Vergangenheit zu identifizieren und sie in Zukunft auszumerzen. Ähm, ist reaktiv, wenn man es genau betrachtet. Man kann es aber auch, äh, präventiv nutzen (I20/ S5/ Z19-21).

Die Durchführung des Verfahrens und damit Listening als mögliche Variante von Social-Media-Analysen bedeutet, dass in sozialen Medien stattfindende Diskussionen verfolgt und als ,Außensicht" in das eigene Unternehmen ,hineingeholt ${ }^{*}$ werden. Befragte sprechen von „Meinungen“ und „Erfahrungen“ einer breiten Masse, die als Reaktion oder Rückmeldung außerhalb ihres Unternehmens entstanden sind und über Listening in dieses ,hineinfinden'. Daher ist im untersuchten Datenmaterial auch die Rede von der ,reaktiven Entstehung der Inhalte sozialer Medien“ und der „Möglichkeit zu deren präventiver Nutzung“.

3 „Netnographie“ ist ein von Kozinets (2010) geprägtes Kunstwort, das die Begriffe „Internet“ und „Ethnographie“ verbindet; darunter wird die Übertragung ethnographischer Forschungsmethoden auf das Internet diskutiert. Siehe hierzu auch: Janowitz, 2014. 
Ein weiterer von den Praktikern thematisierter und hier vorerst abschlieBend genannter Aspekt, der Listening als Variante von Social-Media-Analysen beschreibt, besteht in der Nähe des Verfahrens zu Marktforschung. Bislang wurden Meinungen von „draußen“ vorrangig durch Marktforschung in das Unternehmen ,hineingespült". Marktforschung würde zwar weiterhin praktiziert, jedoch um digitale Methoden erweitert. Social-Media-Analysen werden in der Ausprägung des Listenings oftmals als digitale Ergänzung oder auch Möglichkeit beschrieben, die Marktforschung zu begleiten.

Zusammenfassend ist Social Media Listening als ,qualitative" Variante von Social-Media-Analysen zu charakterisieren. Eingesetzt wird das Verfahren, um in Anlehnung an die zur qualitativen Sozialforschung zählende Methode der nichtteilnehmenden Beobachtung der Netzöffentlichkeit unbemerkt, ohne Reaktivitätsprobleme „,zuzuhören“. Listening liefere „detaillierte“ Informationen zu zugrunde liegenden Fragstellungen. Wie Monitoring schafft auch Listening Verständnis und gibt Orientierung. ${ }^{4}$

\subsubsection{Methodische Umsetzung und praktische Durchführung}

Nachdem vorgestellt wurde welche Varianten der Social-Media-Analyse die praktischen Anwender des Unternehmens umsetzen, geht es nun darum, auf welche Weise dies geschieht. Zu diesem Zweck werden die Schilderungen der Befragten über die methodische Umsetzung und praktische Durchführung ihrer Social-MediaAnalysen deskriptiv zusammengefasst. Wie gezeigt, unterscheiden die Praktiker nicht eindeutig zwischen Listening und Monitoring als zwei Varianten von SocialMedia-Analysen. Aus ihren Berichten über die methodische Umsetzung lassen sich jedoch zwei grundlegend unterschiedliche Vorgehensweisen ableiten: Eine pragmatische gegenüber einer zumindest vermeintlich professionellen Variante, wobei Letztere noch einmal in zwei Dimensionen zu unterteilen ist. Alle drei Varianten stellt dieser Abschnitt vor.

Zunächst wird die Durchführung von Social-Media-Analysen als Desk Research, auch „Schreibtischforschung“ genannt, beschrieben. Bei dieser Art der Umsetzung handelt es sich um die eher pragmatische Herangehensweise, die mitunter mit einem bloßen Googeln gleichzusetzen ist, verstanden als Recherche im Internet unter Zuhilfenahme der Suchmaschine Google. Der Fokus einer solchen

4 „Netzöffentlichkeit“ ist ein von Grundwald, Banse, Coenen und Hennen (2006) geprägter Begriff; mit diesem beschreiben sie die Öffentlichkeit des Internets im Kontext digitaler Demokratie. 
als „Desk Research“ bezeichneten Recherche richtet sich in erster Linie auf in sozialen Medien auffindbare Inhalte und klammert weitere über Google ermittelte und damit im Internet verfügbare Ergebnisse aus. Die Befragten nennen diese Art der Durchführung auch ,rein manuelles“ oder ,händisches“ Vorgehen. Für die manuelle Umsetzung werden diverse Beweggründe angeführt. Weil zum einen eine Fokussierung auf die Inhalte sozialer Medien stattfindet und die Praktiker Desk Research zum anderen als Variante der Anwendung von SocialMedia-Analysen darlegen, versteht auch die hier vorliegende Studie diese Art der Durchführung als eine von mehreren möglichen. Als pragmatisch gilt sie, da sie meist wenig standardisiert erfolgt, sondern ad hoc stattfindet. Außerdem suchen damit oft Einzelpersonen in Eigenregie Antworten auf Fragestellungen, die ,auBer der Reihe" aufgekommenen sind. Trotz der meist aus dem Augenblick heraus entstandenen Fragen können durchaus Routinen in der praktischen Anwendung entstehen. 5

Neben der als pragmatisch eingestuften Variante der Durchführung von Social-Media-Analysen mit Desk Research ist eine Umsetzung über Tools oder Dienstleister möglich. Dabei handelt es sich gemäß den Befragten um eine als standardisiert wahrgenommene Art der Vorgehensweise. Diese wird nicht zuletzt wegen ihrer Standardisierung als professionelle Variante der Durchführung eingestuft. Jedoch - dies sei bereits vorab erwähnt - existiert die Professionalität beim Einsatz von Tools oder einer Beauftragung von Agenturen oftmals nur vermeintlich. Professionalität kann nicht per se in einer Standardisierung der Umsetzung begründet sein, sondern kann im Gegensatz dazu auch in einer Routine bei der praktischen Anwendung von Social-Media-Analysen via Desk Research bestehen. Dennoch spricht diese Arbeit von einer „professionelleren“ Variante von Social-Media-Analysen, sofern diese mit Tools oder durch Dienstleister oder aber eine Kombination beider umgesetzt werden. Weiterhin kann die professionellere Variante in zwei Dimensionen unterteilt werden. Gemeint ist, dass eine standardisierte Durchführung zum einen insofern möglich ist, dass diese über professionelle Tools erfolgt, aber nur innerhalb des Unternehmens. Zum anderen können Social-Media-Analysen auch bei Agenturen beauftragt werden, so dass diese die Umsetzung übernehmen und die Ergebnisse an das Unternehmen liefern. Dienstleister - so viel sei vorweggenommen - setzen meist zwar selbst Tools ein, unterscheiden sich allerdings bezüglich der automatisierten gegenüber der manuellen Durchführung bei der Erhebung und Auswertung der Daten erheblich. Die

\footnotetext{
${ }^{5}$ Den Begriff „Desk Research“ verwenden Marketing und Marktforschung für Recherchen im Internet. Siehe hierzu: Nickel, 2004.
} 
Praktiker haben Erfahrungen mit diversen Dienstleistern gesammelt und entsprechend deren Vorgehen geschildert. Allerdings werfen manche Ausführungen die Frage auf, ob diese selbst über Kenntnisse in sozialen Medien und sozialwissenschaftlichen Forschungsmethoden verfügen oder ob eher von deren methodischem und medialem Unwissen auszugehen ist. Wäre Letzteres der Fall, so würde der für die Durchführung der Social-Media-Analysen beauftragten Agentur ein nahezu ,blindes Vertrauen“ entgegengebracht.

Die methodische Umsetzung einzelner Social-Media-Analysen unterscheidet sich also zunächst im Hinblick darauf, ob es sich um ein pragmatisches oder professionelles Vorgehen handelt. Erscheint dieses professionell, wird noch einmal dahingehend unterschieden, ob die Umsetzung in dem Unternehmen über Tools erfolgt oder von einer Agentur vorgenommen wird. Des Weiteren ist es möglich, mehrere Formen der Durchführung miteinander zu kombinieren, so dass eine Social-Media-Analyse zum Beispiel zunächst manuell über Desk Research und im Anschluss daran automatisiert mit einem Tool durchgeführt wird oder aber in einem ersten Schritt manuell über Desk Research, anschließend von einer Agentur und parallel mit einem Tool im Unternehmen selbst. Zudem kann auch im Zeitverlauf ein Wechsel von einer manuellen Durchführung via Desk Research oder der über Tools im Unternehmen umgesetzten Social-Media-Analysen zu Durchführungen über einen Dienstleister erfolgt sein. Außerdem sind Sonderformen von Social-Media-Analysen denkbar. Die Befragten haben von zwei Kooperationen berichtet, einerseits von einer Zusammenarbeit an dem Tool eines Anbieters, andererseits von einem gemeinsamen Projekt mit einer wissenschaftlichen Forschungseinrichtung, um deren Angebot zu erproben. Die geschilderten Varianten der methodischen Umsetzung von Social-Media-Analysen stellt diese Arbeit im Einzelnen nun näher vor. Auch dies geschieht in Form einer deskriptiven Zusammenfassung mit dem Anspruch, diese methodisch so detailliert, wie es das Datenmaterial erlaubt, jedoch auch zu einer der drei Varianten aggregiert zu gestalten.

\subsubsection{Desk Research als Variante von Social-Media-Analysen}

Die Vorgehensweise bei Social-Media-Analysen mit Desk Research gestaltet sich pragmatisch:

Befragter: [. . . ] Gut, und dann bin ich halt selber erstmal ein bisschen, ähm, online gegangen und habe die diversen Foren, Motor-Talk.de und so weiter mir ein bisschen angeschaut (I3/ S1/ Z22-23). 
Diese Art der Umsetzung von Social-Media-Analysen ähnelt einfachem Googeln und erfolgt relativ offen. Der zentrale Unterschied zwischen einer Internetrecherche unter Nutzung der Suchmaschine Google und Desk Research als Variante von Social-Media-Analysen besteht darin, dass sich Letztere auf in sozialen Medien auffindbare Inhalte fokussiert, wohingegen eine Suche über Google oft zunächst ohne Einschränkungen im Hinblick auf die Art der medialen Quellen, die in die Recherche einbezogenen werden, beginnt. Als pragmatisch gilt die Vorgehensweise bei Social-Media-Analysen über Desk Research aus zwei Gründen: Einerseits, da die Umsetzung für gewöhnlich manuell, also ohne Zuhilfenahme von spezifischen Tools, erfolgt; andererseits, da Social-MediaAnalysen über Desk Research in der Regel nur wenig standardisiert umgesetzt und auch nicht regelmäßig praktiziert werden, sondern als Sonderanalysen zu Ad-hoc-Fragestellungen:

Befragter: [. . . ] Beziehungsweise halt in Klein das für uns selber am Rechner gemacht. Jetzt nicht systematisiert [... ] (I28/ S7/ Z39-40).

Die Beweggründe für die Durchführung von Social-Media-Analysen als Desk Research können erheblich variieren.

Einige Befragte haben Social-Media-Analysen in Form von Recherchen über Google betrieben. Solche Social-Media-Analysen führen sie zu spezifischen Fragestellungen durch, für deren Beantwortung eine Ermittlung von Detailinformationen erforderlich ist. Meist stehen bei einem solchen Vorgehen für den je relevanten Markt bestimmte Foren im Fokus, wie etwa Automobilportale, auf denen ein fachlich ,detaillierter“ Austausch zu spezifischen Themen stattfindet. Bei Fragestellungen, die sehr detaillierte Antworten bedürfen, klammern die Befragten bestimmte Social-Media-Kanäle aus, wie Facebook, Pinterest und Twitter sowie allgemeine Foren, und konzentrieren sich stattdessen zum Beispiel auf spezifische Foren, in denen die für ihre Fragestellung relevante Technik diskutiert wird.

Ferner kommen Social-Media-Analysen als manuell durchgeführte Desk Research bei dem betrachteten Automobilhersteller immer wieder zum Einsatz, um die eigenen, aus dem Unternehmen heraus initiierten selbst durchgeführten SocialMedia-Aktivitäten zu „,beobachten“. Beispielsweise hat der für Design zuständige Unternehmensbereich einen eigenen Account auf dem Social-Media-Kanal Facebook angelegt. Dieser wird seitdem selbstständig mit Inhalten „,bespielt“; die dort veröffentlichten Texte und Bilder werden selbst ,produziert“. Der Eröffnung des Accounts ist eine Social-Media-Analyse via Desk Research zur Ermittlung der Bedeutung von sozialen Medien generell und der Relevanz diverser Plattformen 
einschließlich der damit einhergehenden Möglichkeiten für den Unternehmensbereich vorausgegangen; Social-Media-Kanäle wie Facebook, YouTube und Pinterest wurden bewertet. Mit der eignen „Bespielung“ des Accounts auf Facebook sind weitere Social-Media-Analysen verbunden, wobei die „Bespielung“ und auch die Analyse manuell, also ohne den Einsatz von Tools oder die Beauftragung einer Agentur erfolgt und von Mitarbeitern des Design-Bereichs realisiert wird. Einerseits werden also die eigenen Aktivitäten auf Facebook als Selbstbeobachtung und auch die Reaktionen von Social-Media-Nutzern darauf analysiert; andererseits wird der Blick auf externe, immer aber thematisch verwandte Social-MediaKanäle gerichtet. Das eigene, subjektive` Empfinden fließt in die Untersuchungen ein. Zum einen geht es um die Ermittlung von Resonanz, zum anderen um das Aufspüren von Inspiration und Ideen als Input. Eine Betrachtung der Resonanz in sozialen Medien wird „Tonalitätsanalyse“ oder „Sentiment-Analyse“ genannt. Im beschriebenen Beispiel aus dem Bereich Design ist eine Tonalitätsanalyse als Social-Media-Analyse über Desk Research so zu verstehen, dass manuell die Tonalität der Rückmeldungen zu den in sozialen Medien veröffentlichen Beiträgen analysiert wird. Es geht darum, zu ermitteln, welche Art von Beiträgen oder auch welche Inhalte von Wettbewerbern und Vorbildern positive und negative Resonanz erzeugen. Auch die Bewertung eigener Aktivitäten auf Facebook und damit letztlich der Erhalt von Feedback sind von Interesse. Über Social-Media-Analysen wird Orientierung gewonnen, um das eigene Handeln in den sozialen Medien entsprechend auszurichten und in gewisse als relevant erkannte Richtungen zu lenken. Das Vorgehen bei dieser Art von Social-Media-Analysen ist explorativ. Man möchte sehen, welche Aktivitäten auf positive Resonanz stoßen und welche nicht. Zwar werden für die „Beobachtung“ des eigenen Accounts auch die von Facebook bereitgestellten Statistiken betrachtet; da sie jedoch öffentlich für alle Nutzer einsehbar sind, werden sie hier nicht als Tool zur Analyse für soziale Medien, sondern als integrierter Bestandteil dieser Social-Media-Plattform verstanden. Aus diesem Grund ist das vom Design-Bereich praktizierte Vorgehen als ausschließlich manuell durchgeführte Desk Research einzuordnen. Methodisch beinhaltet diese zudem Bestandteile, die mit einer qualitativen Inhaltsanalyse und auch Bildanalyse vergleichbar sind. Zwar werden diese beiden dem Methodenkanon qualitativer Sozialforschung angehörenden Verfahren hier nicht, in sozialwissenschaftlicher Reinform' praktiziert und es ist auch davon auszugehen, dass die Social-Media-Analysten des Bereichs für Design keine fundierten Kenntnisse sozialwissenschaftlicher Methoden besitzen, dennoch weist das beschriebene Vorgehen Anleihen bei beiden Verfahren auf. Entsprechend berichten die Praktiker, dass eine Auseinandersetzung mit den Bildwelten, die von Wettbewerbern oder anderen als Inspirationsquelle eingestuften Marken in sozialen Medien gezeigt 
werden, erfolgt ist. Ziel dieser Untersuchungen der Aktivitäten und Bildwelten von Wettbewerbern sowie Vorbildern ist es, Orientierung für die „Bespielung“ des eigenen Social-Media-Kanals zu erhalten.

Frequenzanalysen finden sich vor allem in quantitativ ausgerichteten und über Tools umgesetzten Social-Media-Analysen, werden vom Unternehmensbereich für Design auch in Social-Media-Analysen über Desk Research integriert und dementsprechend manuell praktiziert. Zum Beispiel fließt die Textlänge der Äußerungen, die in sozialen Medien getätigt werden, in die Betrachtung mit ein. Es handelt sich dabei um eine Frequenzanalyse im weitesten Sinne. Es wird die Textlänge von veröffentlichten Beiträgen verglichen; in den Ausführungen der Befragten bleibt jedoch offen, welche Maßeinheit den Analysen zugrunde liegt. Denkbar wären die Anzahl einzelner einen Beitrag bildender Worte, Zeilen oder Absätze. Des Weiteren werden beispielsweise Uhrzeiten, zu denen Beiträge in sozialen Medien veröffentlicht werden, und die Frequenz der zu diesen Uhrzeiten erhaltenen Rückmeldungen zueinander in Bezug gesetzt. Auf diese Weise sollen die am besten für Veröffentlichungen geeigneten Uhrzeiten ermittelt werden, um das eigene Handeln in Social Media entsprechend anzupassen. Man kann festhalten, dass auch die über Desk Research durchgeführten Social-Media-Analysen des Design-Bereichs auf Orientierung abzielen. Beobachtende Verfahren sollen Orientierung geben. Sozialwissenschaftliches Methodenwissen liegt diesen SocialMedia-Analysen nicht zugrunde. Im Fokus der Untersuchungen steht neben der Selbstbeobachtung ein Vergleich mit dem Wettbewerb und das Aufspüren von Inspirationsquellen und ,Einflussgrößen' sozialer Medien in Gestalt von Nutzern mit hoher Reichweite beziehungsweise Influencern. Durchgeführt werden die Social-Media-Analysen des Unternehmensbereichs für Design rein manuell.

Eine spezifische Form von Social-Media-Analysen über Desk Research stellt deren Umsetzung als Suche anhand der Social-Media-Plattform YouTube dar. Gemäß den Ausführungen der Praktiker handelt es sich dabei ebenfalls um ein manuelles Vorgehen, bei dem YouTube „,von Hand“ oder „hands-on“ nach relevanten Inhalten durchsucht wird; diese sollen die jeweilige Fragestellung beantworten oder einen Beitrag zu deren Beantwortung leisten. Eine solche Recherche über YouTube führen die Befragten selbst, im Unternehmen, ohne Einsatz spezieller Tools durch. Dabei gehen sie explorativ und offen vor, keineswegs standardisiert. Obwohl eine Standardisierung solcher Suchen auf YouTube nicht gegeben ist, finden diese dennoch systematisiert statt, indem diese Variante der SocialMedia-Analyse nach bestimmten, vorab festgelegten Suchkriterien durchgeführt wird. Ein Befragter berichtet davon, dass allen als Suche über YouTube umgesetzten Social-Media-Analysen die gleichen Suchkriterien zugrunde liegen. Im Kontext dieser Suchen nach Ideen für Innovationen werde nach „Highlights“, 
„Nightmares“ und „Work Arounds“ recherchiert zur Erarbeitung dessen, was die Kunden und Nutzer als Mangel bestehender Produkte und als Verbesserungsoptionen diskutieren. Zudem werde nach „Ideas“ für bisher am Markt nicht angebotene, jedoch benötigte Produkte und Dienstleistungen gesucht. Alternativ kann Systematisierung bedeuten, dass gewisse YouTube-Kanäle regelmäßig angesehen werden. Im Kontext der Ermittlung von Ideen für Innovationen würden die YouTube-Kanäle von Tech Crunch und TheVerge verfolgt. Social-Media-Analysen werden als visuelle Untersuchungen beschrieben, mit denen die Praktiker Inhalte von Social Media, die einer Beantwortung offener Fragen zuträglich sein können, ansehen. Ferner gilt die Social-Media-Plattform YouTube als die ,größte und beste Suchmaschine“ im Internet überhaupt. Begründet wird diese Behauptung damit, dass der Konsum visueller Inhalte leichter falle als das Lesen von geschriebenem Text. Visuelle Materialien würden ein besseres Verständnis ermöglichen als dies lange Texte können; Sehen sei einfacher als Lesen. Man kann also festhalten, dass hier ein , visuelles Verstehen' proklamiert wird; dieses wird als über eine Suche mit YouTube und damit über manuell mit Desk Research umgesetzte Social-Media-Analysen realisierbar bewertet. Die Befragten legen Recherchen mit YouTube oft auch als ersten Schritt von Social-MediaAnalysen dar; begründet wird dies mit der Einfachheit des Einstiegs in ein neues, noch unbekanntes Themengebiet über den Konsum visueller, in sozialen Medien vorhandener Inhalte.

Eine weitere im Kontext der mit YouTube durchgeführten Social-MediaAnalysen thematisierte Variante stellt die Verfolgung von Livestreams dar. Zwar werden diese nicht unbedingt nur durch YouTube übertragen, sondern auch über andere Social-Media-Kanäle, bestehen aber ebenso vorrangig aus visuellen Inhalten. Social-Media-Analysen werden von einigen praktischen Anwendern mit der Rezeption visueller Inhalte gleichgesetzt. Das Verfolgen von Liveübertragungen anhand sozialer Medien gilt in dem betrachteten Unternehmen als Variante von Social-Media-Analysen. Beispielhaft werden zur Ermittlung von Innovationen der Livestream der Consumer Electronics Show (CES) in Las Vegas oder Übertragungen großer Pressekonferenzen in sozialen Medien genannt. Mit der Durchführungsvariante über die Plattform YouTube hat eine methodisch spezifische Form von Social-Media-Analysen als Desk Research Eingang in die vorliegende Arbeit gefunden. Diese erinnert an die schon dargestellte Ausprägung von Social-Media-Analysen als Recherche über Google, schließt jedoch wegen der Fokussierung auf einen einzigen Kanal sämtliche weitere Plattformen aus und rückt methodisch die manuelle Ermittlung von Wissen in visueller Form in das Zentrum der Betrachtung. Verglichen mit den Suchen über Google ist das Vorgehen damit deutlich weniger offen, dennoch ebenfalls explorativ. 
Werden Social-Media-Analysen manuell mit Desk Research durchgeführt, so geschieht dies meist entweder über eine Recherche unter Zuhilfenahme der Suchmaschine Google oder über eine gezielte Suche in den der jeweiligen Fragestellung entsprechenden Foren und in sozialen Medien im Allgemeinen. Nicht selten werden beide Möglichkeiten kombiniert oder um die Option der Suche in Pressedatenbanken, ergänzt oder aber sie erfolgen in umgekehrter Reihenfolge. Mit einer manuellen Online-Recherche von in Printmedien publizierten Artikeln, Testberichten und den darauf basierenden, in Social Media daran anknüpfenden Diskussionen schildern die Praktiker eine ohne den Einsatz eines Tools oder die Beauftragung einer Agentur umgesetzte Social-Media-Analyse, die auf Printerzeugnisse von Automobilzeitschriften oder andere für die jeweilige Fragestellung relevante Printmedien fokussiert ist. In Form von Desk Research werden diese ermittelt und mitsamt den anknüpfenden Kommentaren rezipiert. Wagt man einen Vergleich mit sozialwissenschaftlichen Forschungsmethoden, erinnert das Vorgehen vage an eine qualitative Inhaltsanalyse oder an eine Erhebung und Auswertung von Daten anhand der Grounded Theory Methodologie. Auch hier ist anzumerken: Die diese Variante praktizierenden Befragten besitzen keine Kenntnisse sozialwissenschaftlicher Methoden.

Zudem werden Social-Media-Analysen über Desk Research als manuell durchgeführte Tonalitätsanalyse, die in ihrer methodischen Durchführung ebenfalls an eine qualitative Inhaltsanalyse denken lässt, beschrieben. Dies ist auch deshalb der Fall, weil mit Nachdruck darauf hingewiesen wird, dass Erkenntnis nur durch die Kombination von hohem Zeitaufwand mit ,qualitativer' Auswertung in Gestalt einer Aggregation von einzelnen Informationen zu generieren ist. Erneut gilt: Auch diese Praktiker verfügen nicht über fundierte Kenntnisse sozialwissenschaftlicher Methoden.

Social-Media-Analysen über Desk Research werden ferner in Form von Bildanalysen umgesetzt. Dies bedeutet, dass die Praktiker in erster Linie visuelle Inhalte sozialer Medien betrachten und als solche analysieren. Die an die Bilder anschließenden Kommentare beziehen sie in ihre Auswertung ein. In diesem Kontext verweisen die praktischen Anwender vor allem auf die Social-MediaPlattform Instagram, die umfangreiche visuelle Informationen bereithält. Sowohl die Recherche als auch die Auswertung des Bildmaterials erfolgt, wie bei Desk Research üblich, manuell. Oftmals kommt diese Art von Social-Media-Analysen parallel zu deren Durchführung durch Agenturen zum Einsatz. Hinter dieser Doppelung steht das Ansinnen, die Durchführung von Social-Media-Analysen nicht vollständig auszulagern und das dafür nötige Wissen ausschließlich einzukaufen, sondern dieses auch innerhalb des eigenen Unternehmens aufzubauen beziehungsweise zu erhalten. Jedoch ist dadurch wiederum, so geht aus Berichten hervor, ein 
Übergang von Social-Media-Analysen als Desk Research auf eine Umsetzung mit Tools nicht ausgeschlossen; vor allem bei Expansionen und auch dem Wandel der Social-Media-Analysen von einem operativen zu einem konzeptionellen Thema.

Es ist festzuhalten, dass diese methodische Umsetzung von Social-MediaAnalysen via Desk Research als pragmatisch eingeordnet werden kann. $\mathrm{Zu}$ den bereits genannten Gründen für diesen Pragmatismus, zum einen die manuelle Durchführung ohne den Einsatz von Tools oder die Beauftragung einer Agentur und zum anderen die nicht standardisierte Umsetzung, kommt ein dritter Aspekt hinzu: Das Unternehmen setzt Social-Media-Analysen über Desk Research ohne methodische Systematisierung oder Reflexion um. Der offenbarte Pragmatismus ist nicht zuletzt auch darin begründet, dass die Praktiker keine fundierten Kenntnisse sozialwissenschaftlicher Forschungsmethoden besitzen und so die Methode auch nicht diesen entsprechend anwenden können. Wie gezeigt, nutzen die Befragten Social-Media-Analysen über Desk Research auf diverse Arten und in unterschiedlichem Kontext. Außerdem kombinieren sie diese methodische Umsetzung von Social-Media-Analysen mit weiteren, die sodann vorgestellt werden.

\subsubsection{Social-Media-Analysen über Tools}

Eine Möglichkeit, Social-Media-Analysen auf eine professionellere Art als über Desk Research durchzuführen, sehen die Befragten im Einsatz von Tools, eine andere Option erkennen sie in einer Beauftragung von Agenturen. Doch beide Varianten sind mitunter nur vermeintlich professionell. Der vorliegende Abschnitt thematisiert die Umsetzung von Social-Media-Analysen über Tools. ${ }^{6}$

Begründet ist die im Vergleich als standardisiert wahrgenommene Variante von Social-Media-Analysen via Tools darin, dass die Mehrheit dieser als einfach und immer auf die gleiche Art bedienbar dargestellt wird. Über die hinter den Tools stehende Methodik machen sich die Praktiker offenbar keine Gedanken. Einzelne Tools werden käuflich erworben und im Anschluss unmittelbar genutzt. Social-Media-Analysen mit Tools werden wie auch Desk Research unternehmensintern umgesetzt, von den Befragten selbst. Manche Social-Media-Analysten nutzen lediglich ein einzelnes Tool, mitunter auch, da nur ein Tool zur Verfügung steht, andere Praktiker können hingegen unterschiedliche Tools einsetzen, was parallel, aufeinander folgend oder selektiv, der jeweiligen Fragestellung entsprechend geschieht. Ob den praktischen Anwendern mehrere Tools zur Verfügung stehen oder diese nur über ein Tool verfügen, variiert erheblich zwischen den

\footnotetext{
${ }^{6}$ Aus forschungsethischen und rechtlichen Gründen nennt diese Arbeit die Namen der Tools nicht.
} 
Unternehmensbereichen, denen sie angehören. In den diversen Bereichen liegen den Untersuchungen ferner heterogene Fragestellungen zugrunde; die Einsatzgebiete von Social-Media-Analysen über Tools sind durchaus unterschiedlich. Wie bereits erwähnt, kombinieren die Social-Media-Analysten auch mehrere Varianten der Durchführung miteinander. Social-Media-Analysen werden zum Beispiel in einem ersten Schritt über Desk Research umgesetzt und anschließend um eine Durchführung über Tools ergänzt oder unternehmensintern mit Tools angewendet und zugleich um die Beauftragung eines Dienstleisters erweitert; mehrere Varianten der Kombination sind denkbar und kommen zur Anwendung. Insbesondere bei den professionelleren Arten der Durchführung ist es nicht ausgeschlossen, dass diese als automatisierte Formen auf in der Vergangenheit manuell als Desk Research realisierten und eher pragmatisch umgesetzten Social-Media-Analysen beruhen.

Eine Durchführung von Social-Media-Analysen über Tools beschreiben die Befragten als ,iterativen Prozess“. Dieser „,iterative Prozess“ findet zum Beispiel parallel zu manuell praktizierten Social-Media-Analysen über Desk Research beziehungsweise zur Umsetzung einer Agentur oder aber gleichzeitig in allen drei Varianten statt. Mit der Darstellung von Social-Media-Analysen als ,iterativen Prozess" meinen die praktischen Anwender, dass sie die Untersuchungen in Eigenregie Schritt für Schritt durchführen. Zunächst werde der Fragestellung offen begegnet, um sodann Themen, die für die Beantwortung der Fragestellung relevant scheinen, zu kategorisieren. Da erst nach einer ersten Durchsicht des für die Beantwortung der Fragestellung relevanten Datenmaterials eine Kategorisierung erfolgt, kann hier von einem explorativen Vorgehen bei Social-MediaAnalysen gesprochen werden. Einzelne Phänomene, welche über die Nutzung von Tools hervortreten, würden verschiedenen Themengebieten zugeordnet. Diese Vorgehensweise weist eine gewisse Ähnlichkeit mit zwei dem sozialwissenschaftlichen Methodenkanon entstammenden Verfahren auf. Einerseits erinnert die eben genannte Vorgehensweise bei der Durchführung von Social-Media-Analysen mit Tools an eine quantitative, andererseits an eine qualitative Inhaltsanalyse. Allem voran unterscheiden sich diese Verfahren zur Datenauswertung insofern voneinander, als dass sich eine quantitative Inhaltsanalyse dadurch auszeichnet, dass Themengebiete oder Kategorien, welche im Verlauf der Datenauswertung mit Inhalten gefüllt werden, bereits a priori in einem sogenannten „Codebuch“ festgelegt werden; demgegenüber ist eine qualitative Inhaltsanalyse dadurch gekennzeichnet, dass ihre Kategorien aus dem Datenmaterial heraus entstehen oder vielmehr entwickelt, erst im Verlauf der Datenauswertung mit Inhalt gefüllt und wiederholt modifiziert werden. Somit weist die qualitative Variante der Inhaltsanalyse wiederum eine Ähnlichkeit mit der auch in der vorliegenden Studie angewandten 
Grounded Theory Methodologie auf. Ob das Vorgehen bei Social-Media-Analysen über Tools, wie die Praktiker es beschreiben, eher einer quantitativen oder qualitativen Inhaltsanalyse entspricht, kann anhand des untersuchten Datenmaterials nicht eindeutig bestimmt werden; hier bleiben die Social-Media-Analysten zu vage. Dies bestätigt, dass bei dem Einsatz von Tools nicht von einer reflektierten und auf fundierten Kenntnissen der sozialwissenschaftlichen Forschungsmethoden beruhenden Auseinandersetzung mit der Methodik auszugehen ist. Es scheint vielmehr so, als würden diese unhinterfragt zur Anwendung gebracht werden. Jedoch ist, auch wenn die Befragten keine eindeutigen Angaben hierzu machen, anzunehmen, dass die einzelnen für über Tools umgesetzte Social-Media-Analysen relevanten Kategorien bereits a priori feststehen. Daher ist eine Nähe zu quantitativen Inhaltsanalysen zu vermuten. Begründet ist diese Annahme auch darin, dass die Kategorisierung von Themen bei Social-Media-Analysen mit Tools automatisiert erfolgt. Selbst wenn diese Tools auf ausgereiften Algorithmen beruhen, kann eine automatisierte Zuordnung zu Kategorien nur dann erfolgen, wenn diese im Vorfeld festgelegt wurden. Obwohl die Praktiker dieses für einen Vergleich mit sozialwissenschaftlichen Methoden nötige Detail nicht berichten, stellt dieser Abschnitt den skizierten, „,iterativ“ genannten Prozess beziehungsweise dessen Methodik noch etwas näher vor.

Laut den praktischen Anwendern werden in einem Schritt neben der zu untersuchenden Fragestellung die für diese relevanten und damit in die jeweilige Social-Media-Analyse mit einzubeziehenden Quellen festgelegt. Im Anschluss daran würden dann über die eingesetzten Tools entsprechende Daten aus sozialen Medien erhoben beziehungsweise Letztere einem Screening mithilfe der Tools unterzogen. Dies geschehe vollkommen automatisiert anhand von in den Tools angelegten Suchanfragen und deren individueller Ausstattung mit von den Befragten selbst formulierten und kombinierten Suchbegriffen oder auch Suchterminologien und Synonymen. An die Datenerhebung schließt deren Aufbereitung und Auswertung in Form der Kategorisierung in Themengebiete in Bezug auf die zugrunde liegende Fragestellung an; dies geschehe ähnlich einer quantitativen Inhaltsanalyse. Beispielhaft wird die Zuordnung erhobener Daten zu Produktthemen, Händlerthemen oder auch allgemein zum Themengebiet der Kommunikation über das eigene Unternehmen und dessen Marken angeführt. Die einzelnen Phasen der Erhebung, Aufbereitung und Auswertung der Daten werden mit deren Validierung in bis zu drei Schritten, die auf eine Verbesserung der Datenqualität zielt, abgeschlossen. Teilweise assoziieren die praktischen Anwender ihre Vorgehensweise bei Social-Media-Analysen über Tools mit einem Bastelprozess, vor allem bei ihrer Darstellung der Formulierung von Suchbegriffen sowie deren Sichtung und Ergänzung um Synonyme. Insgesamt wird für die Durchführung 
von Social-Media-Analysen mit Tools bis zum Erhalt der Ergebnisse circa eine Woche Zeit einkalkuliert. Dies ist jedoch mit der Einschränkung verbunden, dass bereits die erforderlichen Grundlagen für eine Durchführung von Social-MediaAnalysen mit Tools geschaffen sind; hierzu zählt zum Beispiel die Verfügbarkeit über ein Tool.

Alternativ werden Social-Media-Analysen auch ausschließlich über Tools durchgeführt. In den entsprechenden Fallbeispielen besteht gewissermaßen eine Ausschließlichkeit von Social-Media-Analysen über Tools, in dem Sinne, dass diese nicht mit anderen Varianten der Durchführung, wie etwa Desk Research oder einer Beauftragung von Dienstleistern, kombiniert werden. Die praktischen Anwender führen Social-Media-Analysen dann mit Tools unternehmensintern durch. Zum Zweck der eigenständigen sowie ausschließlichen Umsetzung von Social-Media-Analysen über Tools wurde beispielsweise nach einer Beratung durch Agentur (R) das Tool (5) gekauft. Unter Einsatz dieses Tools würden seitdem alle Social-Media-Analysen selbst umgesetzt. Im Rahmen der Beratung habe der Dienstleister auch Vorschläge für die Zusammenstellung von relevanten Schlüsselbegriffe, anhand derer das Tool soziale Medien durchsucht, gemacht und die Grundeinrichtung des Tools vorgenommen. Obwohl die Agentur zunächst quantitative Ergebnisse berichtet und Ergebniszusammenfassungen geliefert hat, sei es den Praktikern im Unternehmen von Beginn an möglich gewesen, das Tool selbst zu bedienen. Social-Media-Analysen über Tools beinhalten also quantitative und ,qualitative“ Anteile. Zum Beispiel werden Frequenzanalysen und Tonalitätsanalysen, die die praktischen Anwender selbst oft als „qualitative“ Elemente von Social-Media-Analysen bezeichnen, vorgenommen. Im Zusammenhang von Social-Media-Analysen mit Tools bedeuten Frequenzanalysen eine Ermittlung der Klickzahl auf den eigenen Webseiten und können damit auch als deren Überwachung verstanden werden. Die Befragten verstehen Tonalitätsanalysen, auch in dem hier beschriebenen Fallbeispiel, als ,qualitative" Analysen und beschreiben sie als eine Zusammenstellung relevanter Schlüsselbegriffe. Werden Social-Media-Analysen ausschließlich mit Tools praktiziert, finden sich neben Frequenzanalysen und Tonalitätsanalysen zudem Inhaltsanalysen, Videoanalysen und Influencer-Analysen. Inhaltsanalysen bedeuten, dass die Praktiker untersuchen, welche Social-Media-Kanäle von Bedeutung sind. Mit Videoanalysen messen sie, ähnlich wie bei Frequenzanalysen, Reichweiten über die Anzahl der „Views“, also der Klickzahl, die ein bereitgestelltes Video erzielt hat. Über von den Befragten als solche bezeichnete „Influencer-Analysen“ werden ferner ,Einflussgrößen“ sozialer Medien im Sinne von Meinungsführern ermittelt; auch dies geschieht anhand von quantitativen Ergebnissen in Form deren Reichweiten. 
Genaue Beschreibungen der den verschiedenen Analysevarianten zugrunde liegenden Methodik sind den Ausführungen der Social-Media-Analysten nicht zu entnehmen. Dies ist ein Indiz dafür, dass auch Social-Media-Analysen mit Tools von den Befragten ohne fundierte Kenntnisse sozialwissenschaftlicher Forschungsmethoden umgesetzt werden. Auch für diese Art von Social-MediaAnalysen wählen die Praktiker Bezeichnungen sozialwissenschaftlicher Verfahren, wie „Inhaltsanalysen“ und „Frequenzanalysen“. Bei den Befragten scheint kein Bewusstsein dafür vorhanden zu sein, dass es sich dabei um etablierte Termini der Sozialforschung handelt. Sie praktizieren Social-Media-Analysen über Tools offenbar methodisch unreflektiert. Durchgeführt werden diese in der Regel in feststehenden Rhythmen, beispielsweise jährlich für eine „Beobachtung“ von für die Automobilindustrie relevanten Messen und Veranstaltungen.

Mit dem Ziel der vollständigen Automatisierung von Social-Media-Analysen mit Tools ist ein Unternehmensbereich des betrachteten Automobilherstellers eine Kooperation mit einem Toolanbieter eingegangen. Auch diese Zusammenarbeit zur Optimierung eines bestimmten Tools stellt eine weitere Variante, wenn auch eine Sonderform, der Umsetzung von Social-Media-Analysen über Tools dar. Begründet ist dieser Weg laut den Praktikern im IT-Hintergrund ihrer Abteilung und der Erkenntnis, dass manuelle Untersuchungen wegen der großen, in sozialen Medien vorhandenen Datenmenge nicht funktionieren. So sei Social-Media-Analysen mit technischem Fokus und Text-Mining-Algorithmen begegnet worden, um deren Durchführung komplett zu automatisieren. Im Vorfeld von Social-Media-Analysen gelte es Fragestellungen zu definieren, die über in Automobilforen eingesetzte Text-Mining-Verfahren beantwortet werden:

\footnotetext{
Befragter: [... ] [A]lso (stockt) die grundlegende Prämisse war, also das, was wir hier tun, muss dann, weitestgehend automatisiert funktionieren, ja. Also ich definiere ein bestimmtes Fragen-Set, ja, dann mit diesem Fragen-Set mache ich eben mein TextMining in, ja, hauptsächlich dann Automotive-Foren, wo ich es dann letzten Endes fahre, weil bei Facebook und Twitter da relativ wenig reinkam, konnte ich dann eben über diese Automotive-Foren, über diese Beiträge, die von uns gestellten Fragestellungen auch wirklich beantworten auf (stockt) einer weitestgehend automatisierten Art und Weise (I25/ S4/ Z34-40).
}

Im untersuchten Datenmaterial ist das Vorgehen mit dem Toolanbieter dargestellt. Zunächst sei ein „Proof of Concept“ erfolgt, also die prinzipielle Durchführbarkeit des Projekts auf Basis der Technologie geprüft worden. Es seien zwar auch andere Anbieter gesichtet worden, doch es habe sich bestätigt, dass der ausgewählte Toolanbieter die fortschrittlichste Technologie offeriere. Alternative Tools 
würden ein Baukastenformat aufweisen und lediglich statistische Standardabfragen über Dashboards ermöglichen. So könnten bei den anderen Anbietern und deren Tools die Social-Media-Analysten zwar Suchanfragen von bis zu circa 20 Zeilen formulieren und Auswertungen zu bestimmten Stichwörtern vornehmen, doch derartige Ansätze seien für die relevanten Themen nicht geeignet beziehungsweise wären veraltet. So sei die Kooperation mit dem ausgewählten Anbieter eingegangen und mit dieser das Ziel der vollständigen Automatisierung von Social-Media-Analysen über Verfahren des Text Mining und Machine Learning oder der Mustererkennung allgemein verfolgt worden. Aus dem untersuchten Datenmaterial geht hervor, dass hinter diesem Vorgehen der Anspruch steht, die bei der Umsetzung von Social-Media-Analysen angewandten Suchanfragen künstlich intelligent, also selbstlernend umzusetzen und damit beständig anzupassen. Auch die Zusammenarbeit mit dem Toolanbieter beschreiben die Befragten als ,iteratives“ und pragmatisches Vorgehen. Vor allem habe es im gemeinsamen Trainieren von Algorithmen bestanden, wobei der Toolanbieter über Crawler oder Software-Filter des Weiteren Aussagen aus Automobilforen über Extraktion ermittelt und zur weiteren Auswertung aufbereitet hat. Sie haben dann, so führen die Befragten weiter aus, einzelne Aussagen ausgewertet. Aufgrund seines IT-Hintergrunds habe ihr Unternehmensbereich diverse Textanalysen und Sentiment-Analysen selbst durchgeführt und zu diesem Zweck sein gesamtes Wissen zu Advanced Analytics, Machine Learning und Big Data genutzt. Letztlich habe die Kooperation mit dem Toolanbieter darin bestanden, dass dessen Crawler für die Erhebung von Daten aus sozialen Medien genutzt wurden. Die Daten wurden dann eigenständig analysiert. Das Ziel sei es gewesen, das Tool durch Künstliche Intelligenz zur einer automatisierten Mustererkennung zu befähigen, um verlässliche Vorhersagen treffen zu können. Die befragten Praktiker nennen dies „Predictive Analytics“ und assoziieren ihre Vorgehensweise mit dem „Lesen einer Kristallkugel“ und die dadurch gewonnenen Ergebnisse mit dem „heiligen Gral“, den es zu finden gilt. Um dieses langfristige Ziel zu erreichen, müsse jedoch, so ergänzen sie, die Unternehmenskultur und auch das in ihrem Unternehmen vorherrschende ,Mindset" unbedingt modifiziert werden.

Eine weitere Möglichkeit der Nutzung von Social-Media-Analysen mit Tools besteht darin, dass diese zusätzlich, also parallel zur Durchführung durch Agenturen eingesetzt werden. Fallberichte zeigen, dass zwar Dienstleiser SocialMedia-Analysen umsetzen, das Unternehmen parallel dazu aber weitere SocialMedia-Analysen selbstständig über Tools durchführt. Es werden mehrere Tools genannt, die hierfür geeignet sind. Über ihren Einsatz werde untersucht, auf welchen Social-Media-Kanälen welche Themen auf welche Art besprochen werden. Ferner werde die eigene Leistung gemessen; durch die Überwachung feststehender KPIs würden die eigenen Aktivitäten in sozialen Medien kontrolliert. So 
würde ermittelt, ob die veröffentlichten Inhalte und Zeitpunkte der Bereitstellung funktionieren. Bei dieser Art der Umsetzung von Social-Media-Analysen stehen quantitative Ergebnisse im Fokus. Die über Social-Media-Analysen mit Tools generierten Ergebnisse dienen der Überwachung des eigenen Handelns.

Es bleibt festzuhalten, dass Social-Media-Analysen mit Tools von den Praktikern selbst aus dem Unternehmen heraus durchgeführt und als professionellere, da gewissermaßen standardisierte Variante der Methode eingeordnet werden. Dies hängt mitunter damit zusammen, dass die Mehrheit der Tools als einfach und immer auf die gleiche Weise bedienbar dargestellt wird. Teilweise nutzen die Befragten lediglich ein Tool, teilweise kommen mehrere Tools zum Einsatz. Die Umsetzung von Social-Media-Analysen über Tools findet entweder ausschließlich oder in Kombination mit anderen Varianten der Durchführung von Social-MediaAnalysen statt. Die den Untersuchungen zugrunde liegenden Fragestellungen können ebenso variieren wie die Einsatzgebiete der Methode. Gemeinhin wird die Umsetzung von Social-Media-Analysen über Tools als „iterativer Prozess“ beschrieben, der als „offenes“, exploratives Verfahren beginnen kann. SocialMedia-Analysen, die über Tools umgesetzt werden, erinnern in ihrem Verlauf dann sowohl an quantitative als auch an qualitative Inhaltsanalysen, wobei aufgrund zu vager Berichte der praktischen Anwender keine eindeutige Zuordnung zu einer der beiden sozialwissenschaftlichen Methoden möglich ist; aufgrund der Algorithmen, auf welchen Tools beruhen, wird eine Nähe zu quantitativen Inhaltsanalyse angenommen. Mit Tools setzen die befragten Praktiker Frequenzanalysen, Tonalitätsanalysen, Inhaltsanalysen, Videoanalysen und Influencer-Analysen um. Ebenso arbeiten sie an der Befähigung zum „Lesen einer Kristallkugel“ und zum Aufspüren des ,heiligen Grals“ und meinen die vollständige Automatisierung von Social-Media-Analysen mit Tools als „Predictive Analytics“. Allerdings erwecken die Befragten insgesamt den Anschein, als würden sie sich kaum Gedanken über die hinter den Tools stehende Methodik machen.

\subsubsection{Social-Media-Analysen mit Agenturen}

Von den in den letzten beiden Abschnitten dargestellten Arten des Vorgehens bei Social-Media-Analysen ist eine weitere zu unterscheiden. Es handelt sich dabei um die Durchführung von Social-Media-Analysen mit Agenturen oder eine Beauftragung von Dienstleistern zur vollständigen Umsetzung von Social-MediaAnalysen, an deren Ende dann die Lieferung der Ergebnisse an das Unternehmen steht. ${ }^{7}$ Die Umsetzung von Monitoring und Listening durch Agenturen wird wie die über Tools von den Befragten als professionellere Art dargestellt.

\footnotetext{
${ }^{7}$ Aus forschungsethischen und rechtlichen Gründen werden die Namen der Agenturen in dieser Studie ebenso wenig genannt wie die Namen der Tools.
} 
Allerdings ist auch diese Durchführungsweise mitunter nur vermeintlich professionell. Die Praktiker nehmen die Umsetzung durch einen Dienstleister als vergleichsweise standardisiert wahr, wobei die Standardisierung zumeist mit einer Professionalisierung assoziiert oder sogar gleichgesetzt wird.

Der Ursprung einer Beauftragung externer, Monitoring und Listening als Dienstleistung anbietender Agenturen kann zwischen diversen Befragten und Unternehmensbereichen durchaus variieren. Zum einen berichten Praktiker, dass das Thema "Social-Media-Analysen“ von Beginn an gemeinsam mit Dienstleistern behandelt wurde; zum anderen finden sich Ausführungen, aus denen hervorgeht, dass Social-Media-Analysen zunächst unternehmensintern von Mitarbeitern praktiziert wurden, über Desk Research oder mit Tools, und aus verschiedenen Gründen dann an Agenturen ausgelagert wurden. Ferner werden Monitoring und Listening in einigen Fällen parallel sowohl von den Befragten im Unternehmen via Desk Research oder Tools umgesetzt als auch von Dienstleistern. Dabei handelt es sich dann entweder um eine inhaltliche „Detaillierung“" oder eine thematische Erweiterung oder aber auch um eine Kontrolle der Arbeit der Dienstleister. Social-Media-Analysen mit Agenturen markieren somit eine weitere Option im ,Sammelsurium“ der Varianten der Methode, die dem deutschen Automobilhersteller zur Verfügung stehen. Unterschiedliche Social-Media-Analysen werden mitunter auch parallel durchgeführt. Je nach Unternehmensbereich, der eine Untersuchung umsetzt, und der Fragestellung, die dieser zugrunde liegt, werden Social-Media-Analysen mit Agenturen als einzige Variante der Methode praktiziert oder mit alternativen Optionen kombiniert. Fragestellungen, denen das Unternehmen mit von Agenturen umgesetztem Monitoring oder Listening nachgeht, divergieren und sind zu einem späteren Zeitpunkt noch einmal Thema.

Sofern Monitoring und Listening in erster Linie oder ausschließlich über Dienstleister umgesetzt werden, erfolgt dies oftmals über einen Rahmenvertrag, der zwischen einer Agentur und dem deutschen Automobilhersteller besteht. Das Datenmaterial zeigt, dass der deutsche Automobilhersteller bislang vor allem drei Agenturen, die die Praktiker namentlich nennen, mit der Durchführung von Social-Media-Analysen beauftragt hat. Jedoch verfügen die Befragten nicht nur über Erfahrungen mit diesen Dienstleistern, sondern haben teilweise bereits weitere kennengelernt. Manche praktischen Anwender können auf Erfahrungen mit mehreren Agenturen zurückgreifen, andere haben bisher nur mit einem Dienstleister gearbeitet. Letzteres bedeutet nicht selten, dass diesem, der wegen eines Rahmenvertrags oftmals seit Langem für einen Unternehmensbereich tätig ist, fast uneingeschränkt, gewissermaßen ,blind vertraut' wird. Die Arbeit einer Agentur wird in solch einem Fall kaum angezweifelt und hinterfragt oder mit der von anderen Agenturen verglichen. Auf Seiten der Befragten sind zumeist nicht 
vorhandene oder fundierte Kenntnisse des sozialwissenschaftlichen Methodenkanons und insbesondere auch Unwissen in Bezug auf die hinter Monitoring und Listening stehende Methodik beziehungsweise bezüglich sozialer Medien im Allgemeinen Ursache für dieses nahezu ,blinde Vertrauen' in Agenturen. Allerdings bedingen sich dieses Unwissen und fast ,blinde Vertrauen' wiederum gegenseitig. Im Rahmen der Datenauswertung dieser Arbeit ist in derartigen Fällen, die häufig im untersuchten Datenmaterial vorzufinden sind, mit dem Begriff „Agenturvertrauen“ gearbeitet worden.

Ferner ist an dieser Stelle vorauszuschicken, dass einige Befragte, die von kooperativ mit Agenturen durchgeführten Social-Media-Analysen berichten, anmerken, dass sie selbst keine Erfahrung in der Umsetzung von Monitoring und Listening haben. Mit diesem Hinweis meinen die Praktiker, dass sie Social-Media-Analysen nicht selbst durchgeführt, sondern diese nur bei einem Dienstleister in Auftrag gegeben haben. Sie waren folglich bei der Durchführung der Untersuchungen durch die Agentur selbst nicht anwesend. Für manche Befragte ist dies Anlass zu der Annahme, dass sie selbst nicht über Erfahrung mit Social-Media-Analysen verfügen. Im Rahmen dieser Studie stellen jedoch gerade diese Erfahrungen eine von mehreren Varianten der angewandten Praxis von Social-Media-Analysen dar, wie sie in dem betrachteten Unternehmen vorzufinden ist, und sind gerade als solche von Bedeutung. Vielmehr noch beabsichtigen die Social-Media-Analysten mit derartigen Hinweisen, Erwartungen, welche sie selbst bei der sie interviewenden Forscherin vermuten, zu mindern und ihre eigenen Berichte bezüglich der Umsetzung von Social-Media-Analysen zu nivellieren. Ausführungen der befragten Praktiker mit derartigen Anmerkungen sind aber dennoch oder gerade deshalb in die Datenauswertung und diesen Ergebnisbericht eingeflossen.

Wenn Social-Media-Analysen von Dienstleistern durchgeführt werden, geschieht dies in Gänze oder zumindest die Datenerhebung über einen Einsatz von Software, Tools oder Crawlern. Im Hinblick auf die Auswertung der aus sozialen Medien erhobenen Daten unterscheiden sich einzelne Anbieter allerdings erheblich voneinander. Entweder erfolgt auch die Datenauswertung automatisiert, über den Einsatz spezieller Software oder Tools, oder aber manuell in Form einer Codierung durch menschliche Analysten, was dann zumeist als ,,intellektuelle“ Recherche oder Auswertung bezeichnet wird. Einige SocialMedia-Analysten haben über ihre Praktiken berichtet, die wegen ihres singulären Auftretens im vorliegenden Datenmaterial als Sonderformen einzustufen sind. Dabei handelt es sich um Umsetzungen mit weiteren, bislang in dieser Arbeit nicht angesprochenen Agenturen, deren Kompetenz nicht nur im Bereich von 
Social-Media-Analysen liegt, die sie aber trotzdem anbieten, und um eine Kooperation mit der Wissenschaft zur Erprobung ihres Angebots. Im Folgenden wird zunächst ein Überblick über die „Sonderformen“ genannten Praktiken von SocialMedia-Analysen gegeben, um im Anschluss daran gesondert auf deren Umsetzung von den am häufigsten genannten Dienstleistern einzugehen; dies erneut in Gestalt einer deskriptiven Zusammenfassung.

\subsection{Social-Media-Analysen mit Agenturen als Sonderformen}

Befragte haben von Social-Media-Analysen mit Agenturen als Sonderformen berichtet. Ihre Erfahrungen werden als „Sonderformen“ bezeichnet, da sie sich mit Blick auf das gesamte Datenmaterial durch ihr singuläres Auftreten von der gängigen Praxis mit Dienstleistern abheben. So haben zwar nur vereinzelte Social-Media-Analysten von diesen Praktiken berichtet, doch erst deren Erfahrungen vervollständigen die gesamte Darstellung von Social-Media-Analysen mit Dienstleistern. Zunächst geht es um einen Erstkontakt mit einer Agentur, in einem nächsten Schritt um die Kooperation mit einer wissenschaftlichen Forschungseinrichtung und zuletzt um die Zusammenarbeit mit einem Dienstleister, der zwar nur einmal beauftragt wurde, zu dessen Beauftragung aber ein Bericht vorliegt, der in Bezug auf die darin enthaltene Thematisierung methodischer Aspekte heraussticht.

Im Hinblick auf eine Durchführung von Social-Media-Analysen mit Dienstleistern hat ein Befragter berichtet, dass er selbst bisher zwar noch keine Social-Media-Analysen umgesetzt hat, dies bezüglich aber bereits von drei Agenturen, (S), (T) und (P), beraten wurde. Damit hat der Befragte zwar keine praktische Erfahrung in der Umsetzung von Monitoring und Listening, ist den ersten Schritt dieser Variante der Durchführung, der in einer Beratung durch die Dienstleister besteht, aber bereits gegangen. Im Fokus der Beratung hätten methodische Aspekte gestanden; es sei um die Frage der Eignung der Methode für Medienresonanzanalysen und um die Gegenüberstellung der quantitativen und ,qualitativen“ Datenauswertung gegangen. Die Intention zur Durchführung von Social-Media-Analysen mit Agenturen habe darin bestanden mediale Kommunikation zu quantifizieren. Aus dem Austausch mit dem Dienstleister sei hervorgegangen, dass bei Social-Media-Analysen über automatisierte Algorithmen zunächst Häufigkeiten bestimmt und diese im Anschluss nach Tonalität geclustert würden. Alternativ gebe es die Option einer manuellen Codierung, doch eine solche würden nur ,hochwertige“ Agenturen anbieten: 
Befragter: Mhm (überlegt). Ja. Also es gibt ja unterschiedliche, äh, Verfahren. Einmal/ also die großen Unterschieden sind eigentlich, das Eine ist, äh, du zählst die Kommentare, äh, und clusters die quasi nach einem automatischen Algorithmus in gut oder in schlecht oder in neutral. Und, ähm, dann gibt es halt etwas, äh, hochwertigere Vercodungs-Anbieter, wie beispielsweise auch das Instrument, das uns [Agentur S] vorgestellt hat, die natürlich dann teurer sind, wo sich dann aber die Leute auch hinsetzen und halt einen gewissen Anteil der Nennungen eben auch wirklich manuell zuordnen. Was halt eine höhere Genauigkeit, äh, ist. Und, gerade bei der Sprache, die auch im Netz, äh, verwendet wird, ist, äh, ist das, glaube ich, ähm, auch ein, ja, notwendig/, äh, notwendiges Thema. Weil, wenn du zum Beispiel irgendwie so einen Kommentar hast und das/ das ist ja/ ist ja durchaus, äh, gegeben, ,Mann, ist der scheiße-geil!““.

Interviewer: Mhm (bejahend) (Lachen).

Befragter: (Lachen). Mhm. Ist das jetzt positiv (Lachen) oder ist das jetzt negativ? Und ein automatisches/, äh, eine automatische Systematisierung wird sich da wahrscheinlich schwer tun (I14/ S4/ Z17-31).

Diese Aussage zeigt, dass bei Social-Media-Analysen mit Dienstleistern die Art der Datencodierung als der wesentliche Unterschied zwischen einer automatischen und manuellen Auswertung angesehen wird. Erstere verstehen die meisten Befragten dabei als quantitative, letztere als ,qualitative' Datenauswertung.

Ein anderer Befragter beschreibt eine weitere Sonderform von Social-MediaAnalysen. Es handelt sich um eine Kooperation mit einer Forschungseinrichtung zur Erprobung deren selbstentwickelter Art von Social-Media-Analysen. Da der Automobilhersteller für die Umsetzung bezahlt hat, wird die Forschungseinrichtung in dieser Arbeit ebenfalls als Dienstleister eingeordnet. Einerseits sei mit der Social-Media-Analyse das Ziel verfolgt worden, Antworten auf die zugrunde gelegte Fragestellung zu erhalten, andererseits sei die Methode selbst sowie ihre Akzeptanz und Durchführbarkeit im Unternehmen getestet worden. In Bezug auf die methodische Umsetzung und praktische Durchführung sei eine Abstimmung mit der Agentur über circa drei Videokonferenzen erfolgt. Gemeinsam sei definiert worden, welche Foren inhaltlich untersucht werden sollen. Ein Professor hätte mit seinen Studenten die Social-Media-Analyse automatisiert mithilfe eines Algorithmus durchgeführt, Daten in sozialen Medien über einen Zeitraum von circa sechs Wochen erhoben und dann einer "Schwarmanalyse“ unterzogen. Es liegt die Vermutung nahe, dass „Schwarmanalysen“ und Influencer-Analysen einander entsprechen und ebenfalls rein automatisiert durchgeführt werden; dies kann mangels weiterer Erklärungen des Befragten jedoch nicht bestätigt werden. Weiterhin hat der Befragte berichtet, dass mit dieser Social-Media-Analyse die aktuelle Wahrnehmung in sozialen Medien zu zwei Produkten während ihrer Markteinführung untersucht wurde. In diesem Zusammenhang seien Frequenzanalysen und Tonalitätsanalysen zum Einsatz gekommen, um zu ermitteln, wie 
häufig eines der beiden Produkte in den im Vorfeld festgesetzten sozialen Medien erwähnt wird und wie die Erwähnungen konnotiert sind. Die Forschungseinrichtung hat die Methode als „Cool Hunting“ bezeichnet. Aufgrund der Darstellung dieser Methode durch den Befragten ist davon auszugehen, dass die Durchführung einer vollständig automatisierten Social-Media-Analyse entspricht. InfluencerAnalysen, die durchgeführt wurden, beruhen letztlich auch auf analysierten Frequenzen. Ziel sei es, Erfolgsfaktoren von neu in einen Markt eingeführten Produkten zu messen und zu ermitteln, auf welchen Faktoren der Erfolg dieser beruht. Während der Durchführung des Projekts sei dieses dokumentiert worden; zum Abschluss habe das Unternehmen einen Ergebnisbericht erhalten. Es bleibt bezüglich dieser, hier als Sonderform eigestufte Social-Media-Analyse festzuhalten, dass zu deren Methodik nur wenig berichtet wurde. Daher kann diese nicht näher dargestellt oder gar beurteilt werden. Allerdings liegt die Vermutung nahe, dass die gesamte Durchführung automatisiert erfolgt ist und die eigentliche Umsetzung kaum Unterschiede mit einer Durchführung von Monitoring und Listening durch andere Agenturen aufweist. Zu dem Befragten ist zu ergänzen, dass dieser weder Kenntnisse sozialwissenschaftlicher Forschungsmethoden noch Interesse an der hinter der Social-Media-Analyse stehenden Methodik zu haben scheint. Daher kommt auch hier der Code „Agenturvertrauen“ zum Tragen. Er beschreibt in diesem Fall, dass die Social-Media-Analyse bei einer Agentur beauftragt und nach einem Erstgespräch nicht mehr reflektiert wurde. Der Bericht des Befragten macht deutlich: Nicht nur Agenturen können mit Monitoring und Listening beauftragt werden, sondern auch wissenschaftliche Forschungseinrichtungen, und dies über nationale Grenzen hinweg.

Eine dritte Sonderform von Social-Media-Analysen markiert deren Durchführung mit Agentur (Q), von der ein praktischer Anwender berichtet. Zwar enthält das untersuchte Datenmaterial Ausführungen über Kooperationen mit insgesamt drei Agenturen, $(\mathrm{Q}),(\mathrm{O})$ und $(\mathrm{N})$, die nicht zugleich eine derjenigen drei sind, mit denen bereits mehrere Social-Media-Analysen umgesetzt wurden und die noch gesondert vorgestellt werden, doch soll von diesen drei in diesem Abschnitt nur Agentur (Q) näher betrachtet werden. Bezüglich der anderen beiden schildern die Praktiker keine Inhalte, welche eine nähere Beleuchtung rechtfertigen würden. Der Bericht über die mit Agentur (Q) umgesetzte Social-Media-Analyse sticht deutlich hervor, da dieser die praktische Umsetzung und methodische Durchführung explizit behandelt. Einerseits ist dies wohl darin begründet, dass der befragte Praktiker Kenntnisse sozialwissenschaftlicher Forschungsmethoden zu besitzen scheint; andererseits lässt sich erahnen, dass auch die Arbeit des Dienstleisters auf solchen beruht. So vergleicht der Befragte die von ihm begleitete Social-Media-Analyse mit der Arbeit eines Ethnographen: 
Befragter: [... ] Das heißt also allgemeine Erfahrungen (stockt) mit Social Media, ähm, die Geschwindigkeit, (stockt) die hilfreich war, (stockt) die/ dieses sehr Ungefilterte und hier haben wir aber auch ein/ einen Zeitverlauf oder, ich habe den Eindruck, wie jetzt/ über die Zeit hat es sich verändert, weil/ als die Kunden 2009 angefangen haben, sich so zu vernetzen, hatte ich den Eindruck, haben sie sich auch wirklich völlig unbeobachtet gefühlt. Und das war halt für uns so ein bisschen die Perspektive, wirklich wie so ein (Lachen)/ wie so ein/ wie so ein Ethnograph im besten Sinne, (stockt) der gar nicht wahrgenommen wird (I32/ S7/ Z21-27).

Die Assoziation von Monitoring und Listening mit ethnographischen Methoden, die der Befragte herstellt, beruht auf dessen Annahme, dass in Social Media vorhandene oder darin erhobene Daten unter Ausschluss des Phänomens der ,,sozialen Erwünschtheit “ entstehen und daher ideal geeignet sind für die Untersuchung mit einem beobachtenden Verfahren als das er Social-Media-Analysen charakterisiert. Bevor für ein spezifisches Projekt des Unternehmensbereichs F\&E Social-MediaAnalysen in Zusammenarbeit mit einer bestimmten Agentur, die schon für die Nutzerforschung des Projekts beauftragt war, umgesetzt wurden, hätten Praktikanten diese intern im Unternehmen manuell mit Desk Research umgesetzt. Hinter der Übergabe der unternehmensinternen Umsetzung an die Agentur habe sich, so erläutert der Befragte, das Ansinnen verborgen, Social-Media-Analysen zu systematisieren. Ursprünglich sei mit der Methode wegen der zufälligen Entdeckung einer FacebookGruppe, in der ein Austausch über das Projekt stattgefunden hat, begonnen worden. Zunächst sei diese Facebook-Gruppe manuell, über Desk Research ,,beobachtet“ worden, nach Übernahme des Themas hätte der Dienstleister das Internet systematisch nach Diskussionen auf Social-Media-Kanälen durchsucht. Aufgrund einer eingeschränkten, für das Projekt relevanten Zielgruppe und damit einer ebensolchen Nutzergruppe sozialer Medien sind, so vermutet der Befragte, sämtliche relevante Social-Media-Plattformen, allen voran Facebook, sowie ein paar Foren und Blogs ausfindig gemacht worden, so dass von einer Vollerhebung aller zu dem Thema in sozialen Medien vorhandenen Daten ausgegangen wurde. Von Beginn an, so betont der Befragte, war das Vorgehen bei den Social-Media-Analysen rein ,qualitativ“ und manuell; später auch das der Agentur, das ,,redaktionell“ genannt wird. Ferner betont er, dass über die gesamte Projektdauer keine quantitativ orientierten Tools eingesetzt wurden, und begründet dies damit, dass diese stärker in die Breite gehen, was im Kontext des Projekts nicht notwendig war. Nach ihrer Erhebung seien die Daten im Hinblick darauf, worüber in sozialen Medien unter Bezugnahme auf das Projekt ein Austausch stattfindet, ausgewertet worden. Aus dem Datenmaterial geht hervor, dass der Unternehmensbereich zunächst Frequenzanalysen durchgeführt hat. Damit wurde untersucht, wie viele Kommentare in Social Media zu gewissen Themen zu verzeichnen waren und wie intensiv deren Diskussion im Vergleich mit anderen 
Themen war. Die gesamte Datenauswertung sei ausschließlich unter Einsatz ,qualitativer' Methoden erfolgt, anhand derer einzelne Daten letztlich in Themen oder Kategorien strukturiert wurden. Als Beispiele nennt der Befragte die Gegenstände der Diskussion oder offene Fragen und Lösungen dazu. Diese Kategorisierung der Daten durch Tonalitätsanalysen hätte einen Überblick über relevante, in sozialen Medien diskutierte Themen geliefert, deren Entwicklungen im Zeitverlauf „,beobachtet" wurde. In wöchentlichem Rhythmus und zum Abschluss jeder Untersuchung habe der Dienstleister dem Unternehmen einen objektiven Ergebnisbericht erstellt. Der Befragte weist darauf hin, dass ein solcher auch mit in sozialen Medien veröffentlichtem Bildmaterial und Originalkommentaren aus diesen angereichert war. Des Weiteren sei dem Bericht die Anzahl der untersuchten sozialen Medien sowie die der Nutzer, die sich in diesen zu relevanten Themen geäußert haben, zu entnehmen gewesen. Unternehmensintern seien die Ergebnisse der Social-Media-Analysen dann über einen entsprechenden Verteiler verbreitet worden. Zudem hat die Agentur, so ergänzt der Befragte, eine Datenbank aufgebaut, in der die Social-Media-Analysen und das in diesen untersuchte Datenmaterial archiviert wurden. Damit habe der Dienstleister vorhandene Ergebnisberichte und Daten in eine durchsuchbare Umgebung überführt und es möglich gemacht, schon untersuchte Daten und die daraus hervorgegangenen Ergebnisse auch im Nachhinein noch durchsuchen zu können. Die Schilderungen des Befragten bezüglich der praktischen Umsetzung oder methodischen Durchführung der Social-Media-Analysen erinnern an qualitative Inhaltsanalysen, vor allem wenn von der Kategorisierung der Daten in Themengebiete durch „qualitative" Methoden die Rede ist. Die vergleichsweise detaillierte Darstellung der hinter Social-MediaAnalysen stehenden Methodik zeigt, dass sich der Social-Media-Analyst damit auseinandergesetzt hat. Er scheint mit sozialwissenschaftlichen Forschungsmethoden vertraut. Die Social-Media-Analysen von Agentur (Q) stellen eine Sonderform in dieser Arbeit dar und dies in dreifacher Hinsicht: Erstens durch ihr singuläres Auftreten im Untersuchungsmaterial; zweitens aufgrund des Verzichts auf Tools und Software; drittens wegen der Charakterisierung als beobachtendes Verfahren und ethnographische Methode. Anhand dieser Aspekte unterscheiden sich die von Agentur (Q) umgesetzten Social-Media-Analysen deutlich von den mit anderen Dienstleistern durchgeführten.

\subsection{Social-Media-Analysen mit Agentur (X)}

Die Mehrheit der Befragten hat bereits eigene Erfahrungen in der Umsetzung von Social-Media-Analysen mit Agenturen gesammelt und ist über den ersten Schritt, der in einem Beratungsgespräch besteht, hinausgekommen. Wie Social-Media-Analysen mit Agentur $(X)$ praktisch umgesetzt und methodisch durchgeführt werden, behandelt der vorliegende Abschnitt. Dem Datenmaterial ist zu entnehmen, dass in den letzten Jahren zahlreiche Social-Media-Analysten Erfahrungen mit dieser Agentur 
gesammelt haben. Bezüglich der Anwendung der Methode mit Agentur (X) haben sie sowohl gemeinsame als auch verschiedene Erfahrungen geschildert. Der betrachtete Automobilhersteller hat mit Agentur (X) zwei Rahmenverträge für Monitoring und Listening geschlossen. Für diese Verträge sind mehrere Unternehmensbereiche freigeschaltet, so dass diese ohne Administrationsaufwand eine Durchführung einzelner Social-Media-Analysen abrufen können. Alle bei Agentur (X) bisher beauftragten Social-Media-Analysen wurden über diese Rahmenverträge durchgeführt.

Von Agentur (X) umgesetzte Social-Media-Analysen beschreiben die Praktiker, die von einer Kooperation mit diesem Dienstleister berichten, als „Recherchen im Internet“ oder „Recherchen in Foren“ und nennen diese „Netnographie“. Im Vorfeld der Umsetzung würde eine Abstimmung mit der Agentur erfolgen, um das Thema, zu untersuchende Produkte, Zeiträume, Sprachen oder Märkte sowie auch Foren, in denen Daten erhoben werden, festzusetzen und um die Erwartungen des Unternehmens an die Social-Media-Analyse zu klären. Da der Preis einer Social-Media-Analyse auch von der Anzahl der aus sozialen Medien erhobenen Daten abhängt, die letztlich ausgewertet werden, werde diese anhand des für die Social-Media-Analyse eingeplanten Budgets ebenfalls vorab festgelegt. Laut den Praktikern führt Agentur (X) Social-Media-Analysen zu diversen Themen, im Hinblick auf verschiedene Produkte und unterschiedliche Zeiträume durch. Außerdem könnten die Social-Media-Analysen für mehrere Sprachen und damit Märkte sowie in Bezug auf zahlreiche Foren sozialer Medien erfolgen. Begriffe, nach denen Agentur (X) sucht, würden auch im Vorfeld definiert:

Befragter: [... ] Haben vorher das Thema mit denen hier besprochen, erklärt, was wir so wissen wollen. Dann haben die oder wir gemeinsam dann so Vorschläge gemacht, das kannst du nicht/, ja, (flüstert) du kennst dich ja besser aus, vollautomatisieren, sondern, äh, du musst natürlich vorher schon ein bisschen wissen, was du wissen willst, und es dementsprechend aufsetzen. Und, ähm, dann überlegt, in welche Medien gehen wir da rein, in welche Länder gehen wir da rein? Macht natürlich dann auch alleine von der Umsetzung einen Riesen-Aufwand, ähm/, du musst das in mehreren Sprachen kennen, wie auch immer dort. Ähm, (...) platt gesprochen, nach welchen Schlagwörtern oder in welche Richtung/, was wollen wir denn erfahren? Worüber wollen wir was wissen? Und dann hat der quasi einen Vorschlag gemacht, wie man das dann, ähm, aufbaut. Und hat dann seinen Crawler losgeschickt und, es ist schon zu lange her, um mich an alles zu erinnern. Äh, ist aber auch noch, immer noch natürlich viel Handarbeit mit dabei. Ich glaube, wir sind trotzdem JEDEN Tweet oder Feed oder wie auch immer man das dann übergreifend nennt, äh, durchgegangen. Ähm, obwohl die schon irgendwie so eine Sentiment-Analyse, eben, ist das jetzt positiv, negativ, und was liest man dabei raus? Und die haben dann da auch irgendwie ein Portal, eine Webseite, wo man dann so Analysen fahren kann dazu (I15/ S1/ Z19-34). 
Das Untersuchungsmaterial zeigt, dass die Daten für die von Agentur (X) umgesetzten Social-Media-Analysen vorrangig in Automobilforen, automatisiert durch Einsatz eines von dem Dienstleister entwickelten Crawlers erhoben werden. Die erhobenen Daten würden anschließend sowohl einer quantitativen, durch eine Software automatisierten Untersuchung bezüglich der Ermittlung von Frequenzen, als auch im Hinblick auf die Tonalität einzelner Daten einer manuellen und damit, dem Verständnis der Befragten nach, einer ,qualitativen “ Untersuchung unterzogen werden. Laut den praktischen Anwendern beinhalten Social-MediaAnalysen quantitative und, qualitative' Aspekte. Frequenzanalysen in Bezug auf die Häufigkeit der Nennung eines Suchbegriffes werden als quantitative Tonalitätsanalysen, Sentiment-Analysen hingegen als ,qualitative“ Tonalitätsanalysen eigeordnet. Die Bezeichnung "qualitativ“ gründet in der manuellen Codierung, die die Befragten als besonderes Merkmal der Social-Media-Analysen mit Agentur $(\mathrm{X})$ betonen. Einige Praktiker sprechen in diesem Kontext fälschlicherweise von „Clusteranalysen“ und beschreiben dann Tonalitätsanalysen als ein manuelles Durchgehen der Daten und deren ,intelligente Bündelung“ zu Themenkomplexen. Sie setzen die manuelle Tätigkeit mit einem ,intelligenten“ Vorgehen gleich und nennen dies ,qualitativ“. Hier zeigt sich zum wiederholten Male ein eklatanter Mangel an der Kenntnis sozialwissenschaftlicher Forschungsmethoden, auf Seiten der Befragten sowie auf Seiten der Agentur, die ihre Dienstleistung offenbar entsprechend vermarktet. Wären fundierte Kenntnisse sozialwissenschaftlicher Forschungsmethoden bei den Befragten vorhanden, würde das Vorgehen von Agentur (X) entweder, sofern die Rede von „Frequenzanalyse“ ist, „quantitative Inhaltsanalyse" genannt werden oder bei einer Thematisierung von Tonalitätsanalysen als „qualitative Inhaltsanalyse“ bezeichnet werden; ferner wäre noch der Bericht von einer Kombination beider Verfahren denkbar. Bevor Agentur (X) mit einer Social-Media-Analyse beginne, führe sie einen Pre-Test durch, um zu prüfen, ob hinsichtlich der Fragestellung und der vereinbarten Parameter mit Ergebnissen zu rechnen ist. Die Ergebnisse des Pre-Tests würden von dem Dienstleister und dem Unternehmen besprochen und nur in dem Fall, dass er positiv ausgefallen sei, beginne die eigentliche Umsetzung.

Ergebnisse einer Social-Media-Analyse, so zeigen die Berichte der Praktiker, liefert die Agentur in Form eines Abschlussberichts beziehungsweise einer Ergebnispräsentation. Darin sei die der Untersuchung zugrunde liegende Fragestellung zusammengefasst und seien die den sozialen Medien entstammenden, bezüglich der Fragestellung relevanten einzelnen Kommentare in Gestalt aggregierter „Interpretationspanels" gebündelt. Ferner seien weiterführende Hypothesen enthalten. Agentur (X) würde die Ergebnisse nicht nur in Gestalt eines Abschlussdokuments liefern, sondern diese wie auch die Einzelbeiträge aus Social Media in einem 
Dashboard zusammenführen und dem Unternehmen einen Zugang zu diesem bereitstellen. Aus Basis dieser könnten die anwendenden Praktiker auf einzelne aus sozialen Medien erhobene Daten und damit Originalaussagen zugreifen und selbst weitere Auswertungen vornehmen. Inwiefern dieses Angebot genutzt wird, geht aus dem untersuchten Datenmaterial jedoch nicht hervor.

In den Berichten einiger Befragter bezüglich einer Zusammenarbeit mit Agentur (X) sticht hervor, dass es ihnen schwer fällt oder unmöglich erscheint, das methodische Vorgehen der Agentur bei der praktischen Durchführung von SocialMedia-Analysen zu erklären. Begründet wird dieses Unwissen einerseits damit, dass ein Erinnern an einzelne Untersuchungen unmöglich sei, da deren Umsetzung weit in der Vergangenheit stattgefunden habe, andererseits mit der Aussage, dass man an deren Umsetzung nicht teilgenommen habe.

Einige Befragte weisen in Bezug auf Agentur (X) außerdem darauf hin, dass es sich bei diesem Dienstleister um eine mittelständische Agentur handelt, deren Mitarbeiter sich durch Diversität bezüglich ihrer kulturellen Herkunft und damit auch Muttersprachen auszeichnen. So würden die Daten von Muttersprachlern mit Marktkenntnissen codiert und ausgewertet.

Anhand des Untersuchungsmaterials sind nicht nur Gemeinsamkeiten in der Umsetzung von Social-Media-Analysen durch Agentur (X) zu erkennen. Ebenso sind Unterschiede zwischen den einzelnen Unternehmenseinheiten, die Social-Media-Analysen bei dem Dienstleister abrufen, festzustellen. Die SocialMedia-Analysen werden mitunter nicht für Fragestellungen der eigenen Abteilung umgesetzt, sondern für den Bereich F\&E gebündelt. Es sei dann die Aufgabe einer Abteilung mit Schnittstellenfunktion andere Fachbereiche des Unternehmens im Sinne einer internen Dienstleistung in Bezug auf die Anwendung von Methoden zu beraten; gegebenenfalls werde eine Social-Media-Analyse abgerufen. Voraussetzung dafür sei zunächst, dass Fachbereiche, die an Social-Media-Analysen interessiert sind, an die Schnittstellenabteilung herantreten. Daraufhin würde die Beratung bezüglich der methodischen Passung einer Social-Media-Analyse für die jeweilige Fragestellung des Fachbereichs erfolgen. Ferner habe sich ein Fachkreis etabliert, in dem Anfragen für Social-Media-Analysen ebenfalls diskutiert werden. Wenn ein interessierter Fachbereich über das erforderliche Budget verfüge, werde gelegentlich eine Social-Media-Analyse durchgeführt. Meist wird gemäß den Befragten von der Durchführung abgeraten. Würde die Beauftragung von Agentur (X) befürwortet, wird eine Erstvermittlung zwischen dem Fachbereich und der Agentur geleistet. Gemeinsam würden dann die Details der Untersuchung besprochen und die Fragestellung ausgearbeitet. Die Ergebnisse werden im erwähnten Fachkreis präsentiert und von dem Fachbereich, der die Umsetzung 
initiiert hat, weiterverwendet. Es komme vor, dass sich Fachbereiche einer Empfehlung der Schnittstellenabteilung widersetzen und Social-Media-Analysen trotz ihres Abratens in Auftrag geben; eine Empfehlung ist unverbindlich. Wegen des damit einhergehenden Anstiegs der Umsetzung von Social-Media-Analysen und eines personellen Engpasses der Schnittstellenabteilung müssten die Fachbereiche die Administration selbst übernehmen, was wiederum den Anstieg der Freischaltungen von Fachbereichen für den bestehenden Rahmenvertrag zur Folge habe. Ferner würden Fachbereiche direkt mit Agentur (X) in Kontakt treten. Agentur (X) informiere dann die Abteilung mit Schnittstellenfunktion über die Anfragen und hole sich deren Einverständnis für die Umsetzung der Social-Media-Analysen ein.

Eine weitere Besonderheit der Umsetzung von Social-Media-Analysen mit Agentur (X) besteht darin, dass sich sechs für Qualitätsmanagement zuständige Fachbereiche des F\&E-Bereichs für einen eigenen Rahmenvertrag zusammengeschlossen haben. Für diesen Vertrag, der seit circa drei Jahren besteht, werde zu Jahresbeginn von jedem Fachbereich Budget eingeplant, so dass SocialMedia-Analysen im Jahresverlauf problemlos und kurzfristig bei der Agentur, die „Gewerkenehmer“ genannt wird, abgerufen werden können. Die Resultate dieser Social-Media-Analysen würden in einer für alle beteiligten Fachbereiche zugänglichen Datenbank gespeichert, so dass vor der Beauftragung einer Social-Media-Analyse überprüft werden kann, ob schon Ergebnisse zu der aktuellen Fragestellung oder auch verwandten Themen vorliegen. Alle an diesem Rahmenvertrag beteiligten Fachbereiche haben laut den Praktikern in Bezug auf Social-Media-Analysen bislang ausschließlich Erfahrungen mit Agentur (X) gesammelt. Deren Leistung habe überzeugt, so dass nicht mit anderen Dienstleistern experimentiert wurde. Social-Media-Analysen ordnen die Befragten im Kontext ihres Rahmenvertrags als ein „reaktives“ Instrument ein. Damit meinen sie, dass mit ihren Social-Media-Analysen Reaktionen, Meinungen und Rückmeldungen aus sozialen Medien zu den auf dem Markt angebotenen Produkten eingeholt werden. Da die Automobilindustrie durchweg schon an Folgegenerationen der aktuell am Markt erhältlichen oder aber an gänzlichen neuen Produkten arbeitet, würden mit Social-Media-Analysen entweder Reaktionen zu eigenen Vorgängergenerationen oder auch zu aktuellen Angeboten des Wettbewerbs eingeholt. Darauf könne der F\&E-Bereich des deutschen Automobilherstellers dann reagieren. Social-Media-Analysen würden Frequenzanalysen, Tonalitätsanalysen und Clusteranalysen umfassen. Methodisch würde zunächst ein ,grobes Herangehen" an das zu untersuchende Thema beziehungsweise die Fragestellung erfolgen; diese münde in ein ,iteratives Tieferlegen“. Mit Letzterem ist eine Auswertung der erhobenen Daten nach im Vorfeld benannten Themen gemeint. Weiterhin 
berichten die Befragten, dass stets ein Projektleiter des beauftragenden Fachbereichs die Social-Media-Analysen unternehmensintern begleitet, diese mit einem Workshop beginnen und enden. In einem „,initialisierenden Kick-Off-Workshop“ werde Agentur (X) ,inthronisiert“, in einem „Abschluss-Workshop“ präsentiere der Dienstleister seine Ergebnisse. Der Workshop am Ende einer Social-MediaAnalyse diene zum einen der Abnahme und Freizeichnung der Ergebnisse, zum anderen finde dieser statt, um offene Fragen durch Agentur $(\mathrm{X})$ beantworten zu lassen. In Bezug auf die „Inthronisierung“ wird darauf hingewiesen, dass es für den unternehmensinternen Umgang mit den Ergebnissen entscheidend ist, welche Person eines Fachbereichs die Social-Media-Analysen leite. Es sei wichtig, dass ein Projektleiter seinen Fachbereich im Vorfeld der Durchführung einer Social-Media-Analyse dafür sensibilisiert, dass die Methode kritische Rückmeldungen zu Produkten liefern kann und diese nicht übergangen werden dürfen. Für den Erfolg von Social-Media-Analysen sei es entscheidend, dass diese innerhalb des Unternehmens durch sozialkompetente Projektleiter begleitet werden. Diese müssten den Umgang mit kritikunfähigen Kollegen aus den Fachbereichen beherrschen. Neben einer persönlichen Kritikfähigkeit seien des Weiteren eine Affinität zu sozialen Medien oder zumindest Offenheit dafür und Reflexivität darauf unabdingbare Voraussetzungen für eine Leitung der Umsetzung von Social-Media-Analysen.

Das letzte Fallbeispiel der Umsetzung von Social-Media-Analysen mit Agentur (X), das sich durch seine Differenz zu anderen Berichten auszeichnet, beruht auf Erfahrungen, die Praktiker der Abteilung für Innovationsmanagement gemacht haben. Diese hat mit Agentur (X) bereits mehrere Social-Media-Analysen im Hinblick auf Fragestellungen zu Produkten durchgeführt. Die angewandte Praxis zeichnet sich insbesondere dadurch aus, dass die Daten aus marktspezifischen, inhaltlich relevanten Foren erhoben und nach bestimmten, vor der Untersuchung festgelegten Themenkomplexen codiert werden. Als Beispiele nennen die Befragten hier Maßnahmen zur Optimierung der Produktqualität, Erkenntnisse in Bezug auf technische Zusatzfunktionen sowie auch konkrete Fahrzeuge. Zum einen seien Themen im Kontext des Innovationsmanagements von Interesse; zum anderen werde nach Innovationen beziehungsweise Hinweisen auf solche gesucht.

Die Social-Media-Analysten betonen in Bezug auf Agentur (X) allem anderen voran, dass sich der Dienstleister durch sein manuelles, nicht vollautomatisiertes Vorgehen auszeichnet. Zwar ist dieses aufgrund der, qualitativen“ Inhaltsanalyse als Bestandteil der Methode wohl zu verzeichnen, doch gehen einige Praktiker fälschlicherweise davon aus, dass sämtliche, über den eigenen Crawler erhobenen Daten im Anschluss von Menschen codiert und einer ,qualitativen 
Inhaltsanalyse unterzogen werden. Vielmehr ist anzunehmen, dass eine Vorsortierung der aus sozialen Medien erhobenen Daten durch das Softwareprogramm der Agentur geleistet und mithilfe dessen letztlich auch eine Stichprobe der Gesamtdatenmenge für die ,qualitative' Inhaltsanalyse oder eben die in Anlehnung daran durchgeführte Codierung, die „menschliche“ Analysten manuell umsetzen, gezogen wird. Somit beinhalten die von Agentur (X) durchgeführten Social-Media-Analysen zwar durchaus manuelle oder, wie die Befragten sich ausdrücken, „qualitative“ Anteile, doch sobald ein Vergleich mit anderen Dienstleistern angestellt wird, sind diese nicht von nennenswerter Höhe. Das wird sich im Verlauf dieser Arbeit noch zeigen. Was Agentur (X) gemäß den Praktikern von alternativen Dienstleistern unterscheidet, ist das zur Verfügung gestellte Dashboard und die Zusammenführung von Ergebnissen einzelner Social-MediaAnalysen, die mehrere Abteilungen beauftragt haben können. Das Dashboard ermöglicht es den Befragten auf die aus sozialen Medien erhobenen Daten und damit auf Originalaussagen zuzugreifen und eigenständig weitere Auswertungen vorzunehmen. Jedoch weisen weder die praktischen Anwender fundierte Kenntnisse sozialwissenschaftlicher Forschungsmethoden auf, noch scheint Agentur (X) über solche zu verfügen. Letzteres ist aufgrund der methodisch falschen Bezeichnungen und Erläuterungen anzunehmen.

\subsection{Social-Media-Analysen mit Agentur $(\mathrm{Y})$}

Agentur (Y) ist ein weiterer Dienstleister, mit dem bereits einige Praktiker Erfahrungen in der Durchführung von Social-Media-Analysen gesammelt haben. Die Umsetzung von Social-Media-Analysen mit Agentur $(Y)$ weist zwar manche Ähnlichkeiten mit der zuvor thematisierten Durchführung durch Agentur (X) auf, aber auch etliche Unterschiede, auf denen das Hauptaugenmerk der folgenden Ausführungen liegt.

Die Zusammenarbeit mit Agentur (Y) ist aus eigenständig praktizierter Desk Research hervorgegangen. Zunächst sei über eine manuelle Recherche ermittelt worden, was in sozialen Medien geschrieben wird, und dann wegen relevanter Erkenntnisse ein tieferer Einstieg in das Thema mit dem Dienstleister erfolgt. Dessen Vorgehen bestehe im „Anzapfen“ von Foren sowie Text Mining und habe sich mit der Zeit professionalisiert. Im Vergleich mit den zuvor thematisierten Social-Media-Analysen in Kooperation mit Agentur (X) machen die Befragten, die Erfahrungen mit Agentur (Y) schildern, noch unspezifischere Angaben zur methodischen Umsetzung von Social-Media-Analysen. So entsteht der Eindruck, dass der Agentur ein fast ,blindes Vertrauen " entgegengebracht wird; weder liegen Kenntnisse über die hinter deren Untersuchungen stehende Methodik vor, noch wird ihr Vorgehen hinterfragt. Mit dieser Vermutung verbunden ist eine 
weitere Annahme: Dass nämlich die Praktiker, die von Erfahrungen mit Agentur (Y) berichten, keine fundierten Kenntnisse sozialwissenschaftlicher Forschungsmethoden besitzen. Wären solche vorhanden, wäre wohl eine Auseinandersetzung mit der hinter den Social-Media-Analysen stehenden Methodik längst erfolgt und von dieser berichtet worden. Mit dem Dienstleister würden seit Beginn der Zusammenarbeit Social-Media-Analysen zu zwei Zwecken praktiziert; einerseits begleitend zur Markteinführung neuer Produkte, andererseits zum Aufdecken von Produktfehlern. Das Unternehmen erstelle vor jeder Social-Media-Analyse ein Briefing, in dem es die Erwartungshaltung an die Agentur kommuniziert. Agentur $(\mathrm{Y})$ würde basierend auf diesem mit der Umsetzung beginnen, nach circa drei Wochen eine Ergebnispräsentation liefern, diese bei Bedarf überarbeiten und zum Abschluss dem Unternehmen zusenden; die Präsentation sei professionell ausgearbeitet und enthalte Screenshots der sozialen Medien.

Befragte aus der CRM-Abteilung schildern ihre Erfahrungen mit Agentur (Y) in Bezug auf die, von ihnen „Performance Tracking“ genannte Variante. In deren Rahmen werde beispielsweise analysiert, wie viele Nutzer von Social Media auf eine Werbeanzeige des Unternehmens bei Facebook geklickt haben, welche Anzahl dieser sich danach auf der Webseite des Unternehmens registriert hat, wie viele dieser registrierten Nutzer Influencer oder „Leads“ wurden, die an den Handel weitergeleitet wurden, und wie viele der weitergeleiteten Influencer letztlich ein Produkt des Automobilherstellers gekauft haben. Aus dem Untersuchungsmaterial geht hervor, dass in der Abteilung für CRM neben „Performance Tracking“ auch Social Media Listening praktiziert wird. Laut der Befragten geht es beim Listening darum, welche Inhalte in sozialen Medien besprochen werden und in welcher Tonalität dies geschieht:

Befragter: [. . . ] Das andere ist das Thema, was wird denn so gesprochen? Ähm, und da wir die Dialog-Komponente [. . . ] sind, zum Beispiel Abgas-Skanda/, ähm, AbgasSkandal als Beispiel, müssen wir auch wissen, was wird denn im CIC, also im Customer Interaction Center, was kommt da an Traffic rein? Worüber wird da gesprochen? Und auch im Social Media. Was sprechen die denn auf Motortalk, Facebook, Going Electric [... ]? Was weiß ich. Was wird denn da gesprochen? Und in welcher Tonalität wird es gesprochen? Also, das eine ist sozusagen die, ähm, Performance-Analyse, das andere ist das Thema, ähm, Social Media Listening. Was wird gesprochen und in welcher Tonalität wird es gesprochen (I12/ S2/ Z10-17)?

Mit „Performance Tracking“ oder auch „Performance-Analyse“ sowie Social Media Listening spannen die Praktiker ein eigenes Kontinuum an Social-MediaAnalysen auf. Ein Ende dieses Kontinuums erinnert mit „Performance Tracking“ an eine quantitative Inhaltsanalyse, da es hier in erster Linie um die Ermittlung 
von Frequenzen geht; am anderen Ende des Kontinuums erfolgt mit Social Media Listening durch die Ermittlung der eigentlichen Inhalte, auch der nicht unmittelbar artikulierten ,zwischen den Zeilen', eine Assoziation mit qualitativen Inhaltsanalysen. Das Ziel besteht laut den Befragten darin, Verständnis zu erzeugen. Zwar zieht kein Praktiker derartige Vergleiche mit den Methoden der Sozialforschung oder stellt Verbindungen zu diesen her, doch im Rahmen dieser Arbeit muss angemerkt werden, dass dies möglich oder vielmehr naheliegend gewesen wäre. Dafür sind allerdings entsprechende Kenntnisse vonnöten. Den Prozess der Umsetzung von Social-Media-Analysen stellen die Befragten auch wieder nur sehr oberflächlich dar. Im Vorfeld von Social-Media-Analysen seien gemeinsam mit der Agentur verschiedene Kategorien für die Sortierung der erhobenen Daten festgelegt worden. Exemplarisch werden als Kategorien Modellbezeichnungen, Anfragen an den Service, Aussagen zu dem deutschen Automobilhersteller und dessen Hauptmarke sowie Alarmsignale allgemein genannt. Aktuellen Situationen oder Fragestellungen entsprechend würden ad hoc weitere Kategorien, „Verschlagwortungen“ genannt, hinzugenommen, etwa bei Rückrufaktionen. Agentur (Y) setze für die Datenerhebung einen Algorithmus beziehungsweise ein eigenes Tool ein. Dieses sei sowohl mit den allgemeinen als auch bei einer Ad-hoc-Fragestellung mit zusätzlichen Kategorien angereichert und würde Social Media durchforsten. Anders als bei Agentur (X) besteht bei Agentur (Y) laut den Befragten die Möglichkeit einen eigenen Zugriff zu dem Tool zu bekommen, so dass darüber Daten zwar aus sozialen Medien erhoben, jedoch nicht von der Agentur ausgewertet werden. Das Tool ermittle nur die Daten und biete einen Zugriff auf diese, die Auswertung der Daten würden die praktischen Anwender des Unternehmens selbst vornehmen. Hierbei handelt es sich um eine Kombination der Umsetzung von Social-Media-Analysen mit einer Agentur über die Nutzung deren Tools und eine eigenständige Auswertung, die mit der Umsetzung über Desk Research zu vergleichen ist. Alternativ könne neben der Datenerhebung auch deren Auswertung bei dem Dienstleister beauftragt werden. Diese sei insbesondere in Bezug auf ,qualitative“ Analysen, die erhöhte Kosten verursachen und ein „Reingehen“ in einzelne Beiträge sozialer Medien erfordern, interessant. Auf diesem Weg würden zum Beispiel in Hinblick auf bestimmte Produkte ,qualitative “ Tonalitätsanalysen entweder von der Agentur durchgeführt oder unter Einsatz des eigenständig zu bedienenden Tools von den befragten Praktikern. Letzteres vor allem mit quantitativem Fokus, wenn die Entwicklung von Themen mehr interessiert als deren inhaltliche Details. Ergebnisse der von Agentur (Y) durchgeführten oder mithilfe von deren Tools umgesetzten Social-Media-Analysen werden dem 
jeweiligen Führungskreis des Unternehmens mitgeteilt. Vor allem bei Ad-hocFragestellungen würde dieser die aktuellen Entwicklungen im Hinblick darauf verfolgen, ob akuter Handlungsbedarf besteht.

Die Berichte der Befragten über die Zusammenarbeit mit Agentur (Y) sind im Vergleich mit der zuvor behandelten Kooperation mit Agentur (X) von oberflächlicher Natur. Dies mag auch darin begründet sein, dass mehr SocialMedia-Analysten über Erfahrungen mit Agentur $(X)$ verfügen. Dennoch können den Schilderungen der Befragten einige Aspekte entnommen werden, die die von Agentur (Y) durchgeführten Social-Media-Analysen auszeichnen. Dabei handelt es sich vorranging um deren Tool, welches nicht nur in einem Dashboard besteht und erhobene und von der Agentur ausgewertete Social-Media-Daten beinhaltet, sondern um eines, mit dem von den Praktikern selbst Daten erhoben werden können. Social-Media-Analysen können von der Datenerhebung bis zur Ergebnispräsentation zwar komplett bei Agentur (Y) beauftragt werden, dennoch stellt die Möglichkeit der selbstständigen Durchführung dieser über das Tool eine Alternative dar, die vor allem bei begrenztem Budget interessant sein kann. Im Zusammenhang der Berichte der praktischen Anwender zur Durchführung von Social-Media-Analysen mit Agentur (Y) scheint zudem vor allem das von ihnen aufgespannte Kontinuum zwischen einer eher quantitativen Variante von Social-Media-Analysen als „Performance Tracking“ und der ,qualitativeren “ Variante des Social Media Listenings interessant. Beide Ansätze erinnern an die der Sozialforschung entstammende Methode der Inhaltsanalyse, einerseits an deren quantitative, andererseits an deren qualitative Ausprägung. Allerdings haben die Social-Media-Analysten dies so nicht ausgeführt und damit vermutlich auch nicht erkannt. Erst recht haben sie die hinter den Social-Media-Analysen stehende Methodik nicht hinterfragt, was erneut den Mangel an Kenntnissen sozialwissenschaftlicher Forschungsmethoden deutlich macht. Ferner ist zu vermuten, dass die praktischen Anwender des Automobilherstellers die Social-Media-Analysen nicht reflektiert, sondern unhinterfragt mit Agentur (Y) umsetzen.

\subsection{Social-Media-Analysen mit Agentur (Z)}

Mit Agentur (Z) hat sich im Untersuchungsmaterial neben den bereits thematisierten ein dritter Dienstleister herauskristallisiert, mit dem die praktischen Anwender von Social-Media-Analysen ihre Erfahrungen gesammelt haben. In den Berichten der Befragten sind in Bezug auf die Umsetzung von Social-Media-Analysen mit Agentur (Z) ebenfalls Gemeinsamkeiten wie Unterschiede zu verzeichnen. Erneut wird in einem ersten Schritt die Schnittmenge vorgestellt, welche sich aus den Ausführungen im Hinblick auf die Zusammenarbeit mit Agentur (Z) beziehungsweise die Umsetzung von Social-Media-Analysen durch diesen Dienstleister 
ergeben hat. Anschließend werden Erfahrungen der Social-Media-Analysten des deutschen Automobilherstellers bezüglich der Umsetzung durch diese Agentur, die sich deutlich voneinander unterscheiden, behandelt.

Eine Gemeinsamkeit der Befragten, die von der Kooperation mit Agentur (Z) berichten, besteht darin, dass sie dem Unternehmensbereich für Qualität angehören und Social-Media-Analysen ausschließlich von Dienstleistern umsetzen lassen. Dies bedeutet, dass die Praktiker Social-Media-Analysen niemals eigenständig praktizieren, etwa über Desk Research oder mit Tools, wie dies manche Befragte tun, die von der Zusammenarbeit mit anderen Agenturen berichten. Mit dieser Tatsache einher geht die uneingeschränkte Anerkennung von Fachwissen in Bezug auf Social-Media-Analysen mit Agentur $(Z)$ und die Bewertung ihres Vorgehens als professionell. Zum einen attestieren Befragte Agentur (Z) zwar Fachwissen in Bezug auf Social-Media-Analysen und Professionalität bei deren Umsetzung, weisen aber auch darauf hin, dass sie diese Faktoren selbst nicht besitzen oder diese bei ihnen nicht ausgeprägt sind. Letzteres wird deutlich, da man den methodischen Vorzügen von Social-Media-Analysen, die auch in deren explorativem und offenem Charakter bestehen, von Beginn an mit festgeschriebenen Quantitäten im Hinblick auf erst noch zu ermittelnde Ergebnisse begegnet.

Die Beauftragung von Agentur $(\mathrm{Z})$ erfolgt, anders als bei Agentur $(\mathrm{X})$, nicht über einen Rahmenvertrag, der das Abrufen einer Social-Media-Analyse im Bedarfsfall ermöglicht, sondern durch einen Vertrag über drei Jahre hinweg. Ihren Fokus haben die Befragten, so berichten sie, bei der Auswahl des Dienstleisters für diesen Vertrag vor allem auf den Einbezug „menschlicher“ Expertise in die Datenauswertung gerichtet. Agentur $(Z)$ verfüge zwar über ein eigenes Tool für die Umsetzung von Social-Media-Analysen, die Auswahl der Agentur sei jedoch nicht in der Qualität ihrer Software begründet. Es sei vielmehr ausschlaggebend gewesen, dass der Dienstleister die Daten manuell auswertet, also ein „menschlicher“ Analyst diese liest, strukturiert und nach der Untersuchung fertige Ergebnisse an den Automobilhersteller übergibt. Die Befragten schreiben der Erfahrung in manueller Codierung im Zuge der Datenauswertung große Bedeutung zu. Neben den Erwartungen, die an die Erfahrungen beziehungsweise das darauf beruhende Wissen der Agentur bezüglich SocialMedia-Analysen herangetragen werden, besteht zugleich auch Unwissen der Praktiker des Automobilherstellers im Hinblick auf die Methode. Erneut greift der Code „Agenturvertrauen“. Ein weiterer, bedeutsamer Aspekt bei der Auswahl der Agentur sei es gewesen, wie die Daten ausgewertet werden, die auf Social-Media-Plattformen in anderen Sprachen als Deutsch vorliegen. 
Die von der Agentur durchgeführten Social-Media-Analysen setzen sich, so führen die befragten Social-Media-Analysten aus, aus quantitativen und ,qualitativen' Aspekten zusammen. Deren Umsetzung durch Agentur $(Z)$ gehe ein „detailliertes“ Briefing durch den Projektleiter des Unternehmens voraus. In einem solchen werde die Fragestellung ebenso wie der Untersuchungszeitraum und die einzubeziehenden Märkte oder vielmehr Sprachräume festgesetzt. Die Aufgabe des unternehmensinternen Projektleiters bestehe in der Steuerung des Dienstleisters. Diesem müsse vermittelt werden, worauf bei Social-Media-Analysen zu achten ist; beispielsweise benötige er Informationen zu Produkten, deren Wahrnehmung untersucht werden soll. Zudem würden im Vorfeld einer Social-MediaAnalyse gegenüber Agentur $(\mathrm{Z})$ die Erwartungen an die Ergebnispräsentation kommuniziert. Nach inzwischen einigen mit dem Dienstleister durchgeführten Social-Media-Analysen verfüge dieser heute neben ihrem Methodenwissen ebenso über ein spezifisches Fachwissen und Produktwissen der Automobilindustrie; dies begünstige künftige Untersuchungen. Ferner hätte das Unternehmen gemeinsam mit Agentur (Z) zu Beginn der Zusammenarbeit ein Kategorienschema entwickelt, anhand dessen die Daten ausgewertet werden. Für die Datenerhebung, die auch hier „Crawling“ oder „Crawlen“ genannt wird und über ein von Agentur (Z) selbst entwickeltes Tool erfolgt, würden die Computerlinguisten der Agentur Suchanfragen formulieren. Letztere bezeichnen die Praktiker auch als „Vercodungen“ oder aber "Such-Terms“ und erklären, dass sie aus Textbausteinen bestehen und sich über mehrere Seiten erstrecken. Mit dem Tool würden die sozialen Medien durchsucht und ein zu untersuchender „Kommunikationsstrang" erhoben, der auch weitere Themen und Aspekte enthalten kann, die für Social-Media-Analyse ursprünglich zwar nicht angedacht waren, in der Datenauswertung jedoch ebenso beachtet werden müssen. Die Praktiker berichten weiterhin, dass nicht alle derart erhobenen Daten ausgewertet werden, sondern Agentur ( $Z$ ) daraus eine Stichprobe für die manuelle Codierung anhand des vorab feststehenden Kategorienschemas zieht. Das Kategorienschema setze sich aus mehreren „Code-Levels“ oder "Codeebenen“ je nach Thema und genauen Inhalten zusammen; Kategorien sind gemeint. Damit erinnern auch die SocialMedia-Analysen von Agentur $(Z)$ an quantitative Inhaltsanalysen, die sich von den vorher beschriebenen vor allem durch die manuelle Codierung der erhobenen Daten anhand der Kategorien unterscheiden. Trotzdem wird auch von den mit Agentur (Z) zusammenarbeitenden Praktikern, die das methodische Vorgehen und auch die praktische Durchführung des Dienstleisters mitunter wesentlich genauer zu berichten wissen als Befragte, die über Kooperationen mit anderen Agenturen sprechen, die Methode nicht entsprechend benannt. Dies zeigt, dass zwar eine Auseinandersetzung mit der Methode stattgefunden hat, jedoch auch 
hier davon auszugehen ist, dass dieser keine fundierten Kenntnisse des sozialwissenschaftlichen Methodenkanons zugrunde lagen, zumindest nicht auf Seite der befragten Social-Media-Analysten. Außerdem ist im Kontext quantitativer Verfahren abermals die Rede von „Frequenzanalysen“. Im Kontrast dazu werden auch in Bezug auf Agentur (Z) Tonalitätsanalysen wieder als ,qualitativer Bestandteil von Social-Media-Analysen charakterisiert. Diese würden genutzt, um Auffälligkeiten in sozialen Medien zu ermitteln. Ein weiteres Merkmal der von Agentur (Z) durchgeführten Social-Media-Analysen besteht nach Ansicht der Social-Media-Analysten darin, dass der Dienstleister die Ergebnisse mit Textbeispielen, sogenannten „Codings“, verdeutlicht. Die Ergebnissen würden um originale, sozialen Medien entnommene Inhalte in Textform ergänzt.

Neben den thematisierten Gemeinsamkeiten, die sämtliche Berichte der Befragten über die Kooperation mit Agentur (Z) in Bezug auf die Durchführung von Social-Media-Analysen eint, finden sich im Datenmaterial ebenso Unterschiede. Befragte erzählen, dass sie in der Vergangenheit bereits mit unterschiedlichen Anbietern von Social-Media-Analysen zusammengearbeitet haben, um von all diesen etwas über die Methode zu lernen. Dienstleister würden sich im Hinblick auf ihre Stärken erheblich voneinander unterscheiden. Nach dem Sammeln von Erfahrungen mit zwei Agenturen, (X) und (Y), kooperieren die Praktiker nun mit Agentur (Z). Der Dienstleister aggregiere „Konzept-Feedback“, also Rückmeldungen zu Produktkonzepten auf Social-Media-Kanälen, und würde circa vier Monate nach Markteinführung ein konsolidiertes Bild davon an den Automobilhersteller übergeben. Der Grund für die Umsetzung dieser Art von Social-Media-Analysen sei die Früherkennung von Fehlern bei Produkten und Problemen mit diesen. Diese für weitere drei Jahre bei Agentur ( $Z$ ) in Aufrag gegebene Social-Media-Analyse nennen die Befragten „Launch Monitoring“:

Befragter: Mhm (überlegend). Vielleicht mal was diese/, was dieses Launch Monitoring betrifft, äh, da gehen wir so vor/, also, wir haben ja grundsätzlich immer eine Agentur beschäftigt, ähm, (...) die diese Analysen durchführt, aber vielleicht muss man es unterteilen in diesen, äh, Launch Monitoring (...). Da funktioniert es ja so, dass die quasi auch ein Tool haben, das, äh, mehr oder weniger gut ist. Wir lesen auch nie das gesamte Internet, sondern tatsächlich auch, ähm, konzentrieren wir uns auf im Wesentlichen auf Foren und Blogs und, ähm, unser Fokus liegt schon (...) auf dem Kunden-Feedback, also auf (stockt), äh, auf Posts, die, äh, aufgrund des Kontextes darauf schließen lassen, dass jemand halt das Fahrzeug fährt oder Probe gefahren ist oder wie auch immer. Um dieses Feedback dann abzugreifen, dann, äh, (...), dampft die Agentur, wenn man so will, erstmal diese Unmengen an Daten ein und dann sitzt einfach einer und liest und strukturiert dann nach unserer Vorgabe die Daten und bringt die in so eine [Logik der Marke des Unternehmens des Befragten] [. . . ] (I5/ S9/ Z33-44). 
Die im Interviewausschnitt beschriebene Vorgehensweise entspricht weitestgehend der vorab dargestellten Kooperation mit Agentur (Z). Im Fokus des „Launch Monitorings" stehen Foren des englischen, deutschen, chinesischen, japanischen, koreanischen und - für ein ganz spezielles Produkt - auch des norwegischen Sprachraums, die mitunter Tonalitätsanalysen unterzogen werden.

Neben dem „Launch Monitoring“, so berichten die Praktiker weiter, werden zusätzlich als solche bezeichnete „Tiefenanalysen“, die sich durch ihre Retrospektive auszeichnen, umgesetzt. Zum Einsatz kämen diese für Ad-hocFragestellungen, welche im Rahmen des regulär stattfindenden „Launch Monitoring" nicht beantwortet werden können. Die in Bezug auf das bestehende Kategorienschema codierten Daten würden auf spezifische Fragestellungen hin gesondert ausgewertet:

Befragter: Und, ähm, (...). Genau. Bei den Tiefenanalysen da geht es jetzt ja weniger darum, dass wir quasi, ähm, fortlaufend mit, ähm, (stockt) schauen, wie so einzelne, äh, wie so ein Fahrzeug beurteilt wird. Da guckt man rückblickend ins Netz, was es zum Beispiel zum Head Up Display, ähm, über verschiedene Fahrzeuge hinweg, ja, was gibt es da für Feedback? (I5/ S11/ Z35-38).

„Tiefenanalysen“ seien demnach durch eine konkrete Fragestellung auf „Komponenten-Ebene“ oder „Funktions-Ebene“ einzelner Produkte gekennzeichnet, die betreffend die in sozialen Medien vorhandene Wahrnehmung untersucht wird. Die Praktiker fassen den Unterschied zwischen dem mit Agentur (Z) umgesetzten „Launch Monitoring“ und den ebenfalls von diesem Dienstleister durchgeführten „Tiefenanalysen“ so zusammen, dass das Vorgehen bei ersterem „offener“ sei. Beim „Launch Monitoring“ werde erst „offen“ ermittelt, welche Inhalte zu Produkten oder auch zu speziellen Fahrzeugen des Automobilherstellers in sozialen Medien zu finden sind, um Themenschwerpunkte zu erkennen und in von Beginn an feststehende Kategorien zu untergliedern. Ob es sich dabei tatsächlich um eine offene Herangehensweise an einen Untersuchungsgegenstand oder gar um eine explorative Vorgehensweise bei der Umsetzung der Untersuchung handelt, muss hier in Frage gestellt werden. Sozialwissenschaftliche Methodenliteratur beschreibt quantitative Inhaltsanalysen, mit denen die dargelegte Umsetzung von Social-Media-Analysen eine nicht abzustreitende Ähnlichkeit aufweist, aufgrund eines im Vorfeld erarbeiteten Codebuchs, das einem Kategorienschema entspricht, eher als geschlossene Methode; vor allem im Vergleich mit qualitativen Inhaltsanalysen. Ohne Zweifel gibt es zwar noch geschlossenere Verfahren als quantitative Inhaltsanalysen, doch es scheint im vorliegenden Kontext falsch, „Launch Monitoring“ als offene Art von Social-Media-Analysen 
einzustufen, vor allem nicht im Vergleich mit den ebenso beschriebenen „Tiefenanalysen“. Die Ergebnisse der beiden Arten von Social-Media-Analysen werden regelmäßig als erweiterter "Sensor“ an den Unternehmensbereich für Produktmanagement oder, sofern es sich um reines „Konzept-Feedback“ handelt, an die Vertreter einzelner Produktlinien des Automobilherstellers berichtet. Ferner würden die Ergebnisse in einen umfassenden „Launch Report“ integriert. Agentur (Z) liefere die Ergebnisse in einer gemeinsam erstellten Excel-Liste, die nach den schon angeführten Kategorien aufgebaut ist; die Ergebnisse der „Tiefenanalysen“ hingegen hätten die Gestalt eines Abschlussberichts und würden in einem Präsentationsformat bereitgestellt. Zudem schildern die Social-Media-Analysten, dass gemeinsam mit einem weiteren Anbieter an der Entwicklung eines eigenen Dashboards gearbeitet wird. Ein solches solle unabhängig entwickelt werden, um die Untersuchungsergebnisse auch nach dem Wechsel des Dienstleisters zusammenzufassend zu speichern und sich als Unternehmen nicht in eine Abhängigkeit zu begeben. Ferner würden die Ergebnisse regelmäßig mit den Early Buyers Studies der unternehmensinternen Marktforschung abgeglichen.

Andere Befragte berichten von mehreren über zwei Jahre hinweg umgesetzten Social-Media-Analysen mit Agentur (Z), in denen die in sozialen Medien vorherrschende Wahrnehmung bezüglich zwei spezifischer Fahrzeuge des Automobilherstellers erhoben wurden. Über Social-Media-Analysen sei der europäische Markt und der englische Sprachraum untersucht worden; auch der japanische Markt sei zu Beginn betrachtet, dann aufgrund von Übersetzungsschwierigkeiten und der Erkenntnis dessen Nicht-Dominanz jedoch wieder verworfen worden. Für die Datenerhebung wurden fachlich relevante Blogs forciert. Das Hauptinteresse der SocialMedia-Analysen habe darin bestanden, zu ermitteln, was in sozialen Medien zu einem frühen Zeitpunkt, zu dem noch keine kommunikative Beeinflussung stattgefunden hat, über die beiden neuen Produkte diskutiert wurde. Die Zusammenarbeit mit Agentur (Z) sei nach dem Prinzip „Learning by Doing“ erfolgt, da das Projekt sowohl für die Agentur als auch für das Unternehmen die erste gemeinsame Social-Media-Analyse dargestellt hat. Im Vorfeld der Untersuchung wurden Details, die mit der Social-Media-Analyse betrachtet werden sollen, gegenüber der Agentur kommuniziert. Die Agentur liefert die Ergebnisse der Social-Media-Analysen in Gestalt monatlicher Berichte, wobei die erste Ausgabe überarbeitet und den Erwartungen des Unternehmens angepasst werden musste. Die Praktiker bezeichnen die Ergebnisse als „Cluster“ und führen beispielhaft Märkte im Sinne von Verkaufsregionen an, die vorab als zu untersuchend festgesetzt wurden. Auf Nachfrage hin habe Agentur ( $\mathrm{Z}$ ) bezüglich bestimmter Themen, die sich in einer Social-MediaAnalyse als relevant herausgestellt haben, erneut analysiert und abschließend die Ergebnisse in einer Präsentation vorgestellt. Die Praktiker haben, so beschließen sie 
ihre Ausführungen, die Ergebnisse in entsprechende Fahrzeugprojekte ,hineingetragen" und dort ebenso wie in Managementkreisen vorgestellt; Letztere hätten einzelne Aspekte gezielt weiterverfolgt. Von Unternehmensseite hätten in erster Linie Praktikanten die Social-Media-Analysen betreut und deren Ergebnisse in Excel-Listen verwaltet. Anhand dieser sei ein eigenständiges und manuelles Durchsuchen ohne erneute Unterstützung durch Agentur (Z) bei Bedarf möglich gewesen. Dieses Vorgehen ist nicht mit einer im Nachhinein selbst durchgeführten Social-Media-Analyse gleichzusetzen.

Es ist festzuhalten, dass die Ausführungen der Befragten zu Social-MediaAnalysen in Kooperation mit Agentur (Z) vor allem bezüglich ihrer praktischen Durchführung und methodischen Umsetzung wesentlich detaillierter ausfallen als die Berichte, die sich im untersuchten Datenmaterial zu anderen Agentur, wie (X) und (Y), finden. Die Befragten streichen mehrere Aspekte als besondere Kennzeichen der von Agentur (Z) umgesetzten Social-Media-Analysen heraus. Es handelt sich zunächst um die mit dem Dienstleister realisierten Social-Media-Analysen als „Launch Monitoring“ und „Tiefenanalysen“. Außerdem betonen sie, dass SocialMedia-Analysen von Agentur (Z) unter Einsatz ihres eigenen Tools durchgeführt und die in das Tool eingegebene Suchanfragen von Computerlinguisten formuliert werden. Hauptmerkmal, darin sind sich die Praktiker einig, ist jedoch die von Agentur (Z) umgesetzte manuelle Codierung der aus sozialen Medien erhobenen Daten durch ,menschliche“ Analysten.

\subsection{Social-Media-Analysen mit Social-Media-Agenturen}

Für Social-Media-Analysen, die mit Dienstleistern durchgeführt werden, beauftragt der betrachtete Automobilhersteller allerdings nicht ausschließlich die drei vorgestellten, sondern kooperiert ferner mit zwei, von den Praktikern als solche bezeichneten ,Social-Media-Agenturen“ oder „Customer Service Agenturen“. Ein wesentlicher Unterschied zwischen Social-Media-Agenturen, (V) und (W), und Agenturen wie (X), (Y) und (Z) besteht darin, dass erstere nicht nur Social-Media-Analysen durchführen, sondern für eine umfassende Betreuung der Social-Media-Kanäle des Unternehmens beauftragt werden und diese um SocialMedia-Analysen ergänzen. Die praktischen Anwender, die von Social-MediaAgenturen berichten, stammen alle aus verschiedenen Marketing-Abteilungen des Unternehmens und haben doch nur mit zwei Social-Media-Agenturen ihre Erfahrungen gesammelt. Ihre Berichte über die Zusammenarbeit mit diesen beiden Dienstleistern ähneln sich sowohl in Bezug auf die inhaltliche als auch die methodische Komponente der Social-Media-Analysen und werden daher gemeinsam als Social-Media-Analysen mit Social-Media-Agenturen vorgestellt. 
Social-Media-Agenturen, die für den Automobilhersteller Untersuchungen durchführen, integrieren die Social-Media-Analysen in ihre Tätigkeit des Social Media Community Management. Das bedeutet, dass die Agenturen im Auftrag des Unternehmens dessen eigene nationale wie internationale Social-MediaPlattformen ,bespielen“ und diese außerdem, auch durch Nutzung ihrer integrierten Analysefunktionen, wie Facebook Insights, untersuchen. Die befragten Praktiker bewerten ihre Social-Media-Agenturen als für kleine Social-MediaAnalysen, die vor allem die eigenen Social-Media-Kanäle betreffen, geeignet. Zwar würden die Dienstleister vor allem die unternehmenseigenen Social-MediaPlattformen analysieren, in ihre Untersuchungen bei Bedarf jedoch auch weitere aufnehmen. Alternativ beauftragen die für Marketing zuständigen Abteilungen des Unternehmens laut den Befragten zusätzlich weitere Agenturen, (X), (Y) oder (Z). Diese untersuchen dann die Daten der eigenen und insbesondere auch der fremden Social-Media-Kanäle. Neben der gleichzeitigen Umsetzung von SocialMedia-Analysen durch verschiedene Dienstleister führen die Praktiker zudem noch eigene Social-Media-Analysen durch. Dies geschieht dann über den Einsatz von Tools oder durch Nutzung der in die eigenen Social-Media-Kanäle integrierten Analysefunktionen.

Social-Media-Analysen selbst werden im Rahmen der Durchführung mit Social-Media-Agenturen von den Befragten als zielgerichtete Abfragen in sozialen Medien anhand bestimmter Fragen definiert. Das Untersuchungsmaterial zeigt, dass die Social-Media-Agenturen ihre Untersuchungen in der Regel kontinuierlich und automatisiert umsetzen, also in festgesetzten Rhythmen, wiederholt zu gleichen Zeitpunkten und meist über den Einsatz von Softwares oder Tools. Beispielhaft nennen die Befragten mehrere Tools, wie (1), (2), (3) und (4). Hinzu kommen gelegentliche Sonderanalysen, die manuell umgesetzt werden. Begründet wird die Beauftragung von Social-Media-Agenturen für Social-MediaAnalysen vor allem mit der Notwendigkeit der Professionalisierung des Themas und damit, dass Social-Media-Analysen aggregierte Informationen, welche die Praktiker „Cluster“ nennen, liefern. Ein weiterer Beweggrund, der im Zusammenhang mit Marketing nahezu klassisch anmutet, ist es, Kampagnenerfolge messbar zu machen. Einige Befragte unterscheiden in ihrer Darstellung der Durchführung von Social-Media-Analysen mit Social-Media-Agenturen explizit zwischen zwei verschiedenen Ansätzen und zwar insofern, dass sie Listening und Monitoring als zwei Varianten anführen und Listening als kurzfristig beauftragte Sonderanalysen zu bestimmten Fragestellungen, Monitoring hingegen als wiederholendes Reporting beschreiben. Das Marketing des Unternehmens praktiziert Social-Media-Analysen vorrangig als Monitoring. Meist wird es automatisiert 
über Tools mit dem Fokus auf quantitative Ergebnisse durchgeführt. Demgegenüber wird Listening entweder über Software oder und dies häufiger manuell umgesetzt; die letztere Variante wird auch „intellektuelle“ Recherche genannt und als ,qualitative' Untersuchung eingestuft.

Den Prozess der Durchführung von Social-Media-Analysen in Kooperation mit Social-Media-Agenturen beschreiben die Social-Media-Analysten zum einen ziemlich ähnlich und zum anderen, in Bezug auf das gesamte dieser Forschungsarbeit zugrunde liegende Datenmaterial, überraschend detailliert. Allerdings gehen auch hier die Befragten nicht auf die hinter Monitoring oder Listening stehende Methodik ein, sondern konzentrieren sich auf die Beschreibung des Prozesses der Zusammenarbeit mit dem Dienstleister. Obwohl die Praktiker diesen ziemlich genau darstellen, ist nicht davon auszugehen, dass sie sich reflektiert mit methodischen Aspekten der ihrerseits beauftragten Social-Media-Analysen auseinandergesetzt haben. Die Berichte der Befragten über die Durchführung von Untersuchungen mit Social-Media-Agenturen lassen nicht auf fundierte Kenntnisse sozialwissenschaftlicher Forschungsmethoden schließen.

Social-Media-Analysen würden einerseits und vorrangig als quantitative Untersuchung über Tools beziehungsweise Software, andererseits auch als ,qualitative* in Form einer ,intellektuellen“ oder „redaktionellen“ Recherche durchgeführt. Allerdings machen die Praktiker in ihren Darstellungen des Prozesses von SocialMedia-Analysen kaum einen unmittelbaren Unterschied zwischen Monitoring und Listening aus. So ist anzunehmen, dass sich der beschriebene, hier nun dargestellte Umsetzungsprozess vor allem auf Monitoring bezieht, da dies verstärkt praktiziert wird. Gemäß der praktischen Anwender erfolgen im Vorfeld der Social-Media-Analysen, die die Marketing-Abteilungen zum Messen von Kampagnen und die „Beobachtung“ ihrer eigenen Social-Media-Kanäle einsetzen, Abstimmungen mit den Agenturen; im Fall von kontinuierlichem Monitoring in regelmäßigen Abständen. In diesen kommuniziert der Automobilhersteller seine Erwartungen an eine Untersuchung sowie deren Ergebnisse und definiert ferner gemeinsam mit der Social-Media-Agentur die zu untersuchenden Ziele einzelner Kampagnen, wie die Steigerung des Beitragsaufkommens in bestimmten sozialen Medien oder die Erhöhung der Reichweite einzelner Beiträge. Bei einer Social-Media-Analyse zur Messung von Kampagnen handle es sich um eine Untersuchung von begrenztem Umfang, die innerhalb eines bestimmten Zeitraums, zumeist ab Start einer Kampagne bis zu deren Abschalten, abgeschlossen werde. In einem nächsten Schritt nehme der Dienstleister eine „Nullmessung“ vor, in der das gegebenenfalls vorhandene „Grundrauschen“ in sozialen Medien, wie die Anzahl der Nennung bestimmter Begriffe oder Produkte, ermittelt wird. Diese „Nullmessung“ ist mit einer Bestandsaufnahme im 
Vorfeld des Starts einer Kampagne vergleichbar, an die dann die weitere SocialMedia-Analyse anschließt. Im Rahmen einer Social-Media-Analyse, die nach dem Start der Kampagne durchgeführt wird, werde die Messung zu mehreren Zeitpunkten wiederholt. Somit haben sowohl die Definition der Kampagnenziele als auch die Durchführung der „Nullmessung“ Einfluss auf die Festsetzung von im Marketing üblichen KPIs als zu messende Faktoren, die den einzelnen SocialMedia-Analysen zugrunde liegen und auch als deren „Grundpfeiler“ bezeichnet werden. Im Zusammenhang der Festsetzung von KPIs wird außerdem bestimmt, welche Social-Media-Plattformen, Wettbewerber, Märkte und Zielgruppen im Rahmen der Social-Media-Analysen zu betrachten sind und anhand welcher Suchbegriffe dies geschehen soll. Die von Social-Media-Agenturen durchgeführten Untersuchungen, die nicht der Messung von Kampagnen dienen, sondern die Social-Media-Kanäle allgemein „,beobachten“ oder aber Sonderanalysen darstellen, unterscheiden sich bezüglich des Prozesses ihrer Umsetzung von der eben geschilderten kaum. Im Hinblick auf Listening ist zu ergänzen, dass dieses laut den Praktikern themenspezifisch erfolgt und nicht anhand eines festgesetzten Schemas.

Social-Media-Analysen setzen die Marketing-Abteilungen vor allem mit quantitativem Fokus um. Es würden automatisierte Frequenzanalysen zur Ermittlung der Häufigkeit der Nennung von bestimmten Begriffen, Produkten oder Marken in sozialen Medien durchgeführt. In diese Frequenzanalysen, die zumeist im Zuge eines regelmäßigen Monitorings stattfinden, sind auch Betrachtungen relevanter Wettbewerber integriert. Ergänzt werden die quantitativ orientierten Frequenzanalysen, die manche Praktiker als „oberflächlich“ beschreiben, oft um ,qualitativ“ genannte, teilweise manuell umgesetzte Tonalitätsanalysen. Ein Befragter merkt an, dass einer quantitativen Analyse alleine kaum Aussagekraft zukomme. Eine verstärkte Integration, qualitativer' Analysen sei notwendig, da nur diese Kampagnenerfolge erklären könnten: 
Befragter: Also ic/ wir neigen dazu eher quantitativere zu machen im Marketing, obwohl es, glaube ich, eher eine Kombination aus beiden sein sollte. Ne, weil so jemand/, das interessante Beispiel, was wir auch immer nennen in der Diskussion, ist, „es bringt uns nix zu sehen, dass irgendwie 10.000 Leute in England im letzten Monat über [das neue Fahrzeug Modell Rot] getweetet haben“. Punkt. Weil das vielleicht alles, irgendwie von den 10.000 waren es 8.000 Spambots, die einfach unsere Pressemitteilung ge-re-postet haben, was in der Tat oft passiert. Wenn wir hier eine Pressemitteilung rausgeben, gibt es Tweets, die, äh, Twitter Accounts, die das einfach nur duplizieren ohne eigene Reichweiten zu haben. Wenn wir jetzt nur sehen würden, okay, 8.000 Leute haben über [das Fahrzeug Modell Rot] getweetet, würden wir sagen, „okay, [das Fahrzeug Modell Rot] ist gerade aktuell relativ viel diskutiert“ und wir dann nicht uns anschauen würden, dass vielleicht nur fünf Leute über [das Fahrzeug Modell Blau] getweetet haben, die aber irgendwie gerade, äh, alles Twitter-Profile waren, die nochmal eine halbe Million eigene Follower mitbringen, würden wir sehen, wie fünf Leute über 3.000, also ist [das Fahrzeug Modell Rot] gerade sozusagen das aktuelle Thema, was wir, äh, im Internet sehen. Über was, äh, irgendwie gegenseitig diskutiert wird. Würden wir uns qualitativ diese fünf Profile angucken gegenüber den 3.000, würden wir relativ das umdrehen und sagen, ,irgendwie fünf Influencer mit insgesamt drei Millionen Reichweite haben über [das Fahrzeug Modell Blau] getweetet und von den 3.000 [Accounts zum Fahrzeug Modell Rot] hat es eh keiner gelesen, weil es alles nur Spambots sind“. Also von daher, wir neigen dazu eher quantitativ zu messen, sollten aber mehr eine Kombination aus Qualität und Quantität machen (I9/ S7/ Z12-30).

In Bezug auf Tonalitätsanalysen merken die Befragten jedoch an, dass diese, sofern deren Durchführung über Software erfolgt, noch erhebliche Defizite aufweisen. Daher würden die Social-Media-Agenturen deren Ergebnisse teilweise manuell überprüfen.

Ferner führen die Befragten Influencer-Analysen als einen weiteren Bestandteil der praktizierten Social-Media-Analysen an. Dabei würden quantitativ orientierte Social-Media-Analysen manuell um Originalbeiträge aus sozialen Medien ergänzt, wenn zum Beispiel Leitmedien auf die Kampagnen des Automobilherstellers reagieren. Influencer Analysen gelten den Praktikern wie auch Tonalitätsanalysen als ,qualitative" Elemente von Social-Media-Analysen. Mit diesen Verfahren gehen, so wird berichtet, nicht selten Zeitraumbetrachtungen, im Rahmen derer soziale Medien im Hinblick auf inhaltliche Veränderungen der in diesen veröffentlichten Beiträge untersucht werden, einher. So sind gegebenenfalls Rückschlüsse bezüglich der Modifikation von Einstellungen zu den in Kampagnen beworbenen Produkten festzustellen.

Nach Abschluss einer Social-Media-Analyse und damit zumeist an eine Kampagne anschließend würden deren Ergebnisse mit dem Resultat der im Vorfeld durchgeführten „Nullmessung“ verglichen. Zudem erfolgt laut den Befragten eine quantitative Prüfung unter der Fragestellung, inwiefern die Ergebnisse mit dem Einsatz der aufgewendeten Mittel korrespondieren. An den Automobilhersteller 
werden die Ergebnisse im Fall von Listening nach deren Abschluss, im Fall von kontinuierlichem Monitoring in Form regelmäßiger Berichte, zum Beispiel im monatlichen Rhythmus, durch die Social-Media-Agentur geliefert. Es folge eine unternehmensinterne Ergebnispräsentation und Weiterleitung an entsprechende Schnittstellenpartner.

Die Befragten charakterisieren das Vorgehen bei den Social-Media-Analysen, die von Social-Media-Agenturen durchgeführt werden, als sehr pragmatisch. Untersuchungen würden oft nach dem Prinzip „Trial and Error“ praktiziert. Im Rahmen der Berichte über „Nullmessungen“ vor Kommunikationsmaßnahmen ist ferner die Rede von „A/B-Tests“ im Anschluss:

Befragter: Wir haben in Facebook Insights reingeschaut und g/, äh, (stockt) wir haben A/B-Tests durchgeführt, wo, äh, die Reichweite besser oder schlechter ist. (Atmet ein) bei Posts, bei Bilder, bei Videos. Und daraus haben wir unsere Schlussfolgerungen gezogen. Als simples A/B-Testing (I17/ S4/ Z28-30).

Auch die Thematisierung von „A/B-Tests“ weist auf einen pragmatischen Umgang mit Social-Media-Analysen hin. Ein solcher zeigt sich, obwohl die mit Social-Media-Agenturen durchgeführten Social-Media-Analysen im Vergleich mit den über Desk Research umgesetzten innerhalb des untersuchten Datenmaterials als eher professionell gelten oder sogar von den Praktikern zum Zweck der Professionalisierung bei Social-Media-Agenturen beauftragt werden.

Es lässt sich festhalten, dass Social-Media-Analysen in den MarketingAbteilungen des Automobilherstellers vor allem als regelmäßiges, automatisiertes Monitoring über Tools praktiziert werden; gelegentlich findet eine Ergänzung um zumeist manuell umgesetztes Listening statt. Monitoring ordnen die Praktiker dabei als quantitative, Listening als ,qualitative" Variante von Social-MediaAnalysen ein. Ebenso wenig wie die Mehrheit der befragten Social-MediaAnalysten des Automobilherstellers erwecken die Praktiker der Abteilungen für Marketing den Eindruck, als würden sie über vertiefte Kenntnisse des sozialwissenschaftlichen Methodenkanons verfügen und reflektiert mit SocialMedia-Analysen und deren Methodik umgehen. Ihre Praxis scheint vielmehr durch Pragmatismus bestimmt zu sein und entspricht offenkundig trotzdem oder gerade deshalb den Anforderungen des Unternehmens. Etliche Erfahrungen mit diversen Arten der Social-Media-Analyse und deren Umsetzung durch mehrere Dienstleister liegen in den Marketing-Abteilungen vor. Die Praktiker dieser Abteilungen sind, wenn auch ohne vertiefte, vielleicht auch nicht benötigter Kenntnisse des sozialwissenschaftlichen Methodenkanons über ihre Praxis der Social-MediaAnalysen im Bilde und dazu in der Lage, deren Prozess anhand ihrer Erfahrungen detailliert zu beschreiben. 


\subsection{Social-Media-Analysen mit Kommunikationsagenturen}

Eine im Rahmen dieser Forschungsarbeit nicht als weitere Sonderform einzustufende, aber dennoch von den Kooperationen mit anderen Dienstleistern zu unterscheidende Art der Umsetzung stellen Social-Media-Analysen mit Koтmunikationsagenturen dar. Im Untersuchungsmaterial sind Berichte über Agentur (U) enthalten. Hierbei handelt es sich um einen Dienstleister, den der Unternehmensbereich für Kommunikation des betrachteten Unternehmens mit der Umsetzung von Medienresonanzanalysen und der Erstellung von Medienspiegeln beauftragt hat. Die Social-Media-Analysen eben dieser Kommunikationsagentur unterscheiden sich insofern von anderen im Rahmen dieser Arbeit bisher thematisierten Untersuchungsformaten als es sich dabei nicht um reine SocialMedia-Analysen handelt. Im Rahmen der Untersuchungen werden nicht nur Daten von Social-Media Plattformen betrachtet, sondern ebenso Daten, die Printmedien, audiovisuellen Medien oder auch deren Onlineangeboten entnommen sind. SocialMedia-Daten markieren lediglich einen Bestandteil des gesamten Datenkorpus einer Untersuchung. Obwohl einer solchen also Daten verschiedener Medienarten zugrunde liegen, sprechen die Befragten dennoch von „Social-Media-Analysen“. Dadurch sind auch diese Berichte in das untersuchte Datenmaterial eingeflossen, werden in dieser Arbeit aber bezüglich der praktischen Umsetzung und methodischen Durchführung in einem separaten Abschnitt behandelt. Obwohl also die Social-Media-Daten in den Untersuchungen der Kommunikationsagentur nur ein Element des Datenkorpus neben weiteren markieren, versteht die hier vorliegende Studie auch sie als eine von dem Automobilhersteller praktizierte Variante von Social-Media-Analysen.

Die Praktiker unterscheiden Medienresonanzanalysen von Medienspiegeln. Bei den Medienresonanzanalysen handle es sich um ein Listening zu Adhoc-Fragestellungen, bei den Medienspiegeln hingegen um ein Monitoring, das mitunter in sozialen Medien erfolgt. Die praktischen Anwender berichten, dass sie die Untersuchungen vor der Beauftragung der Kommunikationsagentur unternehmensintern, mit Tools umgesetzt haben, dann jedoch eine vollständige Auslagerung an den Dienstleister erfolgt ist. Mit diesem werde nun täglich kooperiert. Aufgrund einer automatisierten Datenerhebung könne die Agentur größere Datenmengen bewältigen, als es dem Unternehmen mit Tools möglich war. Ferner setze die Agentur das Monitoring professionalisiert, über 24 Stunden hinweg um und stelle zudem durchgehend Ressourcen, auch personeller Art, für die Bearbeitung eines kurzfristigen Listening bereit. Unternehmensintern seien diese Faktoren bei der Umsetzung von Social-Media-Analysen nicht gegeben. 
Vor seiner Beauftragung wurde der Dienstleister anhand von Tests ausgewählt und gemäß den Befragten individuell zu der Durchführung von Untersuchungen für ihr Unternehmen über mehrere Monate „qualifiziert“. Eine im Vorfeld der Untersuchungen hergestellte Vertrautheit der Agentur mit dem betrachteten Unternehmen sei notwendig; ein genaues Briefing sei ebenso relevant wie der persönliche Kontakt:

Befragter: [. . . ] Da muss das Briefing wirklich sitzen. Die müssen [das Emblem der Marke des Unternehmens des Befragten] in die Linse tätowiert haben. Nur dann geht es (I19/ S10/ Z44-45).

Im Vorfeld der Durchführung von Social-Media-Analysen seien mit der Agentur die zu analysierenden Medien bestimmt worden. Bei diesem Schritt, so zeigt das untersuchte Datenmaterial, wurden auch soziale Medien von Bedeutung, welche an ihrer Größe festgemacht wurde, wie Facebook oder auch Twitter, in das „Leitmedien-Set“ integriert. Im Mittelpunkt des Interesses hätten aber nicht soziale Medien, sondern internationale Leitmedien gestanden. Eine globale Abdeckung aller Märkte oder Verkaufsregionen der Produkte und Dienstleistungen des Automobilherstellers sei das wesentliche Kriterium gewesen. Bezüglich der untersuchten Themen machen die praktischen Anwender zwar keine weiteren Angaben, merken aber an, dass bei Veranstaltungen ihres Unternehmens eine Ausrichtung daran erfolgt.

Im Fall des Medienspiegels erhebe der Dienstleister die Daten automatisiert und verfüge über diese zunächst in ,unstrukturierter Form“. Pro Tag ermittle die Agentur zweimal circa 2000 Treffer, die sie auf eine E-Mail von je 60 Seiten reduziere. Dies geschehe anhand der Untersuchung der Inhalte, die über den Automobilhersteller in den Medien veröffentlicht werden. Daraus werde abgeleitet, wie dieser, auch im Vergleich mit seinen Wettbewerbern, wahrgenommen wird. Ferner werde untersucht, ob die eigenen in den Medien platzierten Botschaften verstanden oder aber negiert würden und welche Reichweite diesen zukomme. Damit einher gehe eine Influencer-Analyse. Eine solche Beschreibung lässt erneut Inhaltsanalysen vermuten; die Befragten geben diese jedoch nicht an. Untersuchungen würden Frequenzanalysen und Tonalitätsanalysen umfassen.

Zwar heißt es, dass Erfahrungen mit Social-Media-Analysen vorhanden seien und die Methode längst ein Bestandteil des Tagesgeschäfts sei, doch das genaue Vorgehen bei deren Durchführung oder der Auswertung der erhobenen Daten ist den Befragten, nach ihren eigenen Angaben, nicht bekannt. Dabei handle es sich um ein Betriebsgeheimnis der Agentur. Dies legt erneut die Vermutung von absolutem ,Agenturvertrauen“ nahe. Eingeschränkt scheint dieses ,Agenturvertrauen“ 
nur im Vorfeld der Kooperation und zu deren Beginn zu sein, da die Agentur vor ihrer Beauftragung Tests durchlaufen und Informationen in Bezug auf das Unternehmen erhalten hat. Bei den Praktikern sind keine weiteren Kenntnisse im Hinblick auf die praktische Umsetzung und methodische Durchführung der Untersuchungen vorhanden. Kenntnisse sozialwissenschaftlicher Forschungsmethoden fehlen. Lägen solche vor oder wären diese fundiert, wäre eine vertiefte Auseinandersetzung mit der angewandten Praxis und Methodik der Agentur erfolgt und es bestünde kein derart , blindes Vertrauen' in diese.

Da die notwendigen Lizenzen für den Medienspiegel mit hohen Kosten verbunden sind, werden dessen Ergebnisse von den Befragten lediglich an einen sehr eingeschränkten Personenkreis weitergegeben, dem neben hierarchisch in der Organisationsstruktur des Unternehmens hoch angesiedelten Personen nur seine Kommunikatoren angehören. Andere Interessierte seien in einem E-Mail-Verteiler organisiert und würden zweimal täglich eine E-Mail mit einem aus drei Meldungen bestehenden Kurztext erhalten. Die Ergebnisse der Untersuchungen werden oft nicht weiterverwendet oder kaum genutzt. Der Kommunikationsbereich bewertet die Bestandteile von sozialen Medien in seinen Untersuchungen als nicht sehr wertvoll.

Medienresonanzanalysen beschreiben die Befragten als „Abbildungen von Themen", die ihr Unternehmen betreffen. Fragestellungen müssten meist kurzfristig beantwortet werden, so dass die Untersuchungen entsprechend beauftragt würden. Die Umsetzung der Medienresonanzanalysen wird zwar genau dargestellt, aber nicht derart, dass den Praktikern fundierte Kenntnisse des sozialwissenschaftlichen Methodenkanons attestiert werden könnten. Zunächst erhebe der Dienstleister einen „Social Media Buzz“, ermittelt also das Beitragsaufkommen zu vorgegebenen Themen. Social-Media-Kanäle würden durchgesehen, relevante Beiträge entnommen, in eine E-Mail eingefügt und verlinkt. Ferner werden aus einem kleinen, festgesetzten ,Leitmedien-Set“ Inhalte einen Monat lang gesammelt, dann frequenzanalytisch unter Einbezug des Wettbewerbs ausgewertet und schließlich manuellen Tonalitätsanalysen unterzogen. Bei Medienresonanzanalysen, die auch „Listening“ genannt werden, erfolge die Erhebung der Daten automatisiert, deren Auswertung manuell:

Befragter: [... ] Die haben da ihre Suchroutinen, die sie dann weiter qualifizieren. Aber da ist einfach auch sehr viel menschliches Hirn dabei. Auch weil ich das so beauftragt habe. Weil ich eben nicht die 2000 Treffer sehen möchte oder dann unqualifiziert irgendwas hingeschmissen bekommen will, sondern auch verlange, dass sie dann, wenn sie was finden, entsprechend weiter recherchieren und sich dann quasi durchhangeln durch das Netz. Wer hat das gepostet und was macht der sonst noch und was muss ich wissen. Da wirklich intelligent durchgehen (I19/ S9/ Z24-30). 
Ergebnisse von Medienresonanzanalysen werden bestimmten Empfängern präsentiert sowie in den Managementkreisen, für die diese Ergebnisse von Bedeutung sind.

Im Hinblick auf die praktische Durchführung und methodische Umsetzung von Social-Media-Analysen mit der Kommunikationsagentur ist festzuhalten, dass es sich um Untersuchungen handelt, deren Datenkorpus aus sozialen Medien und anderen Medien gewonnen wird. Zwei Formate setzt der Dienstleister für den Automobilhersteller um: Medienresonanzanalysen und Medienspiegel. Erstere werden als Monitoring, letztere als Listening zu Ad-hoc-Fragestellungen beschrieben. Bezüglich der befragten Social-Media-Analysten des für Kommunikation zuständigen Unternehmensbereichs ist zu konstatieren: Sie besitzen selbst offenkundig keine tiefgehenden Kenntnisse des sozialwissenschaftlichen Methodenkanons, bringen ihrem Dienstleister aber trotzdem oder gerade deswegen , blindes Vertrauen“ entgegen. Das untersuchte Datenmaterial erweckt den Eindruck, als ginge nicht zuletzt mit diesem absoluten ,Agenturvertrauen ‘ die Einschätzung oder Bewertung der praktischen Durchführung und methodischen Umsetzung von Social-Media-Analysen mit Agentur (U) als professionalisiert einher.

\subsubsection{Zwischenfazit: Kompetenzmangel und,blindes Agenturvertrauen'}

Wie anhand des Untersuchungsmaterials dargelegt, praktiziert der betrachtete deutsche Automobilhersteller Social-Media-Analysen entweder in der Variante des Social Media Monitoring oder des Social Media Listening. Beide Varianten sind in den Berichten der befragten Praktiker nicht immer eindeutig voneinander zu unterscheiden. Jedoch haben beide gemeinsam, dass es bei ihrem Einsatz durchweg um die Beantwortung von Fragestellungen, die Ermittlung von Detailinformationen, die Genese von Verständnis oder die Herstellung von Orientierung geht. Untersuchungen können sowohl quantitativ als auch ,qualitativ“ konzipiert sein, wobei Monitoring eher auf quantitative, Listening hingegen auf , qualitative " Ergebnisse zielt. Die praktischen Anwender des betrachteten Unternehmens haben bereits Erfahrungen mit Desk Research, unterschiedlichen Tools und diversen Agenturen gesammelt; des Weiteren haben sie Social-Media-Analysen automatisiert und manuell durchgeführt. Sie verfügen also über umfangreiches Wissen zu der Methode der Social-Media-Analysen. Trotzdem geht aus ihren Ausführungen Kompetenzmangel und, blindes Agenturvertrauen' hervor. In Bezug auf die angewandte Praxis von Social-Media-Analysen ist folgender Befund als Zwischenfazit festzuhalten: Den praktischen Anwendern fehlt es an der erforderlichen Methodenkompetenz; sowohl in der Sozialforschung als auch in der Informatik. 
Social-Media-Analysen werden bei dem deutschen Automobilhersteller weder im Sinne der Digital Humanities noch der Computational Social Sciences praktiziert.

Desk Research setzen die Praktiker manuell und nicht standardisiert, sondern auf eine pragmatische Weise um; weder erfolgt eine methodische Systematisierung noch findet eine Reflexion statt. Tools bewerten sie als professioneller, da es sich dabei, ihrem Verständnis nach, um eine standardisierte Variante von SocialMedia-Analysen handelt. Im Gegensatz zu Desk Research, die unterschiedlich betrieben wird, kommen Tools in erster Linie für automatisierte Frequenzanalysen, Tonalitätsanalysen, Inhaltsanalysen, Videoanalysen und Influencer-Analysen zum Einsatz. Es wird sogar beabsichtigt damit die Zukunft vorherzusagen. Die hinter den Tools stehende Methodik hinterfragen ihre praktischen Anwender, die meist dem Marketing-Bereich angehören, nicht.

Unternehmensinterne Social-Media-Analysten, die mit Agenturen zusammenarbeiten, bringen diesen Dienstleistern ein nahezu ,blindes Agenturvertrauen ‘ entgegen. Gemeint ist, dass der Automobilhersteller die angewandte Praxis und Methodik der Dienstleister, die mit der methodischen Umsetzung und praktischen Durchführung von Social-Media-Analysen beauftragt wurden, nicht hinterfragt. Sind Agenturen erst einmal beauftragt, so wird diesen nahezu , blind vertraut'. Zum einen mag dies darin begründet sein, dass sich Dienstleister dem Unternehmen gegenüber als Experten für Social-Media-Analysen präsentieren und von den Praktikern des Unternehmens auch in eben der Expertenrolle, die sie sich selbst zuschreiben, wahrgenommen werden. Zum anderen offenbart das Untersuchungsmaterial, dass es den Social-Media-Analysten des Automobilherstellers an fundierten Kenntnissen des sozialwissenschaftlichen Methodenkanons fehlt. Da es an Methodenwissen im Bereich der Sozialforschung mangelt, ist anzunehmen, dass die Befragten gar nicht dazu imstande sind, die angewandte Praxis und Methodik der Dienstleister zu hinterfragen. Sie sind sich dieses Mangels an sozialwissenschaftlicher Methodenkompetenz teilweise offenkundig bewusst und versuchen sich daher erst gar nicht an methodischer Reflexion. Dies erklärt auch, dass dem dieser Arbeit zugrunde liegenden Untersuchungsmaterial nur oberflächliche Ausführungen in Bezug auf die angewandte Praxis von Social-Media-Analysen und die dahinterstehende Methodik zu entnehmen sind. Ein Mangel an ,Informatikkompetenz' kommt hinzu. Dies bedeutet, dass die praktischen Anwender ebenso wenig die hinter gewissen Arten der Social-Media-Analyse stehende Methodik der Informatik verstehen oder verstehen können und daher auch nicht darzulegen vermögen. Etliche Befragte streichen auf der einen Seite Software, Tools, Crawler oder Dashboards als besondere Merkmale von Social-Media-Analysen heraus; auf der anderen Seite betonen sie aber auch, dass „menschliche“ Analysten die Social-Media-Analysen umsetzen und durch manuelle Codierung realisieren. So war Folgendes festzustellen: Die befragten Social-MediaAnalysten des Automobilherstellers verfügen weder über fundierte Kompetenz in der 
Informatik noch in der Sozialforschung. Sie selbst sind nicht dazu in der Lage, Software, Tools, Crawler oder Dashboards zu verstehen oder zu erklären, geschweige denn zu programmieren. Ferner scheinen sie selbst noch nie codiert, also Daten anhand von Codierverfahren ausgewertet zu haben. Zwar wenden sie die Methode der Social-Media-Analysen praktisch an, doch ihr Vorgehen beruht auf methodischem Unwissen. In diesem Unwissen liegt auch die Scheu der Befragten begründet, ihre angewandte Praxis und die Methodik von Social-Media-Analysen generell zu hinterfragen. Eine kritische Reflexion ist bei den Praktikern des Unternehmens nicht zu verzeichnen.

Es fehlt also an ,Informatik- und Sozialforschungskompetenz " und doch praktizieren die Befragten Social-Media-Analysen für ihr Unternehmen. Ihre Umsetzung besteht vor allem darin, dass sie Agenturen mit der Durchführung von Social-Media-Analysen beauftragen und die Dienstleister ,steuern“. Das untersuchte Datenmaterial hat einen pragmatischen, keineswegs einen professionellen Umgang offenbart, den die Social-Media-Analysten des Automobilherstellers mit der Methode pflegen. Damit werden sie ihr jedoch nicht gerecht. Die angewandte Praxis ist in allen ihren Facetten in Routine übergegangen. SocialMedia-Analysen werden so durchgeführt, wie es sich mit der Zeit etabliert hat. Kritische Reflexion war wohl nie vorgesehen. Die Social-Media-Analysen sind geprägt von Kompetenzmangel und ,blindem Agenturvertrauen'. Trotzdem gilt es zu bedenken, dass sich die Mehrheit der Social-Media-Analysten in ihren Ausführungen über die angewandte Praxis der Methode der Fachtermini der Sozialforschung bedient. Zwar mangelt es ihnen an dem für eine fundierte Umsetzung von Social-Media-Analysen notwendigen Methodenwissen, es ist jedoch nicht davon auszugehen, dass ein solches in dem Unternehmen überhaupt nicht vorhanden ist oder war. Vielmehr scheint es so, als hätten die Praktiker ihr Methodenwissen, vergessen“. Sozialwissenschaftliche Begriffe kursieren in ihren Berichten und werden verwendet, so die Annahme, da sie entweder einmal mit Inhalten verknüpft waren oder im Austausch mit anderen gefallen sind und unreflektiert übernommen wurden. Beide Optionen erklären, weshalb die Fachtermini zwar genutzt werden, ihr Gebrauch aber nicht korrekt erfolgt und eine methodische Reflexion ausbleibt. Bei Nachfragen in den Interviews zu methodischen Details, wie Codierverfahren oder auch Inhaltsanalysen, haben die Praktiker keine Erklärungen geboten, sondern Ausflüchte gesucht. Manche Befragte scheinen zwar sozialwissenschaftliche Methodenkenntnisse zu besitzen, doch diese sind nicht oder nicht mehr fundiert. Wegen des falschen Gebrauchs der Fachtermini ist $\mathrm{zu}$ vermuten, dass das Methodenwissen, das einmal vorhanden war, in , Vergessenheit" geraten ist. Ein Grund dieses , Vergessens “ mag darin liegen, dass es einfach nicht genutzt wird. Worin der Nichtgebrauch des Methodenwissens und damit die Ursache für sein ,Vergessen“ gründet, ist im Folgenden zu klären. 


\subsection{Social-Media-Analysen als Methode}

Das betrachtete Unternehmen der Automobilindustrie setzt Social-MediaAnalysen als Methode für die Genese von Wissen ein. Jede Forschungsmethode zeichnet sich durch eigene Charakteristika aus und birgt ihr inhärente spezifische Vor- und Nachteile. Im vorliegenden Abschnitt werden die besonderen Eigenschaften der Methode thematisiert, wie die Social-Media-Analysten des deutschen Automobilherstellers sie aufgrund ihrer praktischen Anwendung wahrnehmen. Eine Differenzierung zwischen verschiedenen Arten der Methode ist nicht möglich, da eine solche im untersuchten Datenmaterial nicht explizit auszumachen war. Zunächst werden Social-Media-Analysen als Methode zur Wissensgenese vorgestellt, indem ihre Charakteristika beschrieben werden. Daran schließt eine Thematisierung der den Social-Media-Analysen inhärenten methodischen Vorund Nachteile an.

Mit der Thematisierung von Social-Media-Analysen als Methode wird nicht zuletzt ein Beitrag zur Diskussion sozialwissenschaftlicher Forschungsmethoden geleistet. Dieser reiht sich ein in deren Modifikation und Erweiterung im Kontext des digitalen Wandels und der Untersuchung sozialer Medien, also in die Reihen der Online-Forschung. Damit gehen, wie zuvor bereits gezeigt, sowohl Chancen als auch Risiken einher. Wie generell von der Sozialforschung in Bezug auf den Umgang mit dem Methodenrepertoire, das für empirische Untersuchungen bereitsteht, empfohlen, gilt es, auch der Methode der Social-Media-Analysen mit der Fähigkeit und vor allem der Bereitschaft zur kritischen Reflexion zu begegnen. Einerseits sind zwar die Potentiale der Methode zu ergreifen, andererseits ist es aber unabdingbar, ihre Herausforderungen zu erkennen. ${ }^{8}$

\footnotetext{
${ }^{8}$ Zwei Aspekte, die es im Verlauf dieses Abschnitts zu beachten gilt, sind anzusprechen. Zum einen geht es nicht um die Frage, aus welchen Gründen Social-Media-Analysen als Methode eingesetzt werden; diese werden im Rahmen der Diskussion der Typen der Wissensverwendung dargelegt. Zum anderen beruhen sowohl die Charakteristika von Social-Media-Analysen als auch ihre Vor- und Nachteile auf dem für Studie untersuchten Datenmaterial. Die Darstellung gründet auf den praktischen Erfahrungen, die die befragten Praktiker bei der Anwendung gemacht haben. Im Hinblick auf Verallgemeinerungen bezüglich der Methode der Social-Media-Analysen ist dies zu bedenken. Um allgemeingültige Aussagen in Bezug auf Social-Media-Analysen treffen zu können, empfiehlt es sich, die Untersuchung, auf der diese Arbeit fußt, in anderen Forschungsfeldern zu wiederholen und um weitere Daten zu ergänzen.
} 


\subsubsection{Charakteristika der Methode}

Sozialwissenschaftliche Forschungsmethoden, sowohl qualitativer als auch quantitativer Art, sind durch je eigene, ihnen inhärente Eigenschaften charakterisiert, aufgrund derer die Methoden dann auch als für verschiedene Fragestellungen oder Forschungsvorhaben geeignet beziehungsweise angemessen eingeschätzt und zum Einsatz gebracht werden. Charakteristika der Methode, die die befragten Social-Media-Analysten ihr beimessen, behandelt der vorliegende Abschnitt. ${ }^{9}$

Im untersuchten Datenmaterial werden Social-Media-Analysen als Verfahren für die Untersuchung der in sozialen Medien enthaltenen Stimmungen dargestellt und oftmals direkt, manchmal auch nur indirekt als Methode der Meinungsforschung eingeordnet. Meinungsforschung, auch als Demoskopie bekannt, beschreibt Karl-Heinz Hillmann (2007) als Umfrageforschung, die auf statistischen, psychologischen und auch empirisch-soziologischen Methoden beruht. Ihre Verfahren würden dazu dienen, soziale Massenerscheinungen zu „beobachten“ beziehungsweise $\mathrm{zu}$ analysieren: ${ }^{10}$

M[einungsforschung] ermittelt Meinungen als Antworten auf gezielte Fragen, um damit Aufklärung über Wertorientierungen, Einstellungen und voraussichtliches Verhalten geben zu können. Damit wird allg[emein] oder bei bestimmten Personenkategorien übliches Verhalten diagnostiziert, um Aussagen über Regelmäßigkeiten sozialen Handelns und Reagierens oder über die Wirkungen von sozialen Einflussfaktoren oder Medien zu ermöglichen. In diesem Zusammenhang ist M[einungsforschung] sowohl als empir[isch]-induktives Vorstadium für die Entwicklung von soziolog[ischen] Hypothesen wie als Überprüfungs-Instrument soziolog[ischer] Theorien zu betrachten (Hillmann, 2007, S. 554).

Zu diesem Zweck würde die Meinungsforschung, so Hillmann weiter, die Methoden der empirischen Sozialforschung nutzen und vorrangig Befragungen und

\footnotetext{
${ }^{9}$ Die Methoden empirischer Sozialforschung thematisieren mitunter: Bohnsack, 2014; Diekmann, 2016; Döring, \& Bortz, 2016; Flick, Kardorff, \& Steinke, 2012; Flick, Kardorff, Keupp, Rosenstiel, \& Wolff, 1995; Flick, 2011, 2009; Kromrey, Roose, \& Strübing, 2016; Lamnek, 2016; Rosenthal, 2014; Schnell, Hill, \& Esser, 2018.

${ }^{10} \mathrm{Zu}$ Meinungsforschung liegen etliche Arbeiten vor. Noelle-Neumann (1980/2002), die als Pionierin der Demoskopie in Deutschland gilt, hat in Bezug auf die öffentliche Meinung ihre berühmte, auch heute noch vielbeachtete Theorie der Schweigespirale formuliert. Siehe hierzu zum Beispiel: Noelle-Neumann, 1980/2001, 2009, 1992; Roessing, 2007, 2011; Schulz, \& Rössler, 2013. Die verschiedenen Methoden der Meinungsforschung werden beispielsweise thematisiert in: Engel, 2015; Jacob, Heinz, Décieux, 2013; Noelle-Neumann 2005.
} 
Beobachtungen einsetzen. Als Hauptprobleme der Meinungsforschung erkennt Hillmann

[... ] die möglichst objektive (von subjektiven Verzerrungen der Erhebungspersonen freie) Sammlung von Daten, die Übersetzung von Problemstellungen in zahlenmäßig greifbare Merkmale und Eigenschaften, d. h. die Übersetzung von Begriffen, Vorstellungen, Einstellungen, Ideen in „Dimensionen“ oder „Indikatoren“, sowie die analytische Verarbeitung und Interpretation der ermittelten und quantifizierten Daten (Hillmann, 2007, S. 554f.).

Marktforschung wiederum benennt Hillmann als das wichtigste Anwendungsgebiet der Meinungsforschung. Es ist nicht davon auszugehen, dass den für diese Forschungsarbeit Befragten diese oder eine andere wissenschaftliche Definition von Meinungsforschung bekannt ist. Mit ihrer Beschreibung von Social-MediaAnalysen als Meinungsforschung und der entsprechenden Bezeichnung versuchen sie den Untersuchungsgegenstand von Monitoring und Listening zu umreißen. Sie gehen davon aus, dass es insbesondere , subjektive" Meinungen, Anliegen, Bedürfnisse und Wünsche der Nutzer sozialer Medien, aber auch Kunden- oder Nutzermotive sowie deren Dispositionen sind, die als Botschaften in SocialMedia-Kanälen platziert werden. Laut Hillmann beabsichtigt es die Meinungsforschung und damit gewissermaßen auch die Methode der Social-Media-Analysen, die ,subjektiven“ Meinungen, Anliegen, Bedürfnisse und Wünsche der Nutzer sozialer Medien sowie Kunden- oder Nutzermotive und deren Dispositionen zu ,objektivieren" und letztlich zu quantifizieren. Mit den durch Monitoring und Listening verfolgten Bemühungen des Quantifizierens von in Social Media enthaltenen Inhalten werden die Verfahren gemäß den Befragten den Erwartungen ihres Unternehmens gerecht und stehen somit in Einklang mit dessen Denkstil oder ,Logik'.11

Zudem spannen die Praktiker im Hinblick auf die Charakterisierung von Social-Media-Analysen als Methode ein Kontinuum zwischen ,Subjektivität“ und ,Objektivität' auf. Es ist die Rede davon, dass sie über Verfahren wie Monitoring und Listening die , subjektiven“ Meinungen, Anliegen, Bedürfnisse und Wünsche der Nutzer sozialer Medien sowie Kunden- und Nutzermotive und deren Dispositionen erheben und vor allem ,objektivieren“. ,Hinter“ jeder in sozialen Medien platzierten Botschaft stecke zunächst eine, subjektive“ Aussage, da diese durch Subjekte formuliert worden sei:

${ }^{11}$ Ein weiterer Grund dafür, dass die Praktiker Social-Media-Analysen in einen Zusammenhang mit der Marktforschung stellen, besteht wohl darin, dass die hier vorliegende Forschungsarbeit durch die für Marktforschung zuständige Abteilung des betrachteten Unternehmens gefördert wurde. Der Feldzugang beruhte auf der temporären Zugehörigkeit der Verfasserin dieser Arbeit zu der Abteilung. Bereits beim Erstkontakt wurde dies den Befragten offengelegt. Damit war ersichtlich, dass die unternehmenseigene Abteilung für Marktforschung über die Ergebnisse dieser Studie unterrichtet wird. 
Befragter: [... ] Weil ja immer noch der (stockt) Mensch mit seiner Motivation dahinter steckt (I15/ S2/ Z1-2).

Teilweise werden unter dem Aspekt der ,Subjektivität 'Social-Media-Analysen auch als ,qualitative' Methode per se dargestellt. Im Vergleich mit quantitativen Verfahren seien qualitative weniger, objektiv'. Jedoch räumen die Befragten auf der der ,Subjektivität' entgegengesetzten Seite des Kontinuums durchaus ein, dass Social-Media-Analysen dazu beitragen, ,Objektivität ' in ihr Unternehmen ,hinein zu transportieren“. Monitoring wie Listening würden diesem einen „Spiegel vorhalten" und dadurch einen bedeutenden Beitrag zu dessen Selbstreflexion und der Etablierung von ,Objektivität", innerhalb seiner Mauern' leisten. Durch die über die Methode erzielte ,Rationalität" würden ,subjektive Sichtweisen objektiviert", zum einen die der Nutzer sozialer Medien, zum anderen die des untersuchten Unternehmens selbst:

Befragter: (...) Mhm (überlegend), naja, dass man eine rationalere, äh, objektivere Sicht (stockt) auf die/ auf die Posts kriegt, auf die Fan-Gruppe, auf, ähm/. Vielleicht hat man da manchmal ein bisschen, ähm, eine rosa Brille darauf und denkt sich, das muss doch funktionieren, tut es aber überhaupt nicht. Einfach eine rationalere Sicht auf die Dinge (I24/ S4/ Z5-8).

Ein weiteres Charakteristikum der Methode ist es laut den Social-MediaAnalysten, dass Monitoring und Listening Extrema beziehungsweise Extrempositionen liefern. Die in sozialen Medien platzierten Botschaften seien keineswegs mit dem Durchschnitt der in einer Gesellschaft vorzufindenden Meinungen gleichzusetzen. Nach Ansicht der Befragten könnten keine in Bezug auf die Gesamtgesellschaft repräsentativen Aussagen über Social-Media-Analysen gewonnen werden. Fragestellungen, die der gesamten Gesellschaft als Grundgesamtheit bedürfen, seien weder über Monitoring noch über Listening zu beantworten. Durch Social-Media-Analysen könnten stattdessen extreme Meinungen, sowohl sehr positive als auch sehr negative, erhoben werden. Nach Ansicht der SocialMedia-Analysten eignet sich die Methode daher nicht dazu, die Relevanz, Bedeutung oder Gewichtung, die einem Thema zukommt, zu ermitteln. Ihre Ergebnisse könnten nicht als Basis für Rückschlüsse auf eine große Grundgesamtheit herangezogen werden. Über Monitoring und Listening erzielte Ergebnisse seien als Erkenntnisse, die auf Extrempositionen und Einzelfällen beruhen, einzuordnen und auch zu behandeln.

Social-Media-Analysen sind des Weiteren nicht in jeder Hinsicht planbar. Es seien viele Faktoren, die auf eine Untersuchung mit dieser Methode einwirken und damit die Güte deren Ergebnisse beeinflussen können. So schätzen die befragten 
Praktiker Monitoring und Listening zwar nicht als geeignete Basis für strategische Ableitungen ein, doch sie sprechen den Verfahren andere Fähigkeiten zu, etwa das Aufdecken von Tendenzen und Ableiten von Trends.

Wie bereits erwähnt, unterscheiden die Befragten mitunter zwischen quantitativen und ,qualitativen" Verfahren und stellen Social-Media-Analysen bald als eher quantitative, bald als eher, qualitative" Methode dar. Ebenso wird in den Ausführungen über das Vorgehen bei der Datenauswertung bisweilen zwischen quantitativen und ,qualitativen" Inhaltsanalysen differenziert, diese werden mit einer Ausnahme jedoch nie entsprechend benannt. Wird das Vorgehen ähnlich einer quantitativen Inhaltsanalyse geschildert, geht dies oft mit der Darstellung und Bezeichnung als „Monitoring“ oder ,,automatisierte“ Social-Media-Analyse einher. Greift man in der Auseinandersetzung mit diesen Schilderungen auf sozialwissenschaftliche Methodenkenntnisse zurück, ist bei mancher Ausführung zu Listening jedoch eine Nähe zu qualitativen Inhaltsanalysen festzustellen, vor allem wegen des manuellen Vorgehens, von dem berichtet wird. Den Ausführungen der Befragten ist jedoch keine etablierte Struktur zu entnehmen, die den diversen Varianten von Social-Media-Analysen zugrunde liegt. Eine solche kann aus dem untersuchten Datenmaterial auch nicht rekonstruiert werden, da es an eindeutigen Querverbindungen zwischen den einzelnen Unterscheidungen, die angestellt werden, mangelt. Es ist festzuhalten, dass die folgenden Gegensatzpaare im Hinblick auf die Methode der Social-Media-Analysen gebildet werden: Monitoring versus Listening, automatisiertes versus manuelles Vorgehen, quantitative versus ,qualitative" Verfahren, quantitative versus ,qualitative“ Inhaltsanalyse. Anhand des Untersuchungsmaterials zeichnet sich außerdem ab, dass die befragten Social-Media-Analysten ebenso wenig wie die von ihnen genannten Dienstleister nicht dazu in der Lage sind, eine auf solchen Vergleichen beruhende Struktur in Bezug auf die Methode aufzuspannen. Die Annahme der nicht vorhandenen Fähigkeit, sowohl bei den befragten Praktikern als auch bei den von ihnen beauftragten Agenturen, beruht auf einem im untersuchten Datenmaterial deutlich in Erscheinung getretenen Mangel an Expertise in sozialwissenschaftlichen Forschungsmethoden.

Die Social-Media-Analysten mahnen des Weiteren eine realistische Einschätzung der Methode an. Eine Umsetzung von Social-Media-Analysen erfolge im Vergleich mit anderen Verfahren, die zum Beispiel der Marktforschung zugerechnet werden, zwar schnell, nehme aber doch eine gewisse Zeit in Anspruch. Ebenso seien die für eine Durchführung von Monitoring und Listening erforderlichen Ressourcen wie Geld und personelle Kapazität geringer, müssten aber trotzdem aufgebracht werden. Ferner sei für die Durchführung von Monitoring und Listening eine gewisse Fehlertoleranz nötig. 
Gewisse Charakteristika, die anhand des Untersuchungsmaterials als für die Methode typisch herausgearbeitet werden, sind auf besondere Spezifika sozialer Medien, denen die über Monitoring und Listening analysierten Daten entstammen, zurückzuführen. In diesem Sinne merken die Befragten an, dass die Nutzer von Social Media sehr heterogen und zudem nicht unbedingt Kunden ihres Unternehmens sind. Aus diesem Grund würden Social-Media-Analysen keine Gewähr dafür bieten, dass sich deren Ergebnisse zum einen auf die eigene Zielgruppe beziehen und dass damit zum anderen Fragestellungen bezüglich der Kunden beantwortet werden können. Ferner vermuten die praktischen Anwender eine Passivität der eigenen Kunden in sozialen Medien. Sie gehen davon aus, dass ihre Kunden Social-Media-Kanäle zwar passiv verfolgen, sich aber nicht darin äußern. Zudem würden Monitoring und Listening, mitunter aufgrund der Heterogenität sozialer Medien, jedoch auch im Hinblick auf ihre Geschwindigkeit und damit im Kontrast zu den langen Produktlebenszyklen in der Automobilindustrie eher Zeitpunktanalysen realisieren können als dass die Verfahren für die Betrachtung von Zeiträumen geeignet sind.

Als weiteres Merkmal sozialer Medien machen die Befragten den mit sozialen Medien einhergehenden Negativismus aus. Die Annahme der Praktiker steht dabei in Einklang mit der kommunikationswissenschaftlichen Nachrichtenwerttheorie, die Negativismus oder auch Schaden als entscheidenden Nachrichtenwert anführt. ${ }^{12}$ So stellen auch die Praktiker von Social-Media-Analysen fest, dass in sozialen Medien kein Gleichgewicht in Bezug auf positive und negative Inhalte herrscht, sondern dass der Negativismus in ihnen überwiegt. Bei Social-MediaAnalysen sei daher immer zu bedenken, dass Nutzer sozialer Medien eher dazu tendieren, ihren Unmut kundzutun, als dass sie positive Rückmeldungen zu Produkten des betrachteten Unternehmens geben.

Mit sozialen Medien gehe für die Automobilindustrie ferner eine Art Kontrollverlust in Bezug auf die eigenen Marken einher. Inhalte sozialer Medien seien weniger steuerbar als die der etablierten klassischen Medien, mit welchen zahlreiche, noch bestehende Kooperationen geschlossen wurden, so dass auf gefestigte Beziehungen zurückgegriffen und ein respektvoller Umgang miteinander gepflegt wird. Diese Ansicht rührt mitunter daher, dass mit sozialen Medien einige Befragte die Gefahr eines Shitstorms verbunden sehen. Das Risiko eines solchen sei sowohl bei dem aktiven „Betreten“ sozialer Medien von Seiten des Unternehmens als auch bei von ihm selbst durchgeführten Social-Media-Analysen zu bedenken. ${ }^{13}$

12 Siehe hierzu beispielsweise: Eilders, 1997; Galtung, \& Ruge, 1965; Kepplinger, 1998; Schulz, 2009.

${ }^{13}$ Bei einem Shitstorm handelt es sich um eine massenhaft in digitalen oder sozialen Medien geäußerte Entrüstung. Ein Shitstorm richtet sich gegen Individuen, Gruppen oder aber Organisationen, greift deren Reputation an und geht teilweise mit beleidigenden Äußerungen einher. Siehe hierzu: Prinzing, 2012. 
Wie das angesprochene Risiko eines Shitstorms oder der erwähnte, mit sozialen Medien assoziierte Negativismus zeigen, werden, so eine weitere von den Praktikern geäußerte Annahme, in Social Media nicht selten zielgerichtete Botschaften platziert. Die Nutzer sozialer Medien und Kunden ihres Unternehmens, so meinen Befragte, würden teilweise auch die Absicht verfolgen, mit ihren Botschaften den Automobilhersteller zu erreichen. Es sei zu bedenken, dass die in Social-Media-Analysen untersuchten Inhalte mit einer bestimmten Absicht oder gewissen Hintergedanken von den Nutzern platziert wurden. Auch wenn die Social-Media-Analysten dies nicht direkt empfehlen, sollte mit den via Monitoring und Listening generierten Ergebnissen ein reflektierter Umgang gepflegt werden.

Die Inhalte sozialer Medien werden also von ihren Nutzern bestimmt. Dies machen die Befragten als ein Spezifikum von Social-Media-Plattformen aus. Aufgrund dessen stellen sie aber auch fest, dass in sozialen Medien nicht unbedingt Diskussionen zu jedem Thema stattfinden, an dem ihr Unternehmen Interesse hat und zu dem es eine Social-Media-Analyse durchführen möchte. Die in diesen platzierten Botschaften seien von Seiten ihres Unternehmens ebenso wenig steuerbar wie die darin geführten Diskussionen. Im Vorfeld der Umsetzung von Monitoring und Listening sei es daher notwendig zu klären, ob im Hinblick auf die Fragestellung, der nachgegangen werden soll, überhaupt Inhalte in sozialen Medien vorhanden und auch zugänglich sind. Dass es dem eigenen Unternehmen nicht direkt und unmittelbar möglich ist, Einfluss auf die Themen in sozialen Medien zu nehmen, werten die praktischen Anwender einerseits als Verlust der Hoheit des Agenda Settings, also des Festsetzens von in Verbindung mit der deutschen Automobilindustrie diskutierten Themen, andererseits als Kontrollverlust hinsichtlich der eigenen Marke. ${ }^{14} 15$

\footnotetext{
${ }^{14}$ Als ,Agenda Setting“ bezeichnet die Kommunikationswissenschaft die Thematisierungsfunktion der Massenmedien. Siehe hierzu: Bulkow, \& Schweiger, 2013; Jäckel, 2011, S. 189 ff.; Kepplinger, 2009; McCombs, \& Shaw, 1972, 1993; Rössler, 1997; Scheufele, \& Tewksbury, 2007; Weaver, 2007.

${ }^{15}$ Diese Thematik wäre im Kontext der soziologischen Kontrastierung von Macht und Herrschaft und im Rahmen einer Auseinandersetzung mit der sogenannten ,Netzöffentlichkeit“ zu beleuchten. Es wird davon ausgegangen, dass das betrachtete Unternehmen sozialen Medien eine neue Art von Macht zuspricht, die sich durch ihren digitalen Charakter auszeichnet und eine gemäß der Wahrnehmung der Befragten in der Vergangenheit etablierte Herrschaft der Automobilindustrie in Frage stellt. Diskutiert werden könnte dies unter den Stichworten „,digitale Öffentlichkeit“ oder auch ,,digitale öffentliche Meinung“. Ein Beitrag dazu kann aufgrund einer erforderlichen thematischen Begrenzung in dieser Arbeit nicht geleistet werden und muss an anderer Stelle folgen.
} 
Aufgrund der genannten Aspekte in Bezug auf Monitoring und Listening und soziale Medien im Allgemeinen sowie der Vor- und Nachteile von Social-MediaAnalysen, die im Folgenden noch dargestellt werden, zeigen die Ausführungen der Praktiker eine Ambivalenz zwischen dem Verkennen der Methode und deren Anerkennung. So wird beispielsweise eine im Vergleich zu anderen Ländern oder auch Märkten langsame Durchsetzung sozialer Medien in Deutschland diskutiert. Ergänzt wird die Diskussion um ihre Durchsetzung noch dadurch, dass diese in einem in Deutschland ansässigen Großkonzern, dem alleine wegen seiner Organisationsform und -kultur bereits eine ganz eigene Trägheit zu eigen ist, noch einmal wesentlich langsamer erfolgt ist als außerhalb:

Befragter: [. . . ] Also insofern, ähm, das wandelt sich ganz stark und das ist einfach mittlerweile/ während es jetzt für einen Marketing nix Neues mehr ist, sondern eher ein Standardkanal wie jeder andere auch, ähm, wandelt sich das einfach Stück für Stück. Das dauert halt einfach nur ein bisschen bis sich das durchsetzt und jetzt ist Deutschland nicht unbedingt das, äh, Social Media Vor/ bei Social Media an vorderster Front unterwegs. Wenn man das mit anderen Märkten vergleicht. Insofern dauert es jetzt in einem Deutschland basierten Konzern vielleicht nochmal eine Ecke länger (I13/ S8/ Z6-12).

Monitoring sei ebenso wie Listening, als es aufkam, überhaupt nicht ernst genommen worden. Teilweise geschehe dies immer noch nicht, sogar in Unternehmensbereichen und -abteilungen, für die eine Affinität zu sozialen Medien längst selbstverständlich sein sollte, wie für Kommunikation, Marketing und Marktforschung. Zu Beginn habe der Automobilhersteller Social-Media-Analysen lediglich „belächelt“. Mitunter aufgrund der mangelnden Affinität zu sozialen Medien fehle es nach wie vor an Prozessen für den Umgang mit ihnen und auch mit den über Social-Media-Analysen generierten Erkenntnissen. Die Methode der SocialMedia-Analysen werde, so beklagen sich einige Befragte, in ihrem Unternehmen immer noch nicht richtig ernst genommen. Dies habe schon dazu geführt, dass die durch Social-Media-Analysen gewonnenen Erkenntnisse ,verpufft“ sind. Eine Ambivalenz zwischen dem Verkennen, wie eben dargestellt, und der Anerkennung von Social-Media-Analysen als Methode ist anhand des untersuchten Datenmaterials dennoch festzustellen, da darin ebenso Berichte zu verzeichnen sind, welche der eben thematisierten Einstellung gegenüber Social-Media-Analysen und der Bewertung dieser Methode geradewegs diametral entgegenstehen.

Es bleibt festzuhalten, dass Monitoring und Listening in dem betrachteten Unternehmen eine andere Wertschätzung erfahren und einen anderen Stellenwert einnehmen als in der Vergangenheit. Jedoch werden Social-Media-Analysen teilweise noch immer verkannt. Anerkannt wird die Methode umso mehr, je weiter 
sich soziale Medien etablieren. Die Ambivalenz zwischen Verkennen und Anerkennung von Social-Media-Analysen wirkt sich auf die Bewertung der Methode aus und sollte daher bei der Rezeption ihrer Vor- und Nachteile bedacht werden.

\subsubsection{Vorteile der Methode}

Neben spezifischen Charakteristika der Methode sind dem untersuchten Datenmaterial besondere methodische Vorteile zu entnehmen, die die befragten Praktiker mit Social-Media-Analysen assoziieren. Diese Vorteile der Methode behandelt dieser Abschnitt.

Bei einigen Befragten ist eine Art grundsätzliche Begeisterung und Aufgeschlossenheit für digitale Verfahren wie Monitoring und Listening festzustellen. Sie beruht meist auf positiven Erfahrungen aus der Vergangenheit. Ein wesentlicher Grund für die positive Bewertung von Social-Media-Analysen und zugleich ein Vorteil, den die praktischen Anwender in Monitoring und Listening sehen, besteht in der erhöhten Bereitschaft der Nutzer sich zu äußern, die mit sozialen Medien verbunden ist:

Befragter: Ich glaube, die Bereitschaft zu einer Aussage ist halt deutlich größer. Insbesondere eine kurze, schnelle Aussage, ein kurzes, schnelles Feedback, äh, wo man natürlich aber auch dann eben zuhören muss (I29/ S8/ Z4-6).

Die befragten Social-Media-Analysten sind der Ansicht, dass die Nutzer sozialer Medien eher zu Aussagen bereit sind und Inhalte als Rückmeldungen an das betrachtete Unternehmen platzieren als das bisher über andere Formate wie EMail oder Telefon der Fall war. Auf Social-Media-Kanälen veröffentlichte Inhalte würden sich durch ihre Kürze und Prägnanz auszeichnen. Feedback folge oft sehr zeitnah auf von dem Unternehmen veröffentlichte Kommunikationsstimuli. Im Zuge der Diskussion der Vorteile der Methode nennen die Befragten daher auch wiederholt die Eignung der Verfahren für das schnelle Aufdecken neuer, unbekannter Themen oder das Erkennen von Trends. Anhand der Erkenntnisse von Monitoring und Listening im Kontext des ,Trendscouting' sehen sie sich außerdem selbst dazu imstande, Zukunftsszenarien zu prognostizieren:

Befragter: [. . . ] Wo ich sage, ja, ich sage, warum wir das machen, WEIL wir unsere Kunden verstehen wollen, ja. Weil wir SCHNELL Feedback haben wollen. Weil wir AUCH Prognosen machen wollen, Trends erkennen wollen. Und ganz gezielt aus diesen Medien Fragestellungen auch mal, ähm, rausholen wollen [. . . ] (I25/ S17/ Z35-38). 
Eine Woge der Begeisterung für Social-Media-Analysen als Methode löst bei einigen Praktikern ferner das dadurch aus sozialen Medien gewonnene Datenmaterial aus; dieses sei durch Heterogenität bezüglich unterschiedlicher Formate gekennzeichnet. So stellen die Befragten heraus, dass im Rahmen von Monitoring und Listening Daten nicht ausschließlich in Form von geschriebenem Text analysiert werden, sondern auch in visueller und audiovisueller, wie Fotos oder Videos, in die Betrachtungen eingehen. Anhand solcher Daten könnten überaus wertvolle Erkenntnisse gewonnen werden.

Ein weiterer Vorteil der Methode, den praktische Anwender benennen, besteht in ihrer Eignung für die Untersuchung der Einstellungen der Nutzer von sozialen Medien. Mit Monitoring oder Listening könnten in sozialen Medien geäußerte Einstellungen und ihr Wandel im Hinblick auf die von dem deutschen Automobilhersteller ausgesandten Kommunikationsanreize ermittelt werden. Kommunikationswissenschaftliche Studien würden solche Fragestellungen im Rahmen der Persuasionsforschung beleuchten. Diese untersucht zwar klassischerweise die Wirkung von Medieninhalten auf Einstellungen von Rezipienten, doch scheint auch eine Auseinandersetzung mit Inhalten sozialer Medien und deren Wirkung auf die Rezipienten interessant und vielversprechend. Die praktischen Anwender stufen Social-Media-Analysen als geeignet für die Untersuchung von Einstellungen und deren Wandel ein. Der Begriff „Persuasionsforschung“ ist allerdings im untersuchten Datenmaterial nicht enthalten. Dies ist ein weiterer Hinweis darauf, dass die Kenntnisse in sozialwissenschaftlichen Forschungsmethoden und auch Forschungsrichtungen, über die die Befragten verfügen, nicht fundiert oder spezifisch sind. Die praktische Anwendung von Monitoring und Listening wird als eine Ermittlung von „Momentaufnahmen“ und Zeitraumanalysen beschrieben. Ein Wandel in den Einstellungen der Nutzer sozialer Medien könne über mehrere „Momentaufnahmen“ nacheinander oder eine Zeitraumbetrachtung ermittelt werden: ${ }^{16}$

Befragter: [. . . ] Also ich glaube, dass mit Social-Media-Analysen tatsächlich, ähm, (...) zum einen (stockt) SEHR genau festgestellt werden könnte, wie Kunden (...) oder, ja, Kunden oder Marken-Fans oder/ wie die grundsätzlich, ähm, auf/ über die Produkte und die Marke [des Unternehmens des Befragten] oder jedes anderen Herstellers, ähm, aktuell denken. Ähm, wie da so das Standing ist, vor allem so ein gewisser WANDEL. Ich glaube, der lässt sich über eine Social-Media-Analyse recht gut feststellen, wenn man da einen gewissen Zeitraum betrachtet. Weil ich denke, dass (...), egal wie repräsentativ das Ganze ist, aber so ein bisschen, so eine, äh, so diese Schwankung in der Stimmung kann man über so eine Social-Media-Analyse, glaube ich, ganz gut wahrnehmen (I3/ S4/ Z35-43).

\footnotetext{
${ }^{16}$ In Bezug auf die kommunikationswissenschaftliche Persuasionsforschung siehe zum Beispiel: Jäckel, 2011, S. 159 ff.; Schenk, 2009; Wirth, \& Kühne, 2013.
} 
Neben diesen zur ersten Sensibilisierung für die Bandbreite der Vorteile von Social-Media-Analysen genannten Aspekten sind im Untersuchungsmaterial weitere Vorteile der Methode auszumachen. Diese werden gesondert dargestellt. In einem ersten Schritt geht es um die quantitative und, qualitative 'Bipolarität von Social-Media-Analysen und damit um die Möglichkeit Social-Media-Analysen sowohl als quantitatives als auch als ,qualitatives' Verfahren oder als Kombination beider Varianten zugleich nutzen zu können. Daran anschließend wird die Simplizität der Methode behandelt sowie sodann die für die Nutzung von Social-Media-Analysen aufzubringenden geringen Kosten. Zudem geht es um die der Umsetzung von Listening und Monitoring zugesprochene Schnelligkeit und ,Echtzeit' sowie die mit den Verfahren assoziierte Internationalität. Zudem steht die Unmittelbarkeit der Methode als Vorteil im Fokus der Betrachtung.

Sämtliche Vorteile, die anhand des zugrunde liegenden Datenmaterials ausgemacht werden können, sind letztlich auch immer durch die erwähnte mit Social Media einhergehende erhöhte Aussagebereitschaft bedingt. Mitunter auf diese führen die Befragten die Existenz einer großen Datenmenge wie Big Data zurück. Diese liege als Netzöffentlichkeit auf Social-Media-Plattformen herum und könne genutzt werden. Somit sei die Teilnahme der Nutzer sozialer Medien an den Untersuchungen zum einen freiwillig, da die Daten auf deren eigene Initiative hin, ohne Zwang produziert und auf öffentlich zugänglichen Social-Media-Kanälen bereitgestellt würden, zum anderen unwissentlich. Aus dem Unwissen der Nutzer sozialer Medien bezüglich des Rückgriffs auf die Daten im Rahmen von Monitoring und Listening ziehen die Social-Media-Analysten den Schluss, dass die in sozialen Medien vorhandenen oder daraus erhobenen Daten nicht von sozialer Erwünschtheit geprägt sind. Jedoch beschreiben die Praktiker auch diese Annahme in ihren Worten und nutzen den sozialwissenschaftlichen Begriff ,,soziale Erwünschtheit“ nicht; dies demonstriert erneut den Mangel an entsprechenden Kenntnissen. Dieser Einschätzung des aus sozialen Medien erhobenen Datenmaterials entspricht die im Folgenden dargelegte Unmittelbarkeit von Social-Media-Analysen und Kategorisierung von sozialer Medien als ,riesige Grundgesamtheit“, was im Kontext quantitativer Untersuchungen ein Gütekriterium markiert.

\subsubsection{Quantitative und,qualitative' Bipolarität}

In der Möglichkeit, Social-Media-Analysen sowohl als ein quantitatives als auch als ein ,qualitatives' Verfahren zur Anwendung zu bringen, und in gewisser Weise auch in der Kombination beider Varianten erkennen die Praktiker einen wesentlichen Vorteil der Methode. Was diese quantitative und, qualitative 'Bipolarität für die befragten Social-Media-Analysten bedeutet, thematisiert dieser Abschnitt. 
Vorab wurde bereits die große Grundgesamtheit an Daten, die Social-MediaAnalysen als Netzöffentlichkeit zugrunde liegen, angesprochen und mit dem Schlagwort „Big Data“ assoziiert. Monitoring und Listening werden nach Ansicht der praktischen Anwender unter Rückgriff auf diese überaus große Datenbasis durchgeführt, die als „Grundgesamtheit“ von Social-Media-Analysen bezeichnet wird. Im untersuchten Datenmaterial ist die Rede von „Millionen von Aussagen“, welche in sozialen Medien vorhanden sind und aus denen für die einzelnen Analysen Stichproben gezogen werden.

Mit einer via Monitoring oder Listening durchgeführten Untersuchung werde eine große Datenmenge und damit zugleich breite Masse der Nutzer sozialer Medien abgedeckt und untersucht. Social-Media-Analysen basieren damit, auch im Fall von ,qualitativen' Untersuchungen, so führen die Praktiker weiter aus, grundsätzlich auf einer großen Zahlenbasis.

Die hohe Anzahl ermittelter Daten habe den Aufbau einer Datenbank zur Archivierung originaler, aus sozialen Medien erhobenen Daten in einer durchsuchbaren Umgebung erfordert. Im Nachgang von Social-Media-Analysen übertragen einige Social-Media-Analysten sowohl deren Ergebnisse als auch die Daten, auf denen diese beruhen, in eine Datenbank, um jederzeit auf sie zurückgreifen zu können. Von Interesse sei dies zum Beispiel bei Fragestellungen, die an einen bereits in der Vergangenheit untersuchten Analysegegenstand erinnern. Zudem wird mit diesem Vorgehen beziehungsweise dem Anlegen von Datenbanken ein Bezug zu dem Thema „Big Data“ hergestellt, das die Informatik derzeit meist im Kontext von Datenbanken bearbeitet.

Obwohl Social-Media-Analysen, wie die Befragten ferner berichten, auf einer breiten Zahlenbasis beziehungsweise Grundgesamtheit basieren und damit den Anschein einer quantitativen Methode erwecken, generieren die Verfahren nicht selten eine Art von Verständnis, wie dieses sonst nur im Rahmen ,qualitativer “ Untersuchungen erzeugt wird. Dies liege nicht zuletzt daran, dass über Monitoring und Listening Originalinhalte sozialer Medien und damit Originalaussagen in Schriftform von Social- Media-Nutzern und Kunden einen Weg in das betrachtete Unternehmen finden.

Ein weiterer Aspekt, der seinen Beitrag dazu leistet, dass Social-MediaAnalysen von den Befragten ebenso als ,qualitative' Methode beschrieben werden, ist, dass vor allem bei der Variante des Listenings die Datenauswertung unter Einsatz manueller Codierung erfolgt. Dabei handelt es sich um ein Verfahren der Datenauswertung, welches die Sozialforschung vor allem bei qualitativen Inhaltsanalysen oder im Zusammenhang mit der Grounded Theory Methodologie nutzt. 
Somit bleibt festzuhalten, dass die befragten Praktiker Social-Media-Analysen als eine quantitative und, qualitative" Methode zugleich beschreiben und eben genau in dieser Bipolarität einen wesentlichen Vorteil ausmachen, auch im Vergleich mit anderen Forschungsmethoden. Sowohl Monitoring als auch Listening beruhen, so suggeriert das Untersuchungsmaterial, auf einer sehr großen Grundgesamtheit, die aus sämtlichen in sozialen Medien vorhandenen und öffentlich zugänglichen Daten besteht. Social-Media-Analysen schaffen, davon ist weiter auszugehen, Verständnis über oft manuell codierte Originalinhalte sozialer Medien. In ihrer Bipolarität erreicht die Methode, den Eindruck erwecken die Social-Media-Analysten des Automobilherstellers, eine bisher noch nicht gekannte Zusammenführung der Vorteile quantitativer und ,qualitativer Methoden.

\subsubsection{Simplizität}

Social-Media-Analysen zeichnen sich durch ihre Simplizität aus, sind also nach Ansicht der Befragten einfach umzusetzen:

Interviewer: Gut. Wie schätzen Sie persönlich denn dann die Zukunft von SocialMedia-Analysen in der Industrie ein?

Befragter: Wenn man das Ganze, ähm, wie soll ich sagen, entwicklungsbegleitend ein-
setzt, möglicherweise in frühen Phasen zur Entscheidungsfindung, ähm, und (stockt)
den großen Vorteil, dass sie, zumindest nach meinem Verständnis, vergleichsweise auf-
wandsarm immer noch durchführbar sind, dann ist das einfach ein guter zusätzlicher
Kanal. Wenn man es schafft, Social-Media-Analysen in einen Entscheidungsprozess
einzubeziehen, im Sinne von einer/ (...) einer Nutzung der Stärken, also im Sinne
eines, ähm, (stockt) was ich eben vorher angesprochen habe, so dieses, mhm (über-
legend), wenn wirklich tatsächliche Fehler berichtet werden (stockt) oder technische
Probleme oder Dinge, von denen wir einfach sagen, ,,mit unserem Anspruch hinter
einem Produkt sollte so ein Problem gar nicht auftreten“, ist egal, ob es ein echtes
technisches Problem ist oder ob der Kunde nur sagt, ,,das ist aber blöd“, ähm, dann/
(stockt) wenn man mit diesem Fokus ran geht, dann kann man da, glaube ich, auf extrem
(...) aufwandsarme Weise einen sehr guten Rückmelde-Kanal erschaffen, den man,
glaube ich, auch dauerhaft einbinden könnte, insbesondere weil er nicht zu aufwendig
ist (I32/ S17/ Z32-47).

Es wird betont, dass die Methode, sowohl als Monitoring als auch als Listening, durch ihre Einfachheit hinsichtlich der Durchführung von Untersuchungen gekennzeichnet ist. Mit Social-Media-Analysen ließen sich digitale Daten auf einfache Weise analysieren, da die Methode überaus unkompliziert zu handhaben ist. Es sei nur wenig Aufwand für die Umsetzung von Social-Media-Analysen vonnöten. Im Untersuchungsmaterial sind auch keine Unterschiede in Bezug auf die 
Art der Durchführung von Social-Media-Analysen, über Desk Research, mit Tools oder durch einen Dienstleister festzustellen. Es finden sich Begriffe oder Formulierungen wie ,leicht“, ,einfach“, ,,aufwandsarm“, ,nicht schwierig“ oder ,nicht aufwendig“ und „,ohne großen Aufwand“ oder auch „,mit wenig Aufwand“. So scheint der Begriff ,Simplizität“ geeignet, um die entsprechenden Berichte der befragten Social-Media-Analysten als einen weiteren Vorteil der Methode zusammenzufassen. Dem betrachteten Unternehmen der deutschen Automobilindustrie gilt die Simplizität der Umsetzung von Social-Media-Analysen als wesentlicher Vorteil der Methode.

\subsubsection{Geringe Kosten}

Ein ähnlich oft wie die Simplizität von Social-Media-Analysen wiederholter, jedoch ebenfalls nicht tiefgehend diskutierter Faktor ist für die praktischen Anwender der Preis der Methode. Es sind nur geringe Kosten, welche mit den Verfahren des Monitorings und Listenings einhergehen. Social-Media-Analysen gelten in dem hier betrachteten Unternehmen der deutschen Automobilindustrie als eine kostengünstige Methode. Dies insbesondere auch im Vergleich mit anderen Methoden, die zum Beispiel die Abteilung für Marktforschung oder der Unternehmensbereich für Qualität einsetzen. Die geringen mit Social-Media-Analysen verbundenen Kosten haben erheblichen Anteil daran, dass Monitoring und Listening positiv bewertet werden. Im Rahmen dieser Arbeit sind die vergleichsweise geringen Kosten, die Social-Media-Analysen verursachen, daher als ein weiterer zentraler Vorteil festzuhalten, welchen die befragten Social-Media-Analysten des Automobilherstellers mit der Methode verbinden.

\subsubsection{Schnelligkeit und,Echtzeit'}

Erfolgt eine Bewertung von Social-Media-Analysen als Methode, so geschieht dies nur selten ohne den Einbezug des zeitlichen Aspekts. Zeit ist sogar in zweifacher Hinsicht relevant. Zum einen ist die Menge an Zeit, die für die Umsetzung von Monitoring oder Listening aufgebracht und investiert wird, und damit die zeitliche Dauer der Verfahren von Interesse. Dieser Aspekt hängt unmittelbar mit dem als „Simplizität“ benannten Vorteil der Methode zusammen. Zum anderen gehört zu diesem die Geschwindigkeit dazu, mit der die mit Monitoring und Listening gewonnenen Erkenntnisse einen Weg , hinter die Mauern' des betrachteten deutschen Automobilherstellers finden.

So sind die zeitliche Dauer von Social-Media-Analysen und die Geschwindigkeit, mit der sie Erkenntnisse generieren, im Untersuchungsmaterial als weiteres wesentliches Kriterium in der Diskussion um die methodischen Vorteile auszumachen. Dass für die Durchführung von Social-Media-Analysen nur wenig Zeit 
einzuplanen beziehungsweise zu investieren ist, betrachten die Praktiker als erheblichen Pluspunkt. Monitoring und auch Listening seien Untersuchungen von nur geringer Dauer und würden daher auch in enorm hoher Geschwindigkeit Erkenntnisse liefern. Die mit Social-Media-Analysen verbundene hohe Geschwindigkeit vergleichen die praktischen Anwender mit ein paar rasch ausgeführten Klicks an einem mit dem Internet verbundenen Endgerät. Sogar von „Echtzeit“" ist die Rede, was meint, dass über die Verfahren tagesaktuell, gewissermaßen in ,Echtzeit" Inhalte aus sozialen Medien in das Unternehmen geholt werden. Dabei handle es sich zumeist um Reaktionen auf Kommunikationsstimuli:

Befragter: [A] uch hier wieder Echtzeit, wie ist mein Erlebnis mit dem Produkt oder mit einer Dienstleistungs-/ Unternehmens-/ [ . . ]. Also Sie können sofort ECHTZEITAnalysen machen (I22/ S15/ Z33-42).

Somit werden Social-Media-Analysen neben Presseberichten von den Praktikern als der schnellste Kanal angesehen, der Inhalte aus Social Media in Gestalt von Meinungen, Anliegen, Bedürfnissen und Wünschen aber auch Kunden- oder Nutzermotiven sowie deren Dispositionen im Hinblick auf die von dem deutschen Automobilhersteller ausgesandten Kommunikationsstimuli liefert. Monitoring und Listening seien imstande, Antworten auf spezifische Fragen ebenso rasch zu generieren, wie sie beispielsweise eine schnelle Messung der Markenstärke oder von Kampagnenerfolgen sowie ein frühes Erkennen von Problemen mit und Defiziten von Produkten, die auf Social-Media-Plattformen diskutiert werden, leisten könnten. Die Befragten sprechen auch von der Methode als einem „Frühwarnsystem“. Sowohl Monitoring als auch Listening würden zu einem frühen Zeitpunkt Indikatoren aus der in sozialen Medien entstehenden Meinung zu Kommunikationsstimuli des Unternehmens ableiten. Die Rede ist von „Frühwarnindikatoren“, die Social-Media-Analysen liefern. Über Monitoring und Listening würden zum Beispiel in ,Echtzeit‘, quasi unmittelbar nach Markteinführung Rückmeldungen zu einem neuen Produkt gewonnen. Dass die Dauer von Social-Media-Analysen bei ihrer Beurteilung als Methode derart stark gewichtet wird, hängt auch damit zusammen, dass die Befragten Vergleiche mit anderen Methoden, die ihr Unternehmen nutzt, anstellen. Insbesondere wird erneut ein Bezug zu den Verfahren der klassischen Marktforschung hergestellt und betont, dass Monitoring und Listening erheblich schneller durchzuführen sind. ${ }^{17}$ ${ }^{17}$ Auf die mit der Marktforschung und deren Verfahren angestellten Vergleiche wird an
anderer Stelle noch näher eingegangen. 
Es lässt sich also in Bezug auf die Dauer, die eine Durchführung von Social-Media-Analysen beansprucht, und hinsichtlich der Geschwindigkeit, in der Monitoring und Listening schließlich Erkenntnisse aus sozialen Medien in das betrachtete Unternehmen , hineinholen', festhalten, dass durch die Nutzung der Methode Untersuchungen mit wenig Zeiteinsatz beziehungsweise von geringer Dauer, schnell durchgeführt werden können, diese das Unternehmen gewissermaßen in ,Echtzeit" mit Inhalten versorgt. Für die Priorisierung der ermittelten Inhalte ist die Geschwindigkeit, in der diese aus sozialen Medien in das Unternehmen gelangen, von nicht geringer Bedeutung. Durch ein frühes Erkennen von Themen, können diese, so erklären die praktischen Anwender, besser priorisiert werden. Somit werden Social-Media-Analysen von den Befragten als ein Werkzeug eingeordnet, das sehr schnell, ja sogar in ,Echtzeit“, Inhalte generiert. Schnelligkeit und,Echtzeit' gelten als zentrale Vorteile von SocialMedia-Analysen. Oft werden mit der Methode kurze Rückmeldungen zu diversen Kommunikationsstimuli, die zum Beispiel mit der Einführung neuer Produkte einhergehen, in das Unternehmen, hineingeholt". Social-Media-Analysen gelten als Verfahren, das „Frühindikatoren“ für Defizite von Produkten oder Problemen damit liefert. Als „Frühwarnsystem“ gelten Monitoring und Listening auch als Instrumente zur Vorselektion der Marktforschung.

\subsubsection{Internationalität}

Wie zuvor gezeigt, nehmen die Befragten es als Vorteil von Social-MediaAnalysen wahr, dass über diese Rückmeldungen aus sozialen Medien in ,Echtzeit“ gewonnen werden. Mit diesem Aspekt wird im zugrunde liegenden Datenmaterial ein weiterer Vorteil der Methode verknüpft: Die verschiedenen Verfahren sind bei ihrer Lieferung von Rückmeldungen in ,Echtzeit' nicht auf nationalstaatliche Grenzen und damit auf spezifische Verkaufsregionen oder Märkte beschränkt, sondern dazu imstande, weltweit Stimmungsbilder zu erheben. Daher gilt auch die Internationalität als ein Vorteil der Methode:

Befragter: [. . . ] Ich würde es einfach nur (stockt) als Aussage, äh, im/ ich würde jetzt gerne auf den Knopf drücken und (klopft auf den Tisch), und wissen, wie ist die Stimmung weltweit zu, keine Ahnung, wir haben jetzt [das neue Fahrzeug Modell Grün] mit einer größeren Batteriekapazität, und, da würden wir einige Diskussionen haben, da kann ich jetzt nicht warten bis ein Long-Lead-Medium in drei Wochen rauskommt mit einem Riesen-Bericht und das bewertet, aber ich könnte sehen, was spricht Community eins und Community zwei, wie ist da/ wie haben sie es gelauncht, (stockt) wie gehen da die Diskussionen, was sind die ersten Erfahrungen der Kunden und so weiter, in dem Sinne (I22/ S10-11/ Z48-7). 
Social-Media-Analysen ermöglichen laut ihren praktischen Anwendern Untersuchungen von weltweitem Ausmaß. Monitoring und Listening würden überall funktionieren und könnten international eingesetzt werden. Mehrere Märkte könnten zugleich, im Rahmen von nur einer Untersuchung betrachtet und miteinander verglichen werden. Anhand des Vergleichs unterschiedlicher Verkaufsregionen seien die Besonderheiten der Märkte zu erkennen. Diskutiert wird dieser Vorteil der Verfahren unter dem Aspekt der hohen Reichweite von Social-MediaAnalysen:

Befragter: Genau. ODER auch, äh, was auch, äh, klar ist, äh, Reichweite und so. Also du kannst natürlich hier von Honolulu, Hawaii über USA bis/, äh, ohne hinfliegen zu müssen. Oder ohne (...), äh, dort Leute rekrutieren zu müssen oder Ähnliches. Die machen das ja alle freiwillig und wissen gar nichts davon. (...) Dort (I15/ S5/Z39-42).“

Die Befragten erkennen in der Methode also den Vorteil, internationale Erkenntnisse basierend auf einer hohen Reichweite generieren zu können und dies ohne dabei selbst den eigenen Standort verlassen zu müssen. Dies wird als praktisch und angenehmen bewertet. Damit ist der als Internationalität der Methode benannte Vorteil wiederum mit dem Vorteil der Simplizität verbunden.

\subsubsection{Unmittelbarkeit}

Über Social-Media-Analysen werden, so berichten die Praktiker, Informationen, etwa in Gestalt von Meinungen und Rückmeldungen erhoben; dieses Feedback zeichne sich durch eine besondere Eigenschaft aus:

Befragter: [... ] [U]nd es gibt ja nichts Schöneres als eine ungefilterte MEINUNG und keine beeinflusste Meinung, die man dann entsprechend auswertet und dann (atmet ein) reflektiert wieder in das Produkt in Form von Anforderungen einfließen lässt (I20/ S1/ Z39-41).

Dem Feedback, das über Monitoring und Listening gewonnen wird, wird ein eigene Qualität zugeschrieben. Es ist die Rede von „echten“, ,authentischen“, „ungefilterten“, „unbeeinflussten“ Inhalten, die nicht in einer künstlich erzeugten Umgebung, wie einem Labor, generiert werden, sondern im „,realen Leben“ oder der „Wirklichkeit“ der Nutzer sozialer Medien, die diese Inhalte erzeugen. Diese von den Befragten als Vorteil der Methode dargestellten Eigenschaften der Inhalte, die Social-Media-Analysen erheben, betrachtet diese Arbeit als Unmittelbarkeit.

Die Unmittelbarkeit der Inhalte sozialer Medien assoziieren die Praktiker mit einem Aspekt des Sozialen, der Social Media auszeichnet. Aufgrund dieses sozialen Aspekts, den sie sozialen Medien zuschreiben, betrachten sie die 
über Social-Media-Analysen erhobenen Daten als „,authentisch“ und ,real“. Die mithilfe der Methode gewonnenen Erkenntnisse seien ,,ungefiltert“" oder auch ,unbeeinflusst". Andere umschreiben dies nicht mit ausdrucksstarken Adjektiven, die suggerieren, dass alle auf eine andere Art ermittelten Daten minderwertig sind, sondern sprechen nur von ,nicht gegebenen Reaktivitätsproblemen“. Damit meinen sie, dass Inhalte sozialer Medien ohne Einfluss sozialer Erwünschtheit entstanden sind.

Monitoring und Listening liefern also ,unmittelbare“, aber dadurch auch ,ungestützte" Rückmeldungen und Informationen in direkter Form, deren besonderes Kennzeichen ihre Authentizität ist. Diese Unmittelbarkeit gilt als weiterer Vorteil, der Social-Media-Analysen als Methode. Es ist die Rede von ,direkter Rückkoppelung“ zu den Social-Media-Nutzern und damit gegebenenfalls auch zu Kunden des Automobilherstellers. Ebenso zeigt sich eine Rückkoppelung einiger Vorteile von Social-Media-Analysen untereinander:

Es fällt einem bei einer Social-Media-Analyse nur wesentlich einfacher, weil der Draht, den man zu den/ zur Zielgruppe hat, ein sehr viel direkterer ist. Das heißt, wenn was gut funktioniert, dann bekommt man das auch direkt zurück, ja. Dieses direkte Feedback, ähm, auch wahrzunehmen ermöglicht es einem (...) am offenen Herzen quasi noch Veränderungen vorzunehmen. [. . . ] Ja, ich meine, gerade die Chancen, die man hat, ich f/ die Chancen, die man hat, auch Informationen zu verdichten, Menschen geben sehr viel preis über die Kanäle. Man hat die Möglichkeit, jetzt in Deutschland weniger, aber in anderen Ländern, die auch mit dem Datenschutz ein bisschen offener umgehen, ähm, Möglichkeiten, Dinge zu verdichten, ja. Big Data Analysen zu machen, ähm, die einem (...) sehr viel Insights auch geben, ja. Wir nutzen das, um, es nennt sich Custom Audiences, das heißt, wenn wir in einer Vermarktung, ähm, Werbung ausspielen, dann hilft uns eine Analyse daraus, ähm, festzustellen, wenn jemand Interesse hat an einem [Fahrzeug Modell Grün], dann hat er auch Interesse zu einem gesteigerten Maß an Urban Gardening. Oder, ähm, ich weiß nicht, äh, Lifestyle orientierten Themen, ähm, oder an Mode, ja (I6/ S5/ Z22-48).

Der als „Unmittelbarkeit“ bezeichnete Vorteil von Social-Media-Analysen hängt wieder direkt mit weiteren Vorteilen der Methode, wie deren Simplizität, deren Schnelligkeit und ,Echtzeit‘ oder auch deren Internationalität, zusammen. Zwischen diesen Vorteilen der Methode besteht laut den Befragten eine wechselseitige Ergänzung. 


\subsubsection{Nachteile der Methode}

Zentrale methodische Vorteile, die gemäß der Wahrnehmung der befragten Praktiker den Social-Media-Analysen inhärent sind, wurden betrachtet. Deshalb sind nun ebenso die Nachteile der Methode, die ihre praktischen Anwender sehen, zu umreißen. Es wurden bereits einige spezifische Charakteristika sozialer Medien herausgearbeitet. Diese beeinflussen wiederum deren Untersuchung oder vielmehr die Betrachtung ihrer Inhalte, etwa durch Social-Media-Analysen. Aus den besonderen Eigenschaften sozialer Medien werden unmittelbare Nachteile von Monitoring und Listening abgeleitet. Solche Nachteile, die zwar angesprochen, aber nicht weiter erläutert werden, die aber trotzdem in dieser Forschungsarbeit relevant sind, werden nun gezeigt ehe anschließend weitere wesentliche Nachteile von Monitoring und Listening in den Fokus gerückt werden.

Die praktischen Anwender heben hervor, dass sowohl Monitoring als auch Listening niemals Erkenntnisse im Hinblick auf eine sogenannte „große Mitte“ liefern, da ihre Ergebnisse auf Extrema beruhen. Diese Einschätzung der Methode rekurriert darauf, dass zum gegenwärtigen Zeitpunkt davon ausgegangen werden muss, dass die Nutzer sozialer Medien weder eine gesamte Gesellschaft repräsentativ abbilden noch die für die Automobilindustrie und deren Fragestellungen relevanten Gruppen, die sich mitunter anhand soziodemographischer Merkmale voneinander unterscheiden. Diesen Aspekt sozialer Medien werten die Befragten als einen Nachteil der Methode. Social-Media-Analysen seien lediglich dazu imstande, auf extremen Positionen beruhende Ergebnisse abzubilden, würden keine Erkenntnisse im Hinblick auf die Gesamtgesellschaft oder deutsche Automobilindustrie liefern und seien nicht repräsentativ in Bezug auf die für ihre jeweilige Fragestellungen relevanten sozialen Gruppen.

Ferner ist festgehalten, dass die Ergebnisse von Social-Media-Analysen ,unstrukturiert" sind. Zudem wird ihre Authentizität in Frage gestellt. Die Ergebnisse von Monitoring und Listening seien mit Vorsicht zu genießen. Wegen ihrer mangelnden Struktur sei die Authentizität nicht nachprüfbar. Damit einher gehe die „,undefinierte Aussagekraft“, die den Ergebnissen von Social-Media-Analysen anhaftet. Alle diese Aspekte würden den Umgang mit den Ergebnissen erschweren. Dies sei ein Nachteil der Methode.

Bei den mit den Verfahren einhergehenden Quantitäten und der großen Datenmenge im Sinne von Big Data, auf welcher die Untersuchungen beruhen, handle es sich um eine vermeintlich hohe Zahl. Ferner wird der der Zahlenmacht entgegengesetzte, qualitative ' Anteil von Social-Media-Analysen, die, qualitative Datenauswertung ' in Form einer manuellen Codierung, als minderwertig im Vergleich zu quantitativen oder statistischen Datenanalysen bewertet. Weder quantitative noch 
, qualitative' Social-Media-Analysen scheinen gut zu funktionieren und eine Berechtigung bei dem betrachteten deutschen Automobilhersteller zu haben. Manuell durchgeführte Social-Media-Analysen bewerten die einen als zu teuer, die anderen wegen ihrer, qualitativen' Natur per se als schlecht. Automatisierte und rein quantitativ umgesetzte Untersuchungen werden auch nicht nur positiv eingeschätzt. Die Methode wird den Ansprüchen schlichtweg nicht gerecht:

Befragter: [. . . ] Wie kann man es am besten angehen? Was wir ja gesehen haben, es gibt weder die e/ Lösung, wo es nur Menschen machen, weil es zu teuer wird, es gibt aber auch keine Software, die das relativ gut automatisiert (räuspert sich) irgendwie abdecken kann. Ähm, gerade, wenn man jetzt nicht nur auf Englisch das mal als Pilot macht oder auf Deutsch, sondern irgendwie am Ende des Tages auch mit 17 Sprachen hantieren muss und ein jeder auch weiß, dass irgendwie nicht jeder im Internet irgendwie Oxford-Englisch spricht, na, was wir in den letzten Jahren auch immer gesehen haben, gibt viele Dienstleister, relativ viele Firmen, die uns da was verkaufen wollten, und allein der Punkt, dass relativ viele Leute schreiben „I love my fucking [Marke des Unternehmens des Befragten]" ist halt so ein Punkt, wenn ich jetzt nur Wort für Wort mir anschaue, ist fucking rot, äh, love ist grün, das heißt der Tweet oder die Nachricht wird halt sowohl positiv als auch negativ zugeordnet, das heißt diese, äh, Analyse des GANZEN SATZES ist schon mal, im Prinzip essentiell für uns, was am Ende des Tages auch wieder immer weniger/ immer weniger, ähm, Softwaren KÖNNEN und die, die es können, kosten auch wieder mehr. Na, also, äh, ist ein relativ (...) schwieriges Thema und, ich habe, ehrlich gesagt, noch KEINE Lösung und keinen Anbieter gefunden, der jetzt, zumindest mal UNSEREN ANSPRÜCHEN gerecht wird. Allein aus Marketingsicht, global, das alles abzudecken (I9/S3/Z10-26).

Ein weiterer Nachteil von Monitoring und Listening, von dem die Praktiker glauben, dass er die Nutzung der Verfahren aktuell deutlich erschwere, besteht in den aktuellen datenschutzrechtlichen Bestimmungen vieler Länder. So seien Social-Media-Analysen momentan durch Datenschutzbestimmungen erheblich eingeschränkt, so dass noch nicht auf alle in sozialen Medien vorhandene Daten zugegriffen werden kann. Dies habe Auswirkungen auf die Aussagekraft der Ergebnisse, die die Methode generiert.

Bezüglich der durch Social-Media-Analysen gewonnen Ergebnisse ist dem untersuchten Datenmaterial zu entnehmen, dass diese ebenso wie die mithilfe von anderen Methoden hervorgebrachten als Vergangenheitsbetrachtungen einzuordnen sind. Eine Vorhersage der Zukunft könnten sie nicht leisten:

Befragter: Also für mich sagen So/ können Social-Media-Analysen nicht zeigen, wie man es in Zukunft macht. Man kann/ das ist für mich eine Vergangenheitsbetrachtung. Man versucht, da ein bisschen die Zukunft abzuleiten, aber, ähm, das geht wie beim Aktienmarkt, äh, kann ich schön die Charge und die Verläufe und irgendwas eintragen, (atmet ein) hat dann mit der Realität selten was zu tun (I17/ S7/ Z22-26). 
Dennoch scheint in dem Unternehmen die Gefahr eines nicht reflektierten Umgangs mit den durch die Verfahren des Monitorings und Listenings gewonnenen Ergebnissen zu bestehen. Manche ihrer praktischen Anwender würden dazu neigen, die Ergebnisse falsch zu interpretieren. Diese würden mitunter , für bare Münze genommen` ohne über ihre Entstehung unter Anwendung der Methode zu reflektieren. Das Risiko des nicht reflektierten Umgangs mit den Ergebnissen wird als Nachteil der Verfahren oder sogar als Gefahr bewertet. Social-Media-Analysen würden keine repräsentativen Ergebnisse liefern, so dass sie nicht geeignet seien als Grundlage für strategische Entscheidungen zu dienen.

Ein weiteres Defizit der Methode erkennen Befragte darin, dass Ergebnisse von Social-Media-Analysen nicht graphisch darstellbar sind. Es sei erforderlich, diese, ebenso wie das Datenmaterial, auf dem sie basieren, durchzulesen, um sie verstehen zu können. Eine graphische Darstellbarkeit der Ergebnisse sei für den Automobilhersteller jedoch ausschlaggebend, um diese anderen Abteilungen des Unternehmens oder auch höheren Hierarchieebenen verständlich machen zu können. Aussagekräftige Graphiken, welche keiner weiteren Erklärung bedürfen, seien unabdingbar.

Ferner bewerten die Social-Media-Analysten das Thema „CRM“ als einen Nachteil der Methode. Durch die Verfahren würden zwar Botschaften von Nutzern sozialer Medien, die diese über Social-Media-Kanäle an das Unternehmen senden, ermittelt, doch sei weder über Monitoring noch Listening beziehungsweise durch den Verfahren inhärente Mechanismen ihre Weiterleitung an verantwortliche Unternehmenseinheiten möglich. Aus Gründen des Datenschutzes bleibe die Identität der Absender von Botschaften auf Social-Media-Plattformen unbekannt, so dass eine Beantwortung unmöglich erscheint.

Monitoring und Listening konfrontieren die Praktiker des Weiteren mit dem Vorwurf methodischer Schwächen im Allgemeinen. Damit einher geht Kritik am Umgang mit den Verfahren, den der betrachtete Automobilhersteller und seine Agenturen pflegen: 
Befragter: [...] $\mathrm{Äh}$, also ich bin als Marktforscher halt damit konfrontiert worden, dass es, äh, dieses Phänomen hochgekommen ist, dass sehr viele Start-ups, ähm, die sich, ähm, mit Social-Media-Analyse beschäftigen, äh, relativ aggressiv im Unternehmen aufgeschlagen sind, um, äh, zu requirieren und das auch immer wieder auf fruchtbaren Boden gefallen ist. Ich dann aber auch oft den Eindruck hatte, das sind Leute, die es zwar, äh, IT-technisch können, die aus der IT kommen, äh, aber die, äh, die methodologische Seite mehr oder weniger komplett außen vorlassen, äh, ähm, und, sei es bewusst, sei es unbewusst, weil sie wirklich die entsprechenden Skills nicht haben, teilweise das Blaue runter vom (stockt) Himmel runter versprechen, was das alles kann, was das alles ersetzt, äh, wie gut das alles funktioniert und so weiter mehr. (Atmet ein) das berührt eine Menge Fragen, äh, zum Beispiel, was man damit überhaupt alles SINNVOLL bearbeiten kann. Ähm, auch das Thema, äh, einer automatisierten Auswertung, äh, da werden die Probleme in der Regel von den Anbietern, die das, äh, können oder vorgeben zu können, nicht genügend, äh, betrachtet, ähm, dass einfach schon das Einordnen der Sentiments, äh, sehr, sehr problematisch ist, geschweige denn, dass man da jetzt irgendwelche tieferen, (atmet ein) äh, Erkenntnisse dazu bekommt (I18/ S1/ Z33-47).

Den Dienstleistern wird ein Mangel an den für Social-Media-Analysen erforderlichen methodischen Kompetenzen vorgeworfen; ihr Umgang mit der Methode wird vielmehr noch als unreflektiert konstatiert. Die Agenturen würden zwar über IT-Kenntnisse verfügen, doch es fehle an sozialwissenschaftlichen Kompetenzen. Auch innerhalb des betrachteten Unternehmens seien auf der Seite derjenigen Praktiker, die mit Agenturen kooperieren, nur sehr geringe Kenntnisse sozialwissenschaftlicher Forschungsmethoden vorhanden, die zu einem reflektierten Umgang mit den Verfahren unter Einbezug deren methodischer Schwächen befähigen würden. In Bezug auf die Zusammenarbeit mit Dienstleistern werden weitere Kritikpunkte, die zugleich als Nachteile der Methode gewertet werden, geäußert: Zum einen erkennen Social-Media-Analysten eine Gefahr darin, sich in ein Abhängigkeitsverhältnis von einer Agentur zu begeben; zum anderen nehmen praktische Anwender eine Unbeständigkeit der Dienstleister wahr, welche sich beispielsweise darin äußert, dass sie sich immer wieder umbenennen.

Diese angeführten Nachteile von Social-Media-Analysen haben einen ersten Einblick in die Bandbreite gegeben, die die Befragten in Bezug auf die Methode erkennen lassen. Im Folgenden stehen die Nachteile im Fokus, die anhand der Berichte der Praktiker als ihre bedeutendsten auszumachen sind. In einem ersten Schritt werden einige Nachteile zusammengefasst, die in einem Widerspruch zu manchen schon als Vorteile dargelegten Aspekten stehen. Sodann werden wesentliche technische Defizite der Verfahren, die die Befragten anführen, behandelt. Anschließend geht es um den Aspekt der mangelnden Repräsentativität, 
der anhand des Untersuchungsmaterials als zentraler Nachteil von Social-MediaAnalysen auszumachen ist. Daraufhin wird der von den Praktikern als Unwissen in Bezug auf die Sender einzelner Social-Media-Botschaften beschriebene Nachteil von Monitoring und Listening beleuchtet. Im Folgenden ist die mit SocialMedia-Analysen einhergehende, als Nachteil bewertete Unklarheit hinsichtlich der Relevanz von Themen Gegenstand der Betrachtung. Zum Abschluss geht es um die von einigen praktischen Anwendern vermisste Erkenntnistiefe der Methode.

\subsubsection{Nivellierung der Vorteile}

Dieser Abschnitt über die Nivellierung der Vorteile von Social-Media-Analysen stellt insgesamt vier Aspekte dar. Die Befragten haben diese sowohl als Nachteile von Monitoring und Listening berichtet als auch genauso oder zumindest sehr ähnlich als Vorteile der Methode thematisiert.

Es handelt sich zum Ersten um den für die Umsetzung von Social-MediaAnalysen erforderlichen Aufwand, der im Rahmen der Darstellung der Vorteile der Methode als Simplizität behandelt wurde. Neben den Befragten, die auf die Simplizität von Social-Media-Analysen als Vorteil der Methode eingehen, finden sich ebenso Stimmen, die Monitoring und Listening als aufwändige Methode beschreiben:

Befragter: [ . . ] Ähm (...), grundsätzlich, ähm (...), ist man halt immer vorsichtig bei der (stockt) Interpretation, weil diese Auswertungen ja auch relativ schw/ aufwendig, schwierig wahrscheinlich nicht, aber aufwendig sind. Also, wann wird was posit/ oder wann ist was positiv konnotiert und wann negativ? Und ist das eigentlich durch das Raster gefallen, weil der halt aus Kostengründen, das einfach nur durchgehauen hat und die Begriffe hat zählen lassen (I2/ S2/ Z30-35)?

Zwar sind diese Stimmen wesentlich seltener zu vernehmen als die entgegengesetzten, doch sie existieren. Social-Media-Analysen werden insofern als mit einem Aufwand verbunden dargestellt, als es nicht einfach sei, mit aus sozialen Medien erhobenen Daten ein ,einheitliches“ und auch „stimmiges“ Bild zu generieren. Um dies zu erreichen, sei viel Zeit aufzuwenden. Monitoring und Listening werden als Arbeit und methodische Herausforderung angesehen.

Zweitens wird die Relation zwischen den Kosten für Monitoring und Listening und dem entstehenden Nutzen als Nachteil betrachtet. Dieser als ein Nachteil angeführte Aspekt hängt eng mit dem Aspekt des Aufwands zusammen. Monitoring und Listening werden, anders als in der Diskussion um die methodischen Vorteile von Social-Media-Analysen, ebenso als nicht kostengünstige Verfahren beschrieben. Die Social-Media-Analysten weisen darauf hin, dass das Verhältnis 
zwischen den Kosten, die die Durchführung der Verfahren verursacht, und dem Nutzen, den ihre Ergebnisse versprechen, nicht stimmt. Vor allem ein Monitoring sozialer Medien über 24 Stunden hinweg und die Auswertung der Daten über manuelle Codierverfahren sei teuer und würde daher auch weitestgehend vermieden. Manche Befragte raten aufgrund der Relation von Kosten und Nutzen sogar ganz von einer Durchführung von Social-Media-Analysen ab.

Der dritte Aspekt besteht in der Verbindung mit Social-Media-Analysen diskutierten ,Echtzeit‘. Der Methode wird die Fähigkeit abgesprochen, Ergebnisse zeitgenau, also in ,Echtzeit' liefern zu können:

Befragter: [... ] Auch, wenn die jetzt relativ lange sammeln mussten, das ist halt kein Volumenmodell, ähm, wo die, äh, äh, Zahl die Leute, die sich dazu äußern, gigantisch groß ist. Also, ich glaube, die haben drei oder vier Monate dann auch Statements gesammelt, nach dem Launch. Also, so die Fantasie, heute launchen wir, morgen haben wir Feedback, ist auch dann, äh, wohl übertrieben (I18/ S4/ Z37-41).

Viertens und letztens richtet sich das Augenmerk auf die besonderen Gegebenheiten, die bei Social-Media-Analysen für den chinesischen Markt oder Sprachraum zu beachten sind; damit wird eine Gegenposition zu der als Vorteil dargestellten Internationalität behandelt. Das Thema „China“ und die Eignung von SocialMedia-Analysen für die Betrachtung des chinesischen Sprachraums oder Markts sei ein Nachteil der Methode. Mit der Nennung dieses Nachteils wird der Vorteil der Internationalität von Monitoring und Listening zum Teil nivelliert. Praktiker berichten China betreffend von sprachlichen Einschränkungen und informationspolitischen Restriktionen oder sind der Ansicht, dass Social-Media-Analysen für China aus den genannten Gründen überhaupt nicht möglich oder umsetzbar sind. Es gebe Zugangsschwierigkeiten zu den Daten; diese bestünden auch darin, dass in China andere soziale Medien genutzt werden als außerhalb.

Somit führen die Social-Media-Analysten insgesamt vier Nachteile der Methode an, die zugleich als Nivellierung von schon als solchen dargestellten Vorteilen des Monitorings und Listenings aufgefasst werden können.

\subsubsection{Technische Defizite}

Im Zuge ihrer Schilderungen der Nachteile von Social-Media-Analysen kommen die praktischen Anwender auch auf noch immer gegebene technische Defizite der Methode zu sprechen. Vorranging beziehen sie sich auf automatisiert umgesetzte Social-Media-Analysen und stellen das Thema „Spracherkennung“, ein automatisiertes Erkennen von Tonalität und Ironie, in den Fokus ihrer Ausführungen. 
Die Befragten nehmen deutliche Mängel hinsichtlich automatisierter Tonalitätsanalysen wahr. Diese sind jedoch zumeist fester Bestandteil der Untersuchungen. Weder bei Agenturen noch bei Tools sei die Fähigkeit der automatisierten Spracherkennung so ausgereift, dass Tonalitäten richtig eingeordnet werden können. Umgangssprachliche Äußerungen, die in sozialen Medien nicht die Ausnahme, sondern die Regel darstellen, könnten nicht erkannt und daher in Bezug auf ihre Bedeutung auch nicht eindeutig ausgemacht werden. Zwar könnten Tonalitätsanalysen reine Schriftsprache erkennen, doch umgangssprachlich formulierte Inhalte seien noch nicht zu bewältigen:

Befragter: Ähm, gerade das Thema Natural Language Processing. Das Qualitative ist für mich, auch heutzutage, noch eine massive Herausforderung. Ähm, dass man eben nicht sagen kann, wie positiv, wie negativ, ähm, ist irgendwas rübergekommen oder schreiben Menschen darüber. Auch, ähm, inhaltlich zu verdichten [. . . ]. Und die meisten Tools, ähm, würden ein „Scheiße nochmal, ist das Ding geil“ eher negativ einordnen, ja. Einfach nur von der Art der maschinellen Verarbeitung. Ja, und, ähm, dass auch mit Emoticons oder Emojis oder so was sehr schwer Tool gestützt gearbeitet werden kann. Das heißt, qualitative Analysen sind, glaube ich, immer noch sehr, sehr schwer da auch skalierbar umzusetzen und nicht mit einer Heerschar an, ähm, Analysten, die eine Kampagne bewerten, zu bewerkstelligen ist (I6/ S11/ Z24-36).

Weder Dienstleister noch Tools hätten so ausgereifte Fähigkeiten in Computerlinguistik oder auch Natural Language Processing, dass die Ergebnisse von Tonalitätsanalysen verlässlich seien. Die Befragten heben hervor, dass die Informatik in Bezug auf maschinelles Lernen noch deutliche Hürden zu überwinden und Herausforderungen zu meistern hätte. Beispielsweise seien automatisierte Social-Media-Analysen nicht dazu in der Lage, Emojis oder Emoticons zu erkennen. Dabei handle es sich jedoch um in sozialen Medien durchaus übliche Zeichen der Kommunikation. Somit betonen die Praktiker sowohl Defizite in Bezug auf die automatisierte Erkennung von Tonalität als auch hinsichtlich anderer Kommunikationszeichen als Sprache und fassen beide als mit technischer Spracherkennung verbundene Nachteile zusammen. Inhalte sozialer Medien könnten noch nicht automatisiert ausgewertet werden. Da Tonalitätsanalysen meist als ,qualitative" Untersuchungen im Rahmen von Monitoring und Listening angesehen und auch entsprechend bezeichnet werden, ist oft auch die Rede davon, dass diese ,, noch nicht“ oder ,nur unzureichend“ funktionieren, sofern die Durchführung automatisiert erfolgt. Automatisiert umgesetzte Untersuchungen würden in der Regel einer manuellen Nachbearbeitung bedürfen, damit ihre Ergebnisse überhaupt verwendet werden können. In der negativen Bewertung von „qualitativ“ genannten, allerdings rein automatisiert durchgeführten Tonalitätsanalysen ist der Grund dafür zu sehen, dass Social-Media-Analysen in ihrer Aussagekraft als ungeeignet für strategische Ableitungen gelten. 
Bezogen auf eine automatisierte Spracherkennung im Rahmen von Monitoring sowie Listening wurde das Thema „Ironie“ als zweiter wesentlicher Aspekt genannt, den die Praktiker an Social-Media-Analysen kritisieren. Ähnlich wie bei Tonalitäten könnten die Verfahren, sofern diese automatisiert durchgeführt werden, Ironie nicht erkennen:

Befragter: [... ] Und das geht ja ganz gut für einfache Aussagen, ,ja, wir finden [Marke des Unternehmens des Befragten] super" heißt dann mit großer Wahrscheinlichkeit, ähm, positives Sentiment. Aber auch nur mit großer Wahrscheinlichkeit, kann auch durchaus ironisch gemeint sein, ,ja, ich war neulich beim Service und musste da hinfahren; [Marke des Unternehmens des Befragten] ist echt super". Da haben wir wieder so die einfache Lösung, ,ja, [Marke des Unternehmens des Befragten] ist echt super“", ganz toll, aber, wenn ich dann natürlich den Satz vorher anschaue und die beiden korreliere, was ja eine bisschen fortschrittliche Sentiment-Analyse dann bedingt, würde man sagen, ,ah, das wohl eher ironisch gemeint“, man hätte halt dann ein negatives Sentiment dahinter, ja. Also da ist so der, glaube ich, der Spannungsbereich, wo man auch dann auch an die Grenzen stößt von dem Ganzen (I25/ S14/ Z38-46).

Auch im Hinblick auf das Thema „Ironie“ sei es derzeit notwendig, Ergebnisse, die aus automatisiert umgesetztem Monitoring oder Listening hervorgegangen sind, manuell zu überprüfen. Weder Tools noch Dienstleister, die automatisierte Social-Media-Analysen anbieten, würden den Kontext einzelner Aussagen in ihre Bewertung einbeziehen. Da sich Ironie oft erst durch den Kontext von Aussagen erschließe, seien Fehlcodierungen nicht selten.

Wie in Bezug auf die Spracherkennung bei automatisiert durchgeführten Social-Media-Analysen schon festgestellt, sind die Technologie beziehungsweise die Algorithmen, die diesen aktuell zugrunde liegen, nach Ansicht der Befragten noch nicht ausgereift. Dies hängt damit zusammen, dass sie noch nicht ausreichend trainiert wurden, um Inhalte intelligent zu erkennen und hinsichtlich ihrer Tonalität oder Ironie korrekt einzuordnen. Jedoch kann die Mehrheit der Befragten die hinter einer automatisiert durchgeführten Social-Media-Analyse stehende Technologie weder erklären noch deren Defizite genau beschreiben. Stattdessen ist von einer „Technik, die noch nicht so weit ist“, oder von „Fragen der Technik, die noch nicht geklärt sind" die Rede. Nur die quantitativen Ergebnisse der automatisierten Verfahren gelten als brauchbar, doch diese generieren nicht die von der Methode erwarteten Erkenntnisse: 
Befragter: [. . . ] Also die Aufbereitung/ (stockt), also momentan ist es hochgradig automatisiert worden (...), ah, und das ist für mich jetzt wieder eher so ein bisschen (stockt) ein Schritt zurück, weil es zu sehr rein wieder auf die Zahlen runter gebrochen wird. [.. . ] [M]ich interessiert nicht, ob ich jetzt 35 Rückmeldungen oder 380 Rückmeldungen habe, sondern ich will halt mal grob wissen, was für Rückmeldungen habe beziehungsweise, wenn ich ein konkretes Problem habe, dass ich, ah, nachschauen kann, wie ist denn da draußen momentan die Stimmung (I21/S9/ Z23-30)?

\subsubsection{Repräsentativitätsmangel}

Repräsentativitätsmangel ist ein weiterer Hauptkritikpunkt an Social-MediaAnalysen:

Befragter: //Was es weiter nicht leisten kann, ist eben (stockt) eine und damit im Zusammenhang (stockt) eine quantifizierte Abschätzung, die eine repräsentative Basis hat, und ich muss immer sehen, dass ich (...) eigentlich keinen Diskurs mehr führen darf (I32/ S17/ Z18-20).

Wie die zuvor dargestellten technischen Defizite wird der Mangel an Repräsentativität von fast allen Social-Media-Analysten angeführt, wenn sie über Grenzen der Methode sprechen. Dies hängt auch damit zusammen, dass der Automobilhersteller vor allem auf Informationen quantitativer Art setzt und von einer ,Macht der Zahlen“ beherrscht wird. Das betrachtete Unternehmen offenbart anhand der Ausführungen der Praktiker eine ,quantitative Denkweise oder ,quantitative Logik‘. Da es als Organisation in Zahlen ,denkt' und , handelt', ,zählen' nur quantitative Inhalte. Der Wert von Erkenntnissen, auch derjenigen, die über Social-Media-Analysen gewonnen werden, bemisst sich, so ist festzustellen, anhand von Quantitäten. Nur Ergebnisse, die quantitativ und repräsentativ sind, gelten als ,vernünftig“. Doch Repräsentativität sehen die Befragten bei Monitoring und Listening nicht gegeben und erkennen dies als einen zentralen Nachteil von Social-Media-Analysen. Es handelt sich dabei um einen Nachteil der Methode, der auch im Umgang mit deren Ergebnissen beständig mitschwinge und nicht auszublenden sei. Zutage treten könne dieser Nachteil mitunter in einer immerwährenden Unklarheit in Bezug auf das Vorhandensein von Repräsentativität. Es entsteht gar der Eindruck, dass alle Social-Media-Analysen mitsamt ihren Ergebnissen von der Frage nach ihrer Repräsentativität überlagert werden. Weder mit Monitoring noch Listening seien ,belastbare“ Informationen zu ermitteln, mit denen im Anschluss weitergearbeitet, auf deren Basis Strategien entwickelt und Entscheidungen getroffen werden können. Dies liegt, so erläutern die SocialMedia-Analysten, an der mangelnden Repräsentativität der Ergebnisse, die die Methode liefert, und an ihrer fehlenden ,statistischen Relevanz“: 
Befragter: (...) Also ich glaube, (...) eine quantitativ belastbare Aussage zu geben, dass genau das und das dabei rauskommt, um auf Zahlen/ (stockt) anhand von Zahlen, Daten, Fakten zu entscheiden, (stockt) ist, glaube ich, schwierig (I28/ S9/ Z19-24).

Eng mit dem kritisierten Mangel an Repräsentativität hängen die Schwierigkeiten der Einordnung von über Social-Media-Analysen gewonnenen Erkenntnissen in Bezug auf ihre Relevanz und Bedeutung zusammen. Dieser Aspekt wird im weiteren Verlauf dieser Arbeit noch näher betrachtet, aber aufgrund seines Zusammenhangs mit dem Repräsentativitätsmangel bereits an dieser Stelle erwähnt; er gilt als Hauptursache für die Einordnungsschwierigkeit der Themenrelevanz. Es gibt zwar Überlegungen, die Repräsentativität „künstlich“ durch Vergleiche zu erzeugen, doch dieser Ansatz scheint keine Lösung, die dem Repräsentativitätsmangel entgegengesetzt werden kann.

So halten die Befragten fest, dass die Methode keine Erkenntnisse zu einer relevanten Grundgesamtheit, wie der gesamten Kundschaft des Automobilherstellers, generieren kann; über Social-Media-Analysen seien keine Rückschlüsse darauf möglich:

Befragter: $[\ldots][\mathrm{H}]$ aben ihnen in den meisten Fällen abgeraten, weil wir gesagt haben, ihr braucht was oder ihr wollt eine Aussage für (...), für eine Grundgesamtheit der Kundschaft und das werdet ihr bei der Social Media jetzt nicht bekommen, sondern da kriegt ihr irgendwelche Tendenzen oder vielleicht neue Ideen oder neue Hypothesen, aber das wird es tatsächlich da nicht geben [. . .] (I2/ S2/ Z5-8).

Statt für relevante Grundgesamtheiten repräsentative Ergebnisse zu liefern, seien Social-Media-Analysen als Methode dazu geeignet, Tendenzen zu ermitteln oder Einblicke in die in sozialen Medien vorherrschende Gefühlslage zu gewähren. Im Hinblick auf das Thema „Repräsentativität“ gilt dies als Nachteil der Methode oder auch als Kehrseite der Verfahren. Mangels Repräsentativität könnten aus den Erkenntnissen von Social-Media-Analysen keine Rückschlüsse auf relevante Grundgesamtheiten, die in sozialen Medien auch gar nicht anzutreffen sind, geschlossen werden. 


\subsubsection{Anonymität}

Unwissen geht aus der folgenden Interviewsequenz in dreifacher Hinsicht als zentraler Nachteil von Social-Media-Analysen als Methode hervor.

Befragter: Die Gre/, ja. Die Grenzen sind einmal, dass wir, wie gesagt, wir wissen nicht, wen wir befragen. Oder, wir befragen ja nicht mal, sondern jemand gibt eine Antwort von sich aus (Lachen) und, äh, wir reißen diese Antwort aus dem Zusammenhang und zählen sie für bestimmte, äh, Fragestellungen dazu. Das ist schon mal das Erste. Das Zweite ist, wir wissen nie wer dahinter steckt. Wie sieht die Stichprobe aus? Ist er tatsächlich Kunde oder ist er nur jemand, der seine Meinung kundtut? Das Dritte ist, wir wissen gar nicht wie häufig er da berücksichtigt wurde in dieser Social-MediaAnalyse. Ein N von 1 kann dann plötzlich in der Befragung, kann dann plötzlich ein $\mathrm{N}$ von 50 ausmachen. Und das nicht nur bei einem, sondern bei ganz vielen. Dann, wenn wir nach was fragen, dann fragen wir gezielt nach etwas. Bei der Social-MediaAnalyse ist dieses gezielte Fragen ja gar nicht möglich. Und es kommen so Effekte zustande, dass Themen hoch diskutiert werden, die den Kunden eigentlich sonst gar nicht so beeinflussen würden. Und Leute, die in diesen Social-Media- Themen oder auf diesen Seiten unterwegs sind, sind gar nicht unbedingt die Leute, die grundsätzlich die [Kunden der Marke des Unternehmens des Befragten] sind (I2/ S10/ Z13-25).

Erstens besteht Unwissen im Hinblick auf den Kontext oder Zusammenhang, in dem die Inhalte sozialer Medien entstanden sind. Zweitens geht es um Unwissen hinsichtlich der Gewichtung einzelner Inhalte sozialer Medien und damit ihrer Relevanz in einer Social-Media-Analyse beziehungsweise darum, dass Unwissen in Bezug darauf besteht, wie häufig derselbe Sender hinter verschiedenen Inhalten sozialer Medien steckt. Drittens wird Unwissen bezüglich der Nutzer sozialer Medien und damit Sender der in diesen enthaltenen Botschaften deklariert. Den dritten Aspekt behandelt der hier vorliegende Abschnitt als Anonymität. Den zweiten Aspekt greift diese Arbeit im nächsten Abschnitt auf. Der erste Aspekt ist die für diese Studie bedeutsamste Facette von Unwissen, da sie die anderen beiden Aspekte bedingt und all ihre weiteren Ergebnisse durchwirkt.

Im Zuge ihrer Bewertung von Monitoring und Listening sprechen die Befragten davon, dass die Verfahren nicht offenlegen, welche Nutzer hinter einzelnen Inhalten sozialer Medien stehen. Die Identität der Nutzer sozialer Medien sei unbekannt, sie blieben in der Regel anonym. Zwar würden manche Inhalte, die über Social-Media-Analysen untersucht werden und damit Eingang in die Ergebnisse der Verfahren finden, Hinweise darauf enthalten, wer sich hinter den Botschaften verbirgt, doch herrsche in Bezug auf diese Frage keine Gewissheit. Man könne sich auf die Inhalte sozialer Medien nicht verlassen. Aufgrund dessen, dass über Monitoring und Listening nicht ermittelt werden kann, wer hinter den untersuchten Inhalten steckt, beklagen die Befragten ferner, dass sie keine Kenntnis darüber haben, in welchem Kontext die Inhalte entstanden sind oder diskutiert werden und wie häufig 
dieselben Nutzer ihre Botschaft oder weitere, die sich inhaltlich unterscheiden können, in sozialen Medien platziert haben. Daher tritt im Untersuchungsmaterial das Unwissen bezüglich der Identität der Nutzer sozialer Medien beziehungsweise deren Anonymität als zentraler Nachteil der Methode hervor.

Eine weitere Facette des als „Anonymität“ titulierten methodischen Nachteils besteht darin, dass Social-Media-Analysen keinen Aufschluss darüber geben, ob die in sozialen Medien veröffentlichten Inhalte, die unmittelbare Rückmeldungen an das Unternehmen sein können, von Kunden oder Interessenten stammen oder aber von Nutzern, die weder bisher dessen Produkte oder Dienstleistungen gekauft haben noch zukünftig an deren Erwerb interessiert sind. Die Motivation, welche den Inhalten zugrunde liegt, könne heterogen sein. Doch für die Mehrheit der Fragestellungen, die die Automobilindustrie bewegen, sei die Information relevant, auf welcher Art von Erfahrung die zu ihrer Beantwortung ermittelten Ergebnisse beruhen. Nur bei Kunden und Interessenten sei nämlich davon auszugehen, dass sie die Produkte und Dienstleistungen selbst erprobt haben. Diese Frage bliebe bei Monitoring und Listening ebenso unbeantwortet wie die nach der Motivation der Social-Media-Nutzer sich zu Wort zu melden.

Die Befragten merken ferner an, dass Social-Media-Analysen unter Rückgriff auf ,eine eigene Stichprobe“ durchgeführt werden; diese würde nicht direkt der Zielgruppe ihres Unternehmens entsprechen. Die Methode, so wird vermutet, erreicht die Mehrheit der Kunden überhaupt nicht. Der Grund für diese Annahme ist, dass die Befragten ihrem Unternehmen eine „konservative Kundenstruktur“ zuschreiben und davon ausgehen, dass ihre Kunden keine Affinität zu sozialen Medien haben, in diesen also auch nicht aktiv sind:

\footnotetext{
Befragter: Mhm (bejahend). Also auf der einen Seite sind wir zwangsläufig ja unterwegs in einem Umfeld, in dem Menschen, die von sich aus Dinge ins Netz berichten, dieses einfach tun. Das heißt, wir haben hier zwangsläufig (stockt) eine eigene Stichprobe. Es ist mittlerweile recht üblich das zu tun, deswegen kann man sagen, „okay, es ist vielleicht keine massive Verzerrung“, ähm, aber wir erreichen einen großen und (stockt) bei unserer doch recht konservativen Kundenstruktur durchaus bedeutsamen Teil der Kunden NICHT. Das heißt, man muss sich wirklich (stockt) b/, ähm, immer wieder vor Augen führen, dass das (stockt) eine gegebenenfalls eine kleine Teilmenge der Kunden ist. Ähm, und das ist, glaube ich, auch die größte Einschränkung, (stockt) die ich sehe. Es gibt mir einfach kein, ähm, keinen Zugriff auf einen recht bedeutsamen Teil der Kunden (I32/ S17/ Z5-14).
}

Ein weiterer Aspekt des als „Anonymität“ bezeichneten Nachteils von Monitoring und Listening bestehe darin, dass keine genauen Informationen über die Nutzer, die sich in sozialen Medien äußern, gewonnen werden. Der „Detaillierungsgrad“ der Ergebnisse sei zu gering, um diese im Unternehmen weiter zu verfolgen. Manche Befragten treten mit dem Anspruch an Social-Media-Analysen heran, 
über die Methode nicht nur zu erfahren, ob es Kunden ihres Unternehmens sind, die die ermittelten Botschaften auf Social-Media-Kanälen platziert haben, sondern ferner noch deren soziodemographische Daten und sogar die Fahrgestellnummer deren Fahrzeugs zu erhalten.

Ferner wird das Thema „Bots“ im Kontext der Defizite von Social-MediaAnalysen genannt und zu dem als „Anonymität“ bezeichneten Nachteil gezählt. Man wisse bei Monitoring und Listening nie, wie viele Bots hinter einer Veröffentlichung in sozialen Medien oder deren Verbreitung stecken. Das Risiko von Bots müsse stets einkalkuliert werden. Somit blieben bei Social-Media-Analysen nicht nur die Identitäten der Nutzer sozialer Medien anonym, sondern es bliebe auch unklar, ob es sich dabei überhaupt um ,reale“, „menschliche“ Identitäten handelt oder aber um Bots.

Die Schwierigkeiten, die die Befragten hinsichtlich der Interpretation der über Social-Media-Analysen generierten Ergebnisse nennen, wurden bereits angesprochen. Wegen der mit sozialen Medien einhergehenden Anonymität empfinden es die Praktiker als schwierig, die Ergebnisse von Social-Media-Analysen einzuordnen. Nur unter Einbezug des Kontexts, in dem die einzelnen Botschaften stehen, könnten Rückschlüsse auf die Motivation gezogen werden, aufgrund derer die Nutzer sozialer Medien diese formuliert haben. Damit wird die Relevanz des Kontexts der Inhalte sozialer Medien betont. Oft würde dieser bei SocialMedia-Analysen nicht berücksichtigt und in die Ergebnisse einbezogen. Daher könnten die Ergebnisse falsch ausfallen und seien als Grundlage für strategische Entscheidungen ungeeignet. Die Abhängigkeit der Ergebnisse von externen Einflussfaktoren, wie dem Entstehungskontext der Inhalte, betrachten die Befragten als wesentliches Defizit der Methode.

Die Anonymität gilt den Befragten also als ein erheblicher Nachteil von Social-Media-Analysen und bedingt die Bewertung der Methode. Mit der starken Thematisierung des Unwissens in Bezug auf die Nutzer sozialer Medien und deren Anonymität als zentraler methodischer Nachteil tritt zugleich der Wunsch des Unternehmens nach ,gläsernen Kunden' hervor. Kundendaten oder eben Kundenwissen scheinen das höchste Ziel des deutschen Automobilherstellers zu sein. Mit Unwissen hingegen, gleich welcher Art, scheint das Unternehmen nicht umgehen zu können. 


\subsubsection{Unklare Themenrelevanz}

Bei der mit Social-Media-Analysen einhergehenden Anonymität handelt es sich laut den befragten Praktikern um ein Defizit der Methode, das weitere mit sich bringt. So wird auch die unklare Themenrelevanz als Nachteil von Monitoring und Listening kritisiert:

Befragter: [. . . ] Also und das ist, meines Erachtens, der ganze Knackpunkt irgendwie bei dieser Social-Media-Analyse, herauszufinden, A was sind das für Menschen und B wie gravierend sind diese Themen, die da diskutiert werden? Denn selbst wenn ein Thema, ich/ sagen wir mal, innerhalb eines Threads, äh, ewig lang und von vielen Leuten diskutiert wird, ähm, kann es aber trotzdem am Ende des Tages ein, äh, kleiner Baustein irgendwie sein, der, äh, wirklich jetzt nicht wahnsinnig ins Gewicht fällt, ja. Also, sagen wir mal, so diese Einordnung, ähm, ist eben einfach, äh, das ist mein Kenntnisstand, nach wie vor schwierig, was Social-Media-Analyse angeht und birgt damit eben auch gewisse Gefahren der Fehlinterpretation (I7/ S2/ Z36-43).

An der Methode der Social-Media-Analysen bemängeln die praktischen Anwender mit Nachdruck, dass sie die Relevanz von Themen nicht ermittelt. Es würden aus den Inhalten sozialer Medien zwar Ergebnisse gewonnen, doch welche Bedeutung diesen beizumessen ist, vermöge die Methode nicht zu erkennen. Ergebnisse von Monitoring und Listening könnten in Bezug auf ihre Relevanz, Bedeutung oder Gewichtung, auch im Vergleich zu anderen Themen, nicht eingeordnet werden. In dieser Hinsicht blieben bei Social-Media-Analysen Unklarheiten bestehen. Mit der Frage nach der Relevanz eines Themas verbinden die Praktiker die Frage nach dessen Glaubwürdigkeit:

Befragter: [ . . ] Aber es wirft natürlich dann auch wiederum die Fragen der Relevanz und der Glaubwürdigkeit des Ganzen halt auf, weil es ist natürlich nicht ganz frei von/, also du kannst natürlich auch solche Kommentare sehr gut beeinflussen (I14/ S4/ Z2-5).

Ziehen die Befragten jedoch die Ergebnisse von Social-Media-Analysen in Zweifel, so tun sie dies gewissermaßen zugleich mit der Methode als solchen. Monitoring und Listening sind nicht fähig, so betonen sie, eine ,Garantie“ in Bezug auf die Relevanz eines Themas und dessen Einordnung zu geben. Selbst wenn Ergebnisse den Anschein einer Gewichtung verschiedener Themen erwecken würden, sei kein Verlass auf die Bedeutung, die diesen zugewiesen ist. Dies begründen die praktischen Anwender damit, dass die Ergebnisse beeinflusst sein können, etwa durch den Entstehungskontext der Inhalte von Social-MediaPlattformen. Eine Verfälschung sei nicht auszuschließen. Die Ergebnisse, die Social-Media-Analysen generieren, blieben stets „fragwürdig“; eine sichere Einordnung dieser sei unmöglich. Somit werde anhand der Methode zum einen 
nicht ersichtlich, welche Bedeutung einem bestimmten Thema beizumessen, wie dieses zu gewichten oder von welcher Relevanz es ist; zum anderen gehe aus Social-Media-Analysen nicht unmittelbar hervor, welche Themen oder Ergebnisse gegenüber anderen zu priorisieren sind. Dabei handle es sich jedoch um Informationen, die für den Umgang mit den Ergebnissen im Unternehmen und deren Weiterverwendung ausschlaggebend sind.

Ein Grund dafür, dass die Praktiker die Unklarheit in Bezug auf die Themenrelevanz als zentralen Nachteil von Social-Media-Analysen herausstreichen, scheint die mangelnde Quantifizierbarkeit ihrer Ergebnisse zu sein. Die Befragten merken nämlich zudem an, dass die Verfahren keine automatisch quantifizierten Ergebnisse liefern. Quantitative Ergebnisse würden die Relevanz von Themen ersichtlich und diese damit vergleichbar machen. In dem betrachteten Unternehmen wird die Belastbarkeit der Ergebnisse von Social-Media-Analysen direkt mit deren Quantifizierbarkeit in einen Zusammenhang gebracht. Zumindest zeichnet sich anhand des untersuchten Datenmaterials ab, dass der Automobilhersteller nur quantitative oder aber quantifizierte Ergebnisse als „,belastbar“ einstuft. Von der Belastbarkeit der Ergebnisse wiederum, so geht aus den Berichten der Praktiker hervor, ist es abhängig, ob damit gearbeitet wird.

\subsubsection{Mangelnde Informationstiefe}

Im Zuge der Thematisierung der Defizite von Social-Media-Analysen ist als weiterer Nachteil der Methode die mangelnde Informationstiefe anzuführen, in der die befragten Social-Media-Analysten ihren schwerwiegendsten Mangel sehen:

Befragter: [. . . ] Aber, ähm, (...) was teilweise halt schwierig ist, dass (stockt) die Kürze (stockt) der Nachrichten, die Informationstiefe quasi, äh, ausschließt. Also, (...) da wird dann irgendwie, pft, kommt dann so eine Frage, zum Beispiel, äh, ,[Marke des Unternehmens des Befragten, Wettbewerber II, Wettbewerber I], Entscheidung Fragezeichen", äh, und dann muss man quasi interpretieren, was damit gemeint ist (I31/ S7/ Z29-33).

Nach Ansicht der Praktiker steht der Mangel an Informationstiefe in unmittelbarem Zusammenhang mit der in sozialen Medien üblichen Sprache. Diese zeichne sich durch ihre Kürze aus, sei ausgesprochen ,plakativ“. Das führe dazu, dass Inhalte nicht direkt übernommen werden können, sondern der Deutung bedürfen. Ohne Interpretation ist es laut den Social-Media-Analysten kaum möglich, die Botschaften sozialer Medien zu verstehen. Zahlreichen Argumenten, die in Social Media platziert werden, mangle es an Begründungen und Beispielen, anhand derer die Nutzer sie belegen und konkretisieren. Die praktischen Anwender der 
Methode beklagen daher, dass es den Inhalten sozialer Medien an Informationstiefe fehlt. Die ,hinter' einer Botschaft stehende Bedeutung sei oft unklar. Meist sei es nötig, die kurzen Inhalte sozialer Medien zu deuten. Wegen der Notwendigkeit einer Interpretationsleistung sprechen etliche Praktiker der Methode das Potential ab, einen unmittelbaren Erkenntnisgewinn zu liefern.

Vielmehr noch wird im Untersuchungsmaterial auch dargelegt, dass die Mehrheit der über Monitoring und Listening ermittelten Inhalte und damit auch die Ergebnisse der Verfahren nicht nur keine Tiefe haben, sondern fast überhaupt keinen Gehalt aufweisen:

Befragter: //Ja. (Stockt) es kommt einfach 98 Prozent Ramsch. (...) Und dieser Ramsch ist mein Problem. Ich kann eigentlich bloß sagen, ähm, da/ (stockt) das und das Auto wird so und so oft, äh, genannt und in den meisten Fällen positiv und ein paar beschweren sich, dass, äh, dass nach 15 Jahren (stockt) irgendwas rostet. (Stockt) aber das ist eigentlich alles, was ich sagen kann, weil (stockt) die Tiefe einfach nicht gegeben ist (I19/ S14/ Z31-35).

Eine potentielle Inhaltsleere der Ergebnisse von Untersuchungen wird also erstens mit der Methode selbst begründet, zweitens mit der Kürze der in sozialen Medien üblichen Sprache und drittens damit, dass in sozialen Medien in erster Linie Unfug veröffentlicht werde. Darüber hinaus offenbart das untersuchte Datenmaterial noch einen Mangel an ,Sozialforschungskompetenz' und Reflexionsfähigkeit auf Seiten der Befragten.

Monitoring und Listening würden keine Informationen generieren, die den benötigten „Detaillierungsgrad“ aufweisen. Etliche Themen, die durch SocialMedia-Analysen die Aufmerksamkeit der Praktiker erlangten, blieben vage. Informationen, die über die Methode, einen Weg in das Unternehmen , hineinfinden", seien nicht konkret genug, um dort auch bearbeitet werden zu können. In sozialen Medien fänden sich zwar relevante Themen, jedoch seien diese nicht ausreichend ,detailliert“. Befunde, die aus Social-Media-Analysen hervorgehen, könnten oft nicht erklärt und daher auch nicht verstanden werden. Monitoring und Listening sind, so denken die Praktiker, eher dazu geeignet, Ideen zu generieren oder Stimmungsbilder nachzuzeichnen:

Interviewer: Ähm, welche Negativerfahrungen haben Sie gemacht mit Social-MediaAnalysen?

Befragter: Ja, eben, dass es, ah, (stockt) zwar ein Stimmungsbild wiedergibt, aber zu wenig konkrete Daten eigentlich dahinter steckt, um, zumindest was/ was unsere Intention war, wir wollten Probleme möglichst früh erkennen oder Konzeptschwächen möglichst früh erkennen (klopft auf den Tisch). Ah, auf einem seh/ relativ detaillierter Ebene. Und dafür ist es in der Regel zu unkonkret (I21/ S10/ Z18-25). 
Von manchen Befragten wird zudem die Aussagekraft von Tonalitätsanalysen in Frage gestellt. Dieser Zweifel an den in der Regel bei Monitoring und Listening enthaltenen Verfahren ist ebenso Kritik an der Informationstiefe von Social-Media-Analysen.

Da es Social-Media-Analysen an Informationstiefe mangelt, wird den Ergebnissen der Methode auch die Eignung als Basis für strategische Entscheidungen abgesprochen. Der bisherige Verlauf dieser Arbeit hat gezeigt, dass die Social-MediaAnalysten vor allem quantitative oder quantifizierte Ergebnisse als Grundlage für Strategieentscheidungen erwarten. Ergebnisse, die dafür genutzt werden, müssen einen Erkenntnisgewinn liefern, der auf Informationstiefe beruht. Quantitativ ausgerichtete Social-Media-Analysen oder deren quantifizierte Ergebnisse leisten dies nicht und werden daher den Erwartungen des Automobilherstellers nicht gerecht.

Ein weiterer Aspekt von Social-Media-Analysen, der dem methodischen Nachteil einer mangelnden Informationstiefe zuzurechnen ist, besteht darin, dass Monitoring und Listening keine Rückfragen an die Nutzer ermöglichen, die die erhobenen Botschaften in sozialen Medien platziert haben. Diese bei der Methode nicht gegebene Möglichkeit Rückfragen zu stellen trägt nach Meinung ihrer praktischen Anwender auch wieder dazu bei, dass Verständnisprobleme in Bezug auf die in sozialen Medien enthaltenen Inhalte entstehen. Verständnisprobleme könnten sowohl hinsichtlich der verwendeten Sprache und Ausdrucksweise als auch bezüglich der ,hinter' den Inhalten stehenden Botschaft, also deren Bedeutung, nicht gelöst werden.

Der aufgrund mangelnder Informationstiefe fehlende Erkenntnisgewinn ist für die Social-Media-Analysten des betrachteten Unternehmens der bedeutendste Nachteil der Methode. Dieser steht, so zeigt das Untersuchungsmaterial, in direktem Zusammenhang mit den anderen zuvor thematisierten Defiziten von Monitoring und Listening.

\subsubsection{Zwischenfazit:,Weiße Flecken' trotz "quick Wins“" via "quick Shots"}

Social-Media-Analysen zeichnen sich als Methode durch verschiedene ihr inhärente Charakteristika aus; diese bedingen es, dass für die befragten Praktiker des deutschen Automobilherstellers Social-Media-Analysen mit methodischen Vorund Nachteilen behaftet sind. Letztlich beruhen alle methodischen Spezifika der Methode auf der ,Subjektivität ' der Meinungen, Anliegen, Bedürfnisse und Wünsche der Nutzer sozialer Medien, aber auch den Kunden- oder Nutzermotiven sowie deren Dispositionen in sozialen Medien. Aus dieser ,Subjektivität‘ ergibt 
sich der wesentliche Zweck, den die praktischen Anwender des betrachteten Unternehmens der Methode zuschreiben: Eine ,Objektivierung der Subjektivität', welche den Inhalten sozialer Medien anhaftet und soziale Medien als solche auszeichnet. Eine zusammenfassende Bewertung der Social-Media-Analysten in Bezug auf ihre Methode lautet: ,Weiße Flecken“ trotz „quick Wins“ via „quick Shots“. Was darunter zu verstehen ist, klärt dieses Zwischenfazit.

Es wurden unterschiedliche Aspekte der Methode, die die Befragten als Vorteile von Social-Media-Analysen ansehen, thematisiert; diese bedingen sich wechselseitig und tragen wiederum dazu bei, dass die Methode als ein geeigneter Ansatz zur Ermittlung von quantitativen wie , qualitativen', internationalen wie unmittelbaren Erkenntnissen gilt, die ihr Unternehmen in ,Echtzeit' erreichen und die zudem noch aufwandsarm und kostengünstig generiert werden. Zusammengefasst nehmen die Praktiker Social-Media-Analysen als eine Methode wahr, welche „quick Wins “ via „quick Shots“ liefert. Dieses Verständnis der Verfahren des Monitorings und Listenings offenbart sich insbesondere in einer Interviewsequenz, in der ein praktischer Anwender die Vorteile der Verfahren prägnant umreißt und diese ferner als „,nicht-teilnehmendes Beobachtungsverfahren“ bezeichnet. Bedeutsam ist die Interviewpassage auch, da sie auf sozialwissenschaftliche Methodenkenntnisse des sich äußernden Social-Media-Analysten hindeutet. Wie zuvor thematisiert, sind diese im untersuchten Datenmaterial nur selten zu entdecken:

Befragter: (Atmet ein) ja, ein Kosten- und Zeitproblem kann gelindert werden, was wir mit Marktforschung eben immer haben. Ähm, je nachdem, äh, um was es sich handelt, äh, wenn wir relativ viele Teilnehmer haben, die sich zu einem Thema äußern, (atmet ein) äh, ist es halt nicht sehr schwer, Ergebnisse, ähm, in relativ kurzer Zeit und zu überschaubaren Kosten zu bekommen. (Atmet ein) ähm, selbst wenn wir davon ausgehen, dass wir nicht automatisiert, äh, vercoden, was ich, wie gesagt, beim derzeitigen Stand der Technik und auch für den in naher Zukunft zu erwartenden Stand der Technik (atmet ein) für alles andere als empfehlenswert halte. Wir haben dann natürlich einige Vorteile, die sich, äh, daraus ergeben, dass wir es ja im Prinzip mit einem Beobachtungsverfahren zu tun haben, einer nicht-teilnehmenden Beobachtung. Das heißt, wir haben keine Reaktivitäts-Probleme, wir haben sehr schnellen, ähm, Zugriff, äh, auf auch internationale Aspekte, wobei man da sagen muss, dass natürlich der Zugriff auf China sowohl sprachliche als auch Reglementierungs- ähm, Einschränkungen beinhaltet, die schon auch, ähm, zu bedenken sind, und, äh, dass es dann da zu Problemen kommt, äh, die nicht zu unterschätzen sind. Äh, und, ähm, äh, wie gesagt, dass wir auf diese Weise, äh, auch auf einer RELATIV GROSSEN Zahlenbasis qualitative Analysen machen können. Etwas, was ja einfach aus Aufwand- und Kostengründen mit (stockt) klassischen Methoden kaum möglich ist. (Atmet ein) also wir haben hier die Möglichkeit Inhaltsanalysen zumindest teilautomatisiert zu machen, in denen nach Schlagwörtern gesucht wird, (atmet ein) ähm, und dabei eine solche Fülle von Quellen verwenden zu können, die, ähm, mit klassischen Beobachtungsoder Inhaltsanalysen-Fragen ja nie vorliegen (I18/ S6/ Z16-35). 
Die ,hinter ${ }^{6}$ den einzelnen Botschaften stehende Motivation der Nutzer sozialer Medien und der Entstehungskontext ihrer Inhalte sind oft nicht zu erklären und bleiben daher meist unklar, so betonen die Praktiker in Bezug auf die Nachteile von Social-Media-Analysen. Das Bisherige lässt sich mit dem folgenden Befund resümieren: Die Social-Media-Analysten des deutschen Automobilherstellers sind offenkundig nicht dazu in der Lage, mehr an Erkenntnis aus den mannigfachen Aussagen der Nutzer sozialer Medien zu ziehen als explizit verwertbare Hinweise. Sie scheuen davor zurück, nach den Motiven zu fragen, die ,hinter ${ }^{6}$ den Aussagen liegen. Das Misstrauen gegenüber Deutungen, welche sich möglicherweise nicht auch durch zahlenmäßig aggregierte Kernaussagen ,beweisen ' lassen, scheint hoch zu sein. Zugleich gestehen die Befragten ihr Unbehagen gegenüber dieser Art der verkürzenden Interpretation ein, da offenbar irgendwelche Ursachen ,dahinter' stecken. Der herrschende Diskurs im Unternehmen scheint zu verhindern, dass dort Ressourcen wie Arbeitszeit ,verschwendet' werden, wo es darum geht, die möglichen Anliegen, Bedürfnisse, Wünsche der Nutzer sozialer Medien, aber auch die Kunden- oder Nutzermotive sowie deren Dispositionen zu verstehen. Der deutsche Automobilhersteller ,denkt ' fakten- und damit immer auch vergangenheitsorientiert und affirmativ. Ein Durchspielen von alternativen Deutungen und Interpretationen, welche Hypothesen im Hinblick auf ein in der Zukunft liegendes Handeln generieren, scheint im Unternehmen unerwünscht zu sein:

Interviewer: Was sind denn dann für Sie die Grenzen von Social-Media-Analysen, im Sinne von, was können Social-Media-Analysen nicht?

Befragter: (Atmet aus) (...) bei v/ einigen Fragestellungen, glaube ich, können sie (stockt) die Motivation dahinter nicht erklären. Oder das ist dann immer einfach Interpretations-Sache. Also man kann sozusagen, ähm, Symptome betrachten, die Ursache dahinter ist aber dann eigentlich (stockt) an vielen Stellen nochmal offen. Also wenn ich, äh, klar, (stockt) eine konkrete technische Fragestellung analysiere, dann schon, aber wenn ich jetzt sage, ,wieso hat denn das in der Kommunikation gut oder schlecht funktioniert?“, dann kann man sagen, ,okay, lag daran oder daran“, ähm, da wirklich aber, sage ich mal, die Motivation dahinter, warum die Zusammenhänge so sind, glaube ich, wissen wir vielfach einfach nicht. Also wir sehen ja auch dann, (atmet ein) bei uns im Website Tracking einfach Phänomene, wo man sagt, „pft, ist so“. Wir haben halt (stockt) letzten Montag irgendwie doppelt so viel Traffic gehabt wie sonst, wir haben keine Kampagne draußen, wir können es nicht erklären. Und auch, wenn wir dann eben, (atmet ein) Symptome identifizieren, warum das Ganze dann so ist und warum (atmet aus) einem sich dann (stockt) eine Meinung so bildet, ähm, da, glaube ich, hört es relativ schnell auf (I30/ S10/ Z1-13). 
,Weiße Flecken' im Sinne von unbekanntem und unerforschtem Terrain bleiben damit bei jeder Social-Media-Analyse bestehen. Die Methode ist, so befinden die Praktiker, nicht dazu geeignet die ,Welt sozialer Medien' zu erklären. ,Subjektiven' Deutungen und Interpretationen der durch ,Subjektivität' geprägten Inhalte sozialer Medien haftet stets eine auf Unwissen beruhende Unsicherheit an. Monitoring oder Listening liefern zwar mitunter Ergebnisse zu der Fragestellung, wie bestimmte Themen in sozialen Medien bewertet werden, doch fehlt es meist an unmittelbaren Begründungen für diese Bewertungen. In sozialen Medien erkennen die Befragten oft keine Erklärungen dafür, warum Themen auf eine gewisse Art bewertet werden. Die Methode erfordert stets eine Interpretations- und Deutungsleistung. Diese für ein Verständnis der Inhalte von Social-Media-Kanälen unabdingbare Leistung wird jedoch nicht erbracht. Zurückzuführen ist dies auf zwei Umstände: Zum einen mangelt es den befragten praktischen Anwendern der Methode an der für eine Interpretationsarbeit notwendigen Deutungskompetenz. Mit Deutungskompetenz ist hier aber nicht nur die generelle Fähigkeit des Interpretierens von Inhalten sozialer Medien, sondern vielmehr noch die legitime Fähigkeit gemeint. Wie angesprochen, scheint der herrschende Diskurs in dem betrachteten Unternehmen zu verhindern, dass Ressourcen in eine Interpretation von über Social-Media-Analysen gewonnene Ergebnisse fließen, um so Erkenntnisse aus den Anliegen, Bedürfnissen, Wünschen von Kunden sowie Motive und Dispositionen der Nutzer sozialer Medien zu ziehen. Hinzu kommt, dass die Ergebnisse von Social-Media-Analysen oft nicht ernst genommen, sondern lediglich „,belächelt“ werden. Das betrachtete Unternehmen wird von Fakten getrieben; sein Denkstil oder seine ,Logik' ist vergangenheitsorientiert und affirmativ. Eine Deutungskompetenz als legitime und auch anerkannte Fähigkeit des Durchspielens alternativer Interpretationen, beinhaltet und toleriert dieser Denkstil offenbar nicht. Zum anderen - und dieser Umstand beruht auf dem Mangel an Deutungskompetenz - fehlt es dem Unternehmen an der Bereitschaft, sich auf das volle Wissenspotential, das in sozialen Medien liegt, einlassen zu wollen und auch zu dürfen. Dies wird offenkundig nicht nur über die Diskreditierung einzelner Personen ausagiert, sondern auch über die ,Depotentialisierung ' von Social-Media-Analysen als Methode.

Es ist festzuhalten, dass mit Social-Media-Analysen nach Ansicht der Praktiker aus den dargelegten Gründen keine ,Garantie‘ für einen Erkenntnisgewinn verbunden ist. Für die Social-Media-Analysten bleiben die Verfahren des Monitorings und Listenings stets mit einem Risiko behaftet. Als zentraler Nachteil der Methode, der ausschlaggebend für ihre Bewertung ist, kann anhand der Berichte der praktischen Anwender ein Mangel an ,Garantie' auf einen Erkenntnisgewinn rekonstruiert werden. Ergebnissen von Social-Media-Analysen, die als Methode 
„quick Wins“ via „quick Shots“ versprechen, würden stets ,weiße Flecken“ im Sinne von Unbekanntem und Unerforschtem anhaften. Jedoch kann man diesen ,weißen Flecken' nicht anders beikommen als über ihre Deutung. Um die Ergebnisse von Social-Media-Analysen zu interpretieren und so Erkenntnisse mit der Methode zu generieren, ist es unabdingbar, dass das betrachtete Unternehmen eine Deutungskompetenz ausbildet, diese legitimiert und außerdem die Bereitschaft dazu entwickelt, sich auf das gesamte Wissenspotential, welches soziale Medien bereithalten, einlassen zu wollen und vor allem auch zu dürfen.

\subsection{Social-Media-Analysen in Beziehung und im Vergleich}

Zuletzt wurden eine, Depotentialisierung wie Delegitimierung der Methode der Social-Media-Analysen bei dem betrachteten Automobilhersteller dargelegt. Beide Phänomene üben Einfluss auf die Beziehung aus, in welche die Praktiker Monitoring und Listening mit anderen Verfahren der organisationalen Wissensgenese setzen; umgekehrt werden beide Phänomene dadurch noch weiter verstärkt. Die praktischen Anwender diskutieren Social-Media-Analysen in ihrem Verhältnis zu anderen Methoden, insbesondere zu den Verfahren klassischer Marktforschung, und ziehen Vergleiche. Des Weiteren stellen sie in diesem Zusammenhang quantitative und ,qualitative" Verfahren einander gegenüber, wohl mangels fundierter Kenntnisse sozialwissenschaftlicher Forschungsmethoden.

Werden Social-Media-Analysen in Beziehung und im Vergleich diskutiert, so geht es zunächst meist, wie nun dargelegt wird, um die Positionierung der Methode gegenüber klassischer Marktforschung oder auch um das Verhältnis, das beide zueinander haben. Daran anschließend stellen die Social-Media-Analysten in der Regel Bewertungen der Verfahren einander gegenüber, wobei der Fokus auf ihren Vorteilen liegt. Die Nachteile der Marktforschung beleuchten sie kaum; auch deshalb, da diese in ihrem Unternehmen etabliert ist und als nahezu ,unantastbar' gilt. Demgegenüber werden die Nachteile von Social-Media-Analysen hingegen deutlich benannt. Dieser Abschnitt behandelt sowohl die Potentiale von Social-Media-Analysen im Kontrast zu anderen Methoden als auch das Vermögen der Marktforschung Wissen zu produzieren; ebenso nimmt er die Herausforderungen in den Blick, welche Monitoring und Listening im Gegensatz zu anderen Methoden bieten. Die Befragten offenbaren eine Furcht vor der Methode der Social-Media-Analysen. Diese Furcht bedingt es, dass Monitoring und Listening sowie die Ergebnisse der Verfahren bei dem Automobilhersteller nicht für sich alleine stehen dürfen; auch darauf wird eingegangen. 
Die Bewertung von Social-Media-Analysen seitens der praktischen Anwender wurde zwar schon dargelegt, doch werden gewisse Vor- und Nachteile, die die Social-Media-Analysten anderen Methoden gegenüber betonen, erneut aufgegriffen. Social-Media-Analysen in ihrer Beziehung und in einem Vergleich zu anderen Verfahren darzustellen, lässt einerseits erkennen, wie in dem betrachteten Unternehmen verschiedene Methoden der organisationalen Wissensgenese kontrastiert werden, und vertieft andererseits den Befund einer ,Depotentialisierung “ von Social-Media-Analysen.

\subsubsection{Social-Media-Analysen versus Marktforschung}

Social-Media-Analysen versus Marktforschung diskutieren die Praktiker entweder selbst und durchaus hitzig oder sie stellen die Methode als heiß diskutiertes Thema in ihrem Unternehmen dar. Ein wesentlicher Unterschied zwischen SocialMedia-Analysen und Marktforschung besteht darin, dass sich Erstere vor allem durch ihren explorativen Charakter auszeichnen, Letztere hingegen durch ihre Repräsentativität. Da das betrachtete Unternehmen vor allem faktenorientiert ,denkt', gilt Repräsentativität als das entscheidende Gütekriterium. Daher werden auch Marktforschungsstudien, die auf einer großen Grundgesamtheit beruhend repräsentative Ergebnisse zutage fördern, gemeinhin positiv bewertet. Eine derart positive in ihrer Repräsentativität begründete Einschätzung von Marktforschung generell, wie diese in dem Unternehmen zu erkennen ist, bringt es mit sich, dass die durch klassische Marktforschungsstudien gewonnenen Erkenntnisse im Vergleich zu Ergebnissen von Social-Media-Analysen als ,belastbarer“ beschrieben werden.

Zwar hätten auch Marktforschungsstudien Defizite, doch Marktforschung wird eine „Kunst“ genannt. Diese bestehe darin, repräsentative und zugleich valide Ergebnisse hervorzubringen. Marktforschung sei ,nachvollziehbarer“ als SocialMedia-Analysen und daher sowie wegen ihrer großen Grundgesamtheit auch „,belastbarer“; sie ist in dem betrachteten Unternehmen etabliert. Es zeigt sich deutlich, dass dort Marktforschung im Gegensatz zu Social-Media-Analysen legimitiert ist und in deren Anerkennung auch die Bereitschaft des deutschen Automobilherstellers gründet, sich auf ihre Ergebnisse einzulassen. Die SocialMedia-Analysten vermitteln zum Teil gar den Eindruck, dass einzig und alleine quantitative Befragungen den ,methodischen Taktstock' schwingen. Marktforschung unterliegt, so zeigt das Untersuchungsmaterial, der ,Macht der Zahlen“; die Repräsentativität von Studien gilt dem deutschen Automobilhersteller als 
über alle andere Gütekriterien erhaben. Unternehmensintern durchgeführte Studien beruhen seit jeher meist auf quantitativen Messungen und entsprechen damit der allgemeinen ,Logik ${ }^{`}$ des Unternehmens. Bei den durch Marktforschung erzielten Ergebnissen handelt es sich dann um reine Deskription, wie sie über standardisierte Befragungen erzielt wird, und damit nicht zuletzt um affirmative Forschung. Analysen müssen in dem Unternehmen quantitativ sein, um als „fundierte" Studien zu gelten. Alternativen zu den Verfahren klassischer Marktforschung wie Social-Media-Analysen scheinen überhaupt nicht zu existieren - den Eindruck erweckt bisweilen das Untersuchungsmaterial. Anders als Marktforschungsstudien werden Social-Media-Analysen als für das Erzielen fundierter Ergebnisse nicht geeignet bewertet. Mit dieser Beurteilung mögen die SocialMedia-Analysten bei den von ihnen selbst umgesetzten Untersuchungen Recht haben. Es ist nämlich davon auszugehen, dass sie ihre Untersuchungen wegen des schon dargelegten Mangels an sozialwissenschaftlicher Methodenkompetenz und damit einhergehend auch eines Mangels an der für explorative Forschung erforderlichen Deutungskompetenz die Social-Media-Analysen nicht so durchführen, wie es mit entsprechenden Fähigkeiten geschehen könnte. Eine Interpretation der Inhalte sozialer Medien und der Ergebnisse der Verfahren ist unabdingbar, um verwertbare Erkenntnisse zu erlangen. Explorative Forschung bedarf der Kompetenz des Deutens. Über eine solche scheint das betrachtete Unternehmen entweder nicht zu verfügen oder sie ist nicht mehr ausgebildet. Grund dafür ist die Vorrangstellung der Marktforschung, die es ebenso wie der Denkstil des Automobilherstellers bedingt, dass Social-Media-Analysen als explorative Methode keine Legitimation erfahren. Ein ,Nicht-Dürfen“ hat hier ein ,Nicht-Können“ zur Folge; Schuld daran hat die Marktforschung oder aber zumindest die Position, die sie in dem betrachteten Unternehmen innehat:

Befragter: Allerdings (räuspert sich), ich selber bin vom Hintergrund her, ähm, Marktforschung, Marktforscherin auch, und, ähm, habe ein sehr zwie-gespaltenes Verhältnis (Lachen) zu Social Media. Ich glaube, dass das schon hilfreich sein kann, wenn man, äh, Hypothesen generieren möchte oder, wenn man aus Marktforschungsstudien Informationen schon gewonnen hat und die dann nochmal verfeinern möchte und sich überlegen möchte, was sind da noch für Punkte, die ich jetzt nochmal genauer detailliert haben möchte, wo die Marktforschung einfach nicht so tief gegangen ist? Dann kann das hilfreich sein, aber, ähm, ansonsten, ähm, sehe ich persönlich jetzt für das Thema Social Media bei uns eher weniger Einsatzmöglichkeiten (I2/ S1/ Z28-35). 


\subsubsection{Potentiale der Marktforschung}

Unternehmensintern erfährt die Marktforschung bei dem deutschen Automobilhersteller eine fast absolute Legitimation. Es stellt sich daher die Frage, was die Marktforschung im Gegensatz zu der neuen, im Unternehmen bislang noch nicht etablierten Methode der Social-Media-Analysen auszeichnet und welche Potentiale der Marktforschung von den Befragten besonders herausgestellt werden.

Wie zuvor schon erwähnt, gilt die Repräsentativität der Marktforschungsstudien als ihr zentrales Gütekriterium. Marktforschung sei repräsentativ im Hinblick auf die für den Automobilhersteller relevante Grundgesamtheit. Zudem sei Marktforschung valide. Die Befragten stellen heraus, dass ihnen die untersuchte Grundgesamtheit bekannt ist. Marktforschungsstudien würden ,aussagekräftige" Ergebnisse hervorbringen und diese könnten aufgrund der spezifischen Eigenschaften von Marktforschung auch zueinander in Bezug gesetzt werden. Verzerrungen von Marktforschungsergebnissen schließen die Praktiker nahezu aus. Was Marktforschung behauptet - so die Annahme - gilt.

Für das Unternehmen ist es weiterhin relevant, dass Untersuchungen ,harte Fakten' generieren. Die ,Härte' von Ergebnissen wird auf deren Repräsentativität zurückgeführt, besteht also in Quantitäten und beruht auf der ,Macht der Zahlen'. Es scheint als würde die ,Härte " von Ergebnissen in einen exklusiven Zusammenhang mit ihrer Belastbarkeit gestellt. Nur ,harte' Ergebnisse hätten das Potential, Aussagekraft zu entfalten und für diese einzustehen. „Zahlen, Daten, Fakten“ sind gefordert. Mit anderen Erkenntnissen kann der Automobilhersteller, so offenbart das Untersuchungsmaterial, mangels der Kompetenz sie zu interpretieren und anhand von Deutungen zu verstehen, nicht umgehen. Berichte der Social-MediaAnalysten ,erhärten' gar den Eindruck, dass ihr Unternehmen ,Garantien“ von empirischen Untersuchungen erwartet und daran die Güte von Studien bemisst. Doch derartige ,Garantien ' beruhen laut den Befragten auf Repräsentativität. Daher erfahren ausschließlich quantitative Analysen, die in der Vergangenheit etabliert wurden, Legitimation.

Es gilt als ,das Qualitätskriterium “ von Marktforschung, dass sie mit großen Zahlen arbeitet und breiter aufgestellt ist als Monitoring und Listening. Marktforschung nehme Segmentierungen vor und erstelle Kreuztabellen. Die Auswertungsverfahren lieferten Informationen des geforderten „Detaillierungsgrads“ und in Bezug auf die ,relevante Grundgesamtheit“. Aufgrund der Repräsentativität der Ergebnisse sowie der Kenntnis der Grundgesamtheit generiere Marktforschung außerdem ,echte“ Details. Anhand von Marktforschung entstandene Ergebnisse 
gelten im Unternehmen als ,verifiziert“. Auch dies ist ein weiterer Hinweis darauf, dass sich die befragten Praktiker nicht näher mit sozialwissenschaftlichen Forschungsmethoden und deren Methodologie beschäftigt haben. Wäre dies der Fall, wären sie wohl der Wissenschaftstheorie des Kritischen Rationalismus von Karl R. Popper (1902-1994) begegnet und würden, aufgrund von dessen Ausführungen über den Falsifikationismus oder die Methode der Falsifikation, mit dem Begriff „Verifikation“ im Kontext des Empirismus gemäßigter jonglieren. ${ }^{18}$ Stattdessen sind die praktischen Anwender der Ansicht, dass klassische Marktforschung für spezifische Fragestellungen geeigneter ist, da sie „verifizierte“ Antworten liefert.

In der Frage, ob Marktforschungsstudien die benötigten Detailinformationen liefern oder zu „oberflächliche“ Ergebnisse generieren, herrscht Uneinigkeit. Einstimmigkeit der Befragten besteht wieder darin, dass Marktforschung erheblich wissenschaftlicher sei als Social-Media-Analysen. Diese Einschätzung kann nur darin begründet sein, dass es den Praktikern an sozialwissenschaftlicher Methodenkompetenz mangelt, vor allem bezüglich ,qualitativer" Verfahren. Vielmehr noch scheint das betrachtete Unternehmen überhaupt kein Bewusstsein für die ,Qualität qualitativer Forschung ' zu haben, deren Güte und Gütekriterien. Zurückzuführen ist dies nicht zuletzt darauf, dass empirische Studien ,qualitativer' Art keine Legitimation in der klassischen, nahezu rein quantitativ ausgerichteten Marktforschung oder vielmehr gegenüber dieser erfahren.

Aufgrund dieser gewohnten Potentiale der Marktforschung lehnen es die Befragten ab, sich auf das gesamte Wissenspotential, welches Social-MediaAnalysen zutage fördern können, einzulassen. Diese ,verweigerte Legitimation “ von Social-Media-Analysen liegt auch in den, mitunter nur vermeintlichen Potentialen der Marktforschung begründet. Es mangelt an Deutungskompetenz. Für explorative Untersuchungen und die Interpretation der Inhalte sozialer Medien und Ergebnisse von Social-Media-Analysen ist diese jedoch unabdingbar. Im Hinblick auf die fehlende Deutungskompetenz ist $\mathrm{zu}$ bedenken, dass diese wegen ihrer, mitunter in den Potentialen der Marktforschung wurzelnden NichtLegitimation in dem betrachteten Unternehmen in ,Vergessenheit ' geraten sein kann.

${ }^{18}$ Bezüglich des von Popper begründeten Kritischen Rationalismus siehe beispielsweise: Böhm, Holweg, \& Hoock, 2002; Hillmann, 2007, S. 467 f.; Niemann, 2004; Popper, 1996/2018, 1987/2016, 1935/1984; Salamun, 1989. 


\subsubsection{Potentiale von Social-Media-Analysen}

Ebenso beschreiben die Befragten etliche Potentiale von Social-Media-Analysen, die die Methode gegenüber anderen auszeichnet. Einzig bei einer Gegenüberstellung mit der Marktforschung entsteht der Eindruck, es gebe keine Alternative dazu. Derzeit werden die Potentiale von Monitoring und Listening noch nicht ausgeschöpft, möglicherweise auch wegen ihrer Bewertung gegenüber den Marktforschungsmethoden. Der Umgang mit den Verfahren erfolgt pragmatisch.

Social-Media-Analysen zeichnen sich gegenüber anderen Methoden nach Ansicht ihrer Praktiker vor allem durch ihren explorativen Charakter aus. Zugleich aber machen diese deutlich, dass explorative Forschung in ihrem Unternehmen nicht legitimiert ist und aus diesem Grund - wenn überhaupt - nur sehr verhalten praktiziert wird:

Befragter: [. . . ] Was wir aber halt besser hinbekommen müssten, wäre dieses/ diese, mhm (überlegend), normalerweise planen wir ja eine Befragung, sei es ein Usability Test im Fahrsimulator oder eine Marktforschungs-Maßnahme, und, ähm, wir müssten uns eigentlich auf dieses andere Modell einlassen, zu sagen, wir schauen/ wir schauen quasi (stockt) im Netz und häufig ist halt so ein Thema, wie soll ich sagen, ein eher saisonales Thema, also, ähm, es kommt eine neue App auf den Markt, die tut irgendwie gar nicht gut bei [Marke des Unternehmens des Befragten] (...) funktionieren und die Kunden sind frustriert und, ähm, ein halbes Jahr später fragen/ befragen wir sie zu ihren Erfahrungen mit Apps und (...) ich weiß dann nicht, wie sich diese Erfahrung auswirkt, die ein halbes Jahr vorher passiert ist. Hat die noch eine Bedeutung, ähm, hat die vielleicht, jetzt gibt es ja Carry-Over-Effekte vielleicht, ähm, (...) und (..) was eigentlich eben spannend wäre, wäre tatsächlich hier stärker am Puls der Zeit zu bleiben und dieses/ (...) Nutzer initiierte Meinungen auf einem schnellen Weg in eine/ in eine systematische Befragung zu überführen, bevor (stockt) dieser Sturm eigentlich vorbei ist. Und so was kann man relativ aufwandsarm eigentlich machen. Ich glaube auch nicht, dass dafür große Mittel erforderlich sind, aber um eine spätere Relevanz (stockt) für [Marke des Unternehmens des Befragten] und die Entscheidungs-Prozesse möglichst früh zu erkennen, wäre es, glaube ich, ganz geschickt (I32/ S15-16/ Z36-3).

Die praktischen Anwender sehen durchaus Potential in Social-Media-Analysen, haben aber Schwierigkeiten, die Methode in der ,quantitativen Logik' ihres Unternehmens zu verorten. Wenn sie ihre Praxis beschreiben, sprechen sie mitunter davon, dass Social-Media-Analysen eine „qualitative“ Methode markieren. Diese Erkenntnis hält sie jedoch weiterhin nicht davon ab, die Verfahren quantitativ ,zu denken“. Dies zeigt sich etwa dann, wenn vom „Messen“ oder „Tracken“ sozialer Medien durch „qualitative“ Social-Media-Analysen die Rede ist oder erhobene Daten nicht qualitativ ausgewertet, sondern ohne jegliche Interpretation quantitativ ausgezählt und in dieser Form dann innerhalb des Unternehmens genutzt 
und damit quasi ,missbraucht" werden. Die Methode kommt unternehmensintern zum Einsatz, um eigene Thesen zu belegen oder zu illustrieren; dies streichen die Befragten als Potential im Vergleich zu anderen Verfahren heraus. Die Praktiker sehen meist davon ab, ,hinter' die Ergebnisse zu blicken. Zwar scheint die Methode wegen ihres explorativen Charakters selbst nicht legitimiert zu sein, wird aber verwendet, um , subjektive Denkweisen zu objektivieren“. Hier offenbart sich abermals die ,Logik' des betrachteten Unternehmens als affirmativ. Da andere Denkstile keine Legitimation erfahren, greift diese ,Logik' auch bei explorativen Untersuchungen.

Jedoch versuchen nicht alle Social-Media-Analysten, Monitoring und Listening, wenn sie über die Potentiale dieser von ihnen als ,qualitativ ‘ eingeordneten Verfahren im Vergleich mit anderen sprechen, in ,quantitative Schubladen ' zu stecken. Die Mehrheit der praktischen Anwender hat wohl erkannt, dass sich Social-Media-Analysen von den etablierten und in ihrem Unternehmen als legitim anerkannten Ansätzen, die auch die Marktforschung nutzt, unterscheidet. Doch scheint es an entsprechendem methodischem Vokabular zu fehlen, um diese Differenzen präzise benennen zu können. Die Praktiker greifen auf Umschreibungen zurück. Dies verdeutlicht erneut den bestehenden Mangel an fundierten Kenntnissen des sozialwissenschaftlichen Methodenkanons. Erwähnt wird nur, dass die Methode nicht für quantitative Untersuchungen geeignet sei, stattdessen die Stimmung der Kunden und Nutzer sozialer Medien wiedergeben könne.

Da es sich bei Social-Media-Analysen nicht um quantitative Untersuchungen handle, sei zu bedenken, dass die dank der Methode ermittelte Stimmung nicht mit den Meinungen der Grundgesamtheit übereinstimmen beziehungsweise deren Verteilung entsprechen müssten. Mit einer solchen Umschreibung verweisen die Befragten darauf, dass Social-Media-Analysen keine repräsentativen Ergebnisse liefern. Trotzdem würden sie auf der großen Zahlenbasis sozialer Medien oder der Netzöffentlichkeit beruhen. Monitoring und Listening würden somit ,qualitative Ergebnisse hervorbringen, die auf einer großen Quantität beruhen. Die Stichprobe bei Social-Media-Analysen sei damit letztlich sogar noch größer als bei Marktforschungsstudien. Dennoch wird den Ergebnissen der Methode innerhalb des Unternehmens die Aussagekraft abgesprochen.

Auszeichnen würde sich die Methode gegenüber anderen dadurch, dass die Verfahren kostengünstig, in hoher Geschwindigkeit und auf simple Art Ergebnisse liefern, die zudem internationale Aspekte einbeziehen. Wie schon gezeigt, werden diese Vorteile von den Befragten detailliert diskutiert. Es handelt sich hier um solche, die anhand von ,harten Fakten' bemessen werden können; Kosten, Zeit, Aufwand und Internationalität können exakt beziffert werden. Anders gestaltet es sich jedoch mit dem Aspekt der „Authentizität“, die die praktischen 
Anwender als methodisches Kennzeichen betonen. Laut eines Befragten ist es der „social Kontext“" der soziale Medien auszeichnet. Ihre Authentizität sei ein besonderes Merkmal von Social-Media-Analysen gegenüber den anderen Methoden; selbst Marktforschung entstehe in künstlichen Laborsituationen. Das Potential von Social-Media-Analysen besteht nach Ansicht der Praktiker in ihrem ,social Kontext". Ergebnisse der Methode würden die ,Realität" und die ,Wirklichkeit" der Nutzer, die Botschaften in sozialen Medien platzieren, abbilden. Über Monitoring und Listening würden „reale“ Fallbeispiele und Situationen von Kunden und Nutzern sozialer Medien und deren Emotion ermittelt. Die Authentizität, die die Social-Media-Analysten ihrer Methode zuschreiben, rekurriert insbesondere darauf, dass es „O-Töne“ oder „Original-Aussagen“ seien, die sie aus der Netzöffentlichkeit gewinnen. „Social“ kann mit „Lebenswirklichkeit“ übersetzt werden, wobei diesen Begriff, der an den der ,Lebenswelt“" erinnert, ein Befragter einführt. Der Einblick in die „Lebenswirklichkeit“ der Kunden und Nutzer sozialer Medien, den Social-Media-Analysen eröffnen, sei das entscheidende Potential der Methode und „qualifiziere" diese gegenüber alternativen Verfahren. Ihre Annahme begründen die Social-Media-Analysten damit, dass bei deren Anwendung nur passiv „zugehört“ oder „,beobachtet“, aber niemals aktiv „nachgefragt“ wird; Reaktivitätsprobleme können daher nicht auftreten. Mit Social-Media-Analysen wird „Lebenswirklichkeit“" anhand von sozialen Medien also explorativ erkundet:

Befragter: [. . . ] Also, wir haben, einfach, äh, eine Innovation beginnt ja irgendwo bei einem Problem. Quasi. Und es ist ein Riesen-Now-Know, das bei den Usern steckt. Wenn ich wüsste, worüber die diskutieren, an ihrem Stammtisch und so weiter, wenn ich dort ein Mikrophon aufstellen könnte und zuhören, dann kriege ich auch viel, viel mehr mit als wenn ich sie befrage, äh, dort zu irgendwas. Habt ihr da ein Problem, ja oder nein? Und, das ist ganz was Anderes als wenn ich es offen mitkriege (I15/ S1/ Z9-14).

Monitoring und Listening messen laut den Befragten „soziale Strömungen“, erfassen die „Lebenswirklichkeit“ der Zielgruppe des Unternehmens und stellen Relationen her. Derart versuchen die Praktiker zu beschreiben, dass Social-MediaAnalysen explorative Verfahren sind und als solche gehandhabt und bewertet werden müssen. Auch diese Einsicht benennen sie nicht entsprechend. Die Methode ist, so streichen sie heraus, dazu imstande, detaillierte Informationen $\mathrm{zu}$ erheben, ein tiefes Verstehen zu ermöglichen, Erklärungen zu bieten und „Neues“ zu entdecken. Social-Media-Analysen würden im Gegensatz zu anderen Verfahren Tendenzen und Trends erkennen, die sonst nicht in das Unternehmen ,hineinfänden“. Für Innovationen sei dies entscheidend. Somit sehen die Social-Media-Analysten durchaus Potentiale in der ,qualitativen“ Forschung. 
Letztere sei für ihr Unternehmen wesentlich, doch es müsse die notwendige Deutungskompetenz erst ausbilden. Diese sei erforderlich, um , qualitative ' Untersuchungen umsetzen, ihre Ergebnisse interpretieren und reflektieren zu können. Laut ihren praktischen Anwendern zeichnen sich die Verfahren dadurch aus, dass sie ,realer“ sind als andere Ansätze, weil näher an der ,Lebenswirklichkeit". Social-Media-Analysen erzeugen ,Transparenz“:

Befragter: [. . . ] Ähm, das ist halt die gewisse Transparenz, die du, äh, ansonsten mit einer klassischen Marktforschung nur sehr teuer hinkriegst. Und auch nicht unbedingt in der Zeit. Also da ist dieses Instrument natürlich extrem überlegen, ähm, und wird deswegen natürlich auch sehr gern genutzt (I14/ S3-4/ Z47-2).

Wird die Methode richtig angewendet, liefert sie gemäß den Befragten tiefergehendere Ergebnisse als andere Verfahren; dies gilt auch in Bezug auf die Marktforschung.

\title{
5.3.4 Herausforderungen von Social-Media-Analysen
}

Neben den Potentialen, die die praktischen Anwender in der Passivität der Methode erkennen, sehen sie ebenso zahlreiche Herausforderungen von SocialMedia-Analysen, insbesondere im Hinblick auf eine Interpretationsleistung und Deutungskompetenz:

\begin{abstract}
Befragter: [. . . ] Und man hat eben auch keine Möglichkeit nachzufragen. Wenn ich ein qualitatives Interview führe und jemand macht ein Statement, wo ich mir nicht sicher bin, was hat er gemeint, habe ich es richtig verstanden, dann kann ich nachfragen. Bei einem inhaltsanalytischen, (atmet ein) äh, Fragestellung nicht. Und nachdem das nun mal, sagen wir mal, RELATIV umgangssprachlich dann ja auch, äh, oft formuliert ist, äh, in den Foren oder den, äh, sozialen Medien, (atmet ein) kann es natürlich sein, dass man wieder vor dem Problem steht, dass man sagt, interessantes Statement, aber ich weiß nicht genau, was er damit jetzt meint. (Atmet ein) ähm, und, äh, das ist, ähm, dann etwas, was die, ähm, Möglichkeiten ein bisschen eingrenzt, äh, aber, wie gesagt, äh, in dem Moment wo man sich klar ist, dass ist nicht für alles einsetzbar, es ersetzt nicht, äh, die gesamte klassische Umfrageforschung, äh, qualitativ wie quantitativ, (atmet ein) ähm, ist es ein sinnvolles Tool (I18/ S8/ Z31-41).
\end{abstract}

Monitoring und Listening sprechen ihre Praktiker entweder die Aussagekraft ab oder sagen ihnen eine geringere als der Marktforschung zu; dennoch konstatieren sie, dass die Verfahren die „Lebenswirklichkeit“ der Kunden und Nutzer sozialer Medien besser abbilden. Auch dies ist wohl auf die Delegitimierung ,qualitativer“ 
Forschung und die damit einhergehende ,Depotentialisierung ' von Social-MediaAnalysen zurückzuführen. Wie schon mehrfach erwähnt, offenkundig passen weder qualitative Methoden noch ein exploratives Vorgehen in die ,Logik' des deutschen Automobilherstellers. So nennen die Social-Media-Analysten zwar einige Potentiale der Methode, merken aber auch an, dass diese im Vergleich zu anderen keine Aussagen in Bezug auf die „schweigende Masse“ generieren kann. Außerdem bieten Monitoring und Listening laut den Befragten keine Möglichkeit „,nachzufragen“. Die ,subjektiven“ durch die Verfahren ermittelten Meinungen müssten „ertragen“ werden, ohne sie verifizieren zu können.

Zwar haben die Praktiker erkannt, dass sowohl die Inhalte sozialer Medien als auch die Ergebnisse von Social-Media-Analysen nach Interpretationen verlangen, es zeigt sich jedoch ebenso, dass qualitative Deutungen in dem betrachteten Unternehmen undenkbar sind. Die Social-Media-Analysten sind nicht fähig, mehr Erkenntnisse aus Inhalten sozialer Medien zu ziehen als explizite Hinweise; auch dann nicht, wenn sie sich der Notwendigkeit des Deutens bewusst sind. Nach Motiven, die ,hinter' den einzelnen Botschaften stecken, wird überhaupt nicht gefragt. Vielmehr besteht ein Misstrauen gegenüber Interpretationen. Die Methode der Social-Media-Analysen gewährt laut den praktischen Anwendern keine ,Garantie" und eben dies wird ihr zum Verhängnis. Ohne eine quantitative ,Erhärtungen', wie die Marktforschung sie generiert, stufen die Social-MediaAnalysten die Ergebnisse als nicht verwertbar ein. In erster Linie geht es dem deutschen Automobilhersteller nicht darum, mögliche Anliegen, Bedürfnisse, Wünsche sowie Kunden- oder Nutzermotive und deren Dispositionen zu verstehen, sondern der eigenen affirmativen, fakten- und vergangenheitsorientierten ,Logik' Rechnung zu tragen. Eine Auseinandersetzung mit alternativen Deutungen, die Hypothesen in Bezug auf in der Zukunft liegendes Handeln generieren, wird innerhalb des Unternehmens offensichtlich nicht gerne gesehen.

Dennoch offenbaren die Praktiker, dass ihnen Social-Media-Analysen als neue Methode nicht vorhandene Fähigkeiten abverlangen. Mit Monitoring und Listening ist plötzlich Deutungskompetenz gefragt, ja sogar gefordert. Ergebnisse von Social-Media-Analysen bedürfen der Interpretation, denn ohne eine solche können die praktischen Anwender nicht mit diesen umgehen. Die Ergebnisse müssen ihrer Einschätzung nach „kalibriert“ und in eine umfassende Betrachtung integriert werden: 
Befragter: [. . . ] Also, die/ also für mich ist Social-Media-Analyse ein Teilaspekt aus einer/, also aus/, das sind verschiedene Blickwinkel auf den Kunden und es müsste ja im Prinzip verschiedene Quellen nehmen. Also einmal habe ich meinetwegen/ ich habe meinetwegen ein CIC. Als was wird denn da gesprochen? Gewährleistung- und Kulanz-Cases als Beispiel mal. Dann gibt es Social Media (klopft auf Tisch). Dann gibt es Marktforschung (klopft auf Tisch). Und dann gibt es bestimmt nochmal andere Quellen, die ich jetzt gerade nicht auf dem Schirm habe. Und man müsste ja zu so einer konsolidierten Sichtweise kommen auf den Kunden und dann das auch ins Verhältnis setzen. Ähm, liegt das vielleicht am Set-up, dass Marktforschung damit nicht so, offen gesprochen vielleicht, wie abgelästert wird im Netz. Ist das wahrhafter oder (stockt) ist bei den Gewährleistung und Kulanz ist es viel zu negativ? Weil, da wollen die Leute Geld haben. Was weiß ich. Also, man müsste es so ein bisschen kalibrieren und aus einer (stockt)/ eine Gesamtsicht vielleicht daraus schmieden. (I12/ S10/ Z6-17).

\subsubsection{Keine „Solonummer"}

Wenn praktische Anwender berichten, dass Social-Media-Analysen nicht alleine zum Einsatz kommen dürfen, meint dies zweierlei. Einerseits betonen sie, dass die Inhalte sozialer Medien in ihrem Kontext zu betrachten sind, andererseits weisen sie darauf hin, dass es die Ergebnisse der Methode mit zusätzlichen Quellen wie etwa Studien der Marktforschung zu verknüpfen gilt. Social-Media-Analysen sind keine „Solonummer“. Deutung und Interpretation müssen in zweifacher Hinsicht erfolgen.

Anders als Methoden, die Rückfragen zulassen, würden Social-MediaAnalysen nach Deutungen verlangen. Nur mit einer Deutungskompetenz, die innerhalb des betrachteten Unternehmens auch legitimiert ist, sind Interpretationen zu leisten. Laut den Befragten müssen die Ergebnisse von Monitoring und Listening „kontextualisiert“, also unter Einbezug ihres jeweiligen Entstehungskontexts interpretiert und in ihrer Deutung mit anderen, zusätzlichen Erkenntnissen in einen Zusammenhang gestellt werden. Da die Social-MediaAnalysten zu einer derartigen Interpretation jedoch derzeit gar nicht in der Lage sind, fällt ihnen auch der Umgang damit schwer. Von anderen Methoden, die sie mit Social-Media-Analysen vergleichen, ist ihnen das Deuten nicht bekannt oder gar vertraut. Wegen der unternehmensintern fehlenden Legitimation für Interpretationen mangelt es ihnen an der Bereitschaft sich auf das Wissenspotential einzulassen, das mit sozialen Medien einhergeht und anhand von Social-MediaAnalysen zutage gefördert werden könnte. Die Praktiker nutzen die Methode nicht für explorative Untersuchungen, wofür sie sich aber vorrangig eignet und auch genutzt werden könnte. Der ,Logik' ihres Unternehmens entsprechend drängen sie die Verfahren stattdessen in Richtung einer affirmativen Forschung. 
Offenkundig beginnen die Befragten aber zu erkennen, dass Monitoring und Listening mehr leisten können als quantitative Deskriptionen, denn sie sprechen ebenso davon, dass ihr Unternehmen ein ,qualitatives Verständnis' entwickeln muss. Social-Media-Analysen könnten neue Perspektiven, wie die „Lebenswirklichkeit“ der Nutzer sozialer Medien oder die der eigenen Kunden eröffnen:

Befragter: [. . . ] Äh, Social Media kann Ihnen helfen, eine Sache nochmal vor der anderen Perspektive her zu betrachten. Wir, äh, sind ja sehr rational unterwegs, äh, wir haben ganz klare Ansprüche an Analysen, (stockt) wie die aufgestellt sein müssen, (stockt) welchen Ansprüchen die genügen müssen. Ähm, und damit, denke ich, klammern wir einen Teil der Welt, der Lebenswirklichkeit aus. So. Und, äh, genau da liegt die Chance für Social Media, diese, wenn es auch nur kleine Räume sind, diese klein ausgeklammerten Teile der Lebenswirklichkeit mit auszuleuchten. Weil da kommt tatsächlich, äh, nochmal, äh, (stockt) ein subjektiver, äh, Betroffenheits-Fakt mit rein, den wir sonst so gar nicht draufhaben. Ja, (stockt) den Sie auch nicht simulieren können. Äh, das ist in der Tat eine Chance, äh, sozusagen, so eine Art, äh, da wo es unklar ist, eine Art Reality Check zu machen. Da (stockt) könnte man auch nochmal eine Wasserstands-Meldung (stockt) abholen. [. . . ] Aber, immer als Additiv zu sehen und nicht als, äh, sozusagen, äh, als erster/ als erster Ansatz (I22/ S8-9/ Z38-18).

Über Social-Media-Analysen würde also ein „Blick in die Realität“ möglich. Auf einen solchen, kann sich der deutsche Automobilhersteller jedoch noch nicht einlassen und schon gar nicht verlassen. Zwar wird wegen ihres explorativen Charakters Potential in Social-Media-Analysen erkannt, doch auch eingestanden, dass es dem Unternehmen an entsprechenden Qualifikationen mangelt und die Methode daher bislang ausschließlich im Zusammenspiel mit Marktforschung und nicht alleine genutzt wird. Wegen ihres explorativen Charakters könnten anhand von Social-Media-Analysen zwar Innovationen entdeckt werden, doch nach Ansicht der praktischen Anwender bildet die Methode den sozialen „IstStand" ab und ist daher vor allem für explorative Ad-hoc-Untersuchungen in Ergänzung zur Marktforschung geeignet. Da ein derartiges exploratives Vorgehen eine Grauzone für den Automobilhersteller markiert, hat dieser bisher keinen Umgang mit Social-Media-Analysen etabliert: 
Befragter: [. . . ] Ja, an dem Prozess letzten Endes sind wir dann halt eben irgendwo stehen geblieben. Also das haben wir dann halt nicht weiter/ nicht weiter vertieft, zumal ja, wie gesagt, auch unterschiedliche Fachabteilungen ein Stück weit ihre eigenen Social-Media-Analysen da so ein bisschen fahren und es nie so ganz klar war, ist es jetzt ein Marktforschungsthema oder ist es jetzt kein Marktforschungsthema oder ist es eine Methode innerhalb der Marktforschung, die wir auch verwenden, aber die andere Fachabteilungen quasi auch verwenden können. Also, das ist so ein bisschen/ so ein bisschen so eine Grauzone zwischen klassischer Marktforschung und zwischen, äh, ich mache halt hier, äh, ähm, nee, vor meiner Kommunikation und, äh, kriege hier ein Feedback. So wie, äh, man auch Kundenfeedback draußen, äh, im Handel bekommt oder so, auf direktem Wege, wo wir ja auch nicht immer den Finger darauf haben und sagen können, das ist jetzt eine Meinung, die zählt oder das ist eine Meinung, die halt nicht zählt (I14/ S1-2/ Z40-4).

In der Unternehmenspraxis markieren Social-Media-Analysen nach wie vor ein ,exploratives Novum‘. Im Hinblick darauf mangelt es sowohl an einer Deutungskompetenz als legitime Fähigkeit als auch an der Bereitschaft sich auf das Wissenspotential von Social-Media-Analysen einlassen zu wollen und zu dürfen. Daher werden Social-Media-Analysen auch als ein plötzlich in Erscheinung tretendes Phänomen wahrgenommen, das sich nicht in die etablierten ,Schubladen“ legitimierter Methoden ,stecken' lässt, diese höchstens ergänzen darf. Einerseits werden Social-Media-Analysen und Marktforschung als Gegensätze gesehen; erstere würden nicht mehr, sondern ähnliche, vor allem aber flachere Ergebnisse als Letztere liefern. Andererseits wird dies gegenteilig wahrgenommen; die Methode gilt als ,ein Muss' und man versucht, sie neben der Marktforschung oder als ihr Gegenüber zu positionieren:

Befragter: Ähm (überlegt), was können Sie nicht? Was können sie nicht? Die Frage ist, ähm, ja, mit der Repräsentativität bin ich mir nicht ganz so, ähm, sicher, natürlich. Sie geben einen, ähm, Einblick in die Gefühlslage oder den BUZZ, wie es ja eben so schön heißt. Also, was wird gerade gesprochen? Insofern/. Und das ist real. Insofern, ähm, muss man es beachten. Es ist einfach eine/, es ist wie ein Pulsmesser, der gerade/ was gerade so passiert. Aber ist das jetzt ein guter Schätzer für den Gesamt-Zustand des Körpers? Da bin ich mir auch wiederum nicht so sicher. Also insofern ist in der Marktforschung vielleicht da, ähm, mit der Zufallsstichproben-Ziehung/. [. . . ] Also, nichtsdestotrotz ist es REAL. FACTUM EST. Also, das ist/, das ist da. Ähm, insofern muss man es beachten, man muss es monitoren, ob es ein guter/, ob es ein Ersatz von einer guten, äh, Marktforschung ist zum Beispiel, da bin ich mir nicht so sicher. Ja (I12/ S7-8/ Z46-10). 
Bei Social-Media-Analysen, so offenbart die Interviewsequenz, handelt es sich um eine Methode, die der Methodenkoffer eines Unternehmens heutzutage zwingend beinhalten sollte. Doch wo die Methode einzusortieren ist, ist noch nicht geklärt. Einerseits sei es denkbar, Ergebnisse von Monitoring und Listening über Marktforschungsstudien zu verifizieren; andererseits seien Social-MediaAnalysen eine Alternative zu klassischer Marktforschung oder auch ein ,Weg um diese herum'. Laut den Befragten ermöglicht es die Methode, selbst Untersuchungen durchzuführen, ohne die für die Marktforschung zuständige Abteilung des Unternehmens einzubeziehen. Diese Ansicht wiederum führt dazu, dass SocialMedia-Analysen auch als Gefährdung der etablierten Marktforschung bewertet werden. Social-Media-Analysen haben in dem betrachteten Unternehmen der deutschen Automobilindustrie noch keine eigene Daseinsberechtigung, dürfen nicht für sich alleine stehen. Daher wird die Methode oft im Hinblick auf den Kontext der Inhalte sozialer Medien oder deren Ergebnisse in einer zu erstellenden Gesamtschau diskutiert.

\subsubsection{Zwischenfazit:„,Puzzlespiel“ und „Mosaiklegen“}

Sofern die Praktiker des deutschen Automobilherstellers Social-Media-Analysen mit anderen Methoden, allen voran mit Methoden der klassischen Marktforschung in eine Beziehung setzen oder einen Vergleich mit diesen ziehen, offenbart sich fast eine Furcht vor dem ,explorativen Novum', als das sie Social-MediaAnalysen einordnen. Wegen dieser Furcht dürfen die Ergebnisse von Monitoring und Listening nicht für sich alleine stehen. Trotzdem nutzt das betrachtet Unternehmen die Ergebnisse der Verfahren für sein „Puzzlespiel“ und „Mosaiklegen“, das dieses Zwischenfazit thematisiert.

Marktforschungsstudien und Social-Media-Analysen werden meist insofern zueinander in Beziehung gesetzt, dass Letztere einen „Mosaikstein“ oder ein „Puzzleteil“ in einer von der Marktforschung generierten Gesamtbetrachtung ausmachen. Die Praktiker der Methode deklarieren Social-Media-Analysen als Zusatz zur Marktforschung oder zu anderen Methoden. Jedoch handelt es sich, so stellen sie klar, um eine Ergänzung, die zwingend erforderlich ist und dennoch im Unternehmen nicht alleine stehen darf: 
Befragter: (...) Ich glaube, es eignet sich nicht, um Unternehmensentscheidungen, äh, nur basierend auf dieser Quelle, vielleicht kommt das auch irgendwann mal, dass wir tatsächlich da, ähm, dass das so zunimmt. Oder ich meine, die (stockt) Digitalisierung schreitet ja (...) voran und letztendlich, was irgendwann mal zählen wird, sind nur noch die Daten. Ähm (...), aber die/ aktuell, glaube ich, sind einfach die Grenzen, das ist ein wichtiger Impulsgeber, aber es kann jetzt auch, äh, keine Marktforschung ersetzen, sondern kann einfach nur, ähm, es gibt uns/ es hält uns schnell einen Spiegel vor, den wir aber dann immer auch nochmal verifizieren müssen, ja. Aber es ist ein schneller Impulsgeber, den wir nutzen sollten, aber jetzt auch nicht überbewerten, ja. Das sind einfach so die, ähm, die Grenzen. Ja (I5/ S20-21/ Z45-5).

Social-Media-Analysen werden als Unterstützung für die Marktforschung gesehen. Die Befragten befinden, dass beide in eine Gesamtbetrachtung, ähnlich einem „Puzzle“ oder „Mosaik“, integriert werden sollten. Die Methode müsse heute ein fester Bestandteil der Marktforschung und ein Werkzeug im Methodenkoffer jedes Unternehmens sein:

Befragter: [. . . ] Es geht eher darum, aus Social Media und auch deren Analysen (...), wenn man intelligent in andere Auswertungen und Kunden-Klick-Pfade und User Flows und wie das alles heißt, einzuflechten und nicht einmal das Silo Social-MediaAnalyse zu haben, damit wir einmal Website-Daten zu haben, damit wir noch einmal Dealer-Daten, dann einmal irgendwie Probefahrt-Anfragen-Daten und (...) alles für sich in einem Silo liegt und keine Querverbindungen geschaffen werden. Also, ich glaube, die Herausforderungen, auch für uns im Marketing, wird sein, genau diese Querverbindungen aufzuzeigen und technisch hinzubekommen (I9/ S11/ Z40-47).

Die Ergebnisse von Social-Media-Analysen, die zwar Herausforderungen, aber ebenso Potentiale gegenüber anderen Methoden beinhalten, müssten nicht nur in einen Kontext mit den Ergebnissen anderer Methoden gestellt, sondern auch entsprechend interpretiert werden. Es gelte „Silos“ aufzubrechen, um ein Gesamtbild zu erzeugen und darüber letztlich Verständnis zu erlangen.

Social-Media-Analysen können sich die praktischen Anwender sowohl im Vorfeld von Marktforschung als auch im Nachgang dazu vorstellen. Monitoring und Listening seien dazu geeignet, zu prüfende Hypothesen zu generieren sowie Marktforschungsstudien zu „detaillieren“. Zwar könne die Methode etablierte Verfahren nicht ablösen, diese jedoch optimieren. Social-Media-Analysen sollen die ,harten Fakten' der Marktforschung mit Kundenmeinungen belegen; zur Illustration würden deskriptive Darstellungen durch die Methode um ,weiche Faktoren“ ergänzt. Umgekehrt gelten Social-Media-Analysen bei den Praktikern als geeignet, um neue, bislang unbekannte Themen in einem ersten Schritt explorativ zu erkunden. Basierend darauf könnten die Ergebnisse anschließend über Quantifizierung, erhärtet‘ werden. 
Social-Media-Analysen werden ferner oftmals dann durchgeführt, wenn quantitative Marktforschung die benötigten Detailinformationen nicht liefert, zu „oberflächlich" bleibt und mangels Tiefe die gesuchten Erklärungen nicht generiert. Hier zeigt sich erneut, dass die praktischen Anwender offenbar nicht dazu in der Lage sind, mehr Erkenntnisse als explizit verwertbare Hinweise aus den Daten zu ziehen. Ihrem Unternehmen mangelt es an Deutungskompetenz ebenso wie an den Ressourcen für Interpretationen; beide Faktoren bedingen sich gegenseitig. Der explorative Charakter von Social-Media-Analysen ist bei dem Automobilhersteller nicht legitimiert. Um die Ergebnisse der Methode zu verstehen und Erkenntnisse daraus ableiten zu können, sind Deutungen aber unerlässlich. Dies wurde ebenso längst erkannt wie die Tatsache, dass über qualitative Studien gewonnene Erkenntnisse unverzichtbar sind:

Befragter: //[A]lso (stockt) konkret, wenn wir über die Automobilindustrie, äh, sprechen, äh, wir haben ja immer wieder die Situation, dass in einer quantitativen Untersuchung ein Ergebnis rauskommt [. . . ]. Ich erinnere mich (atmet ein) an einen Fall, wo das Öffnen des Kofferraums kritisiert worden ist, die Ingenieure haben schon angefangen, äh, an den Federn rum zu schrauben, um die Schließkräfte, äh, zu reduzieren, aber eigentlich hat es dann mit daran gelegen, dass die Leute, äh, die Anbringung des Knopfes am Signalgeber, also am Schlüssel, äh, ungünstig platziert fanden. Und so was erfährt man dann eben im Zweifelsfall nur, wenn man qualitativen Input zu einem quantitativen Ergebnis hat. Sonst kann man sich halt komplett neben den Stuhl setzen (I18/ S3/ Z28-43).

Zwar wird mitunter die Bedeutung qualitativer Forschung und explorativen Vorgehens herausgestellt, zugleich aber deutlich gemacht, dass dies noch nicht in der ,Logik' des Automobilherstellers angekommen ist. Vorerst seien auch die Ergebnisse von Social-Media-Analysen in ein Gesamtbild zu integrieren. Ferner gelte es, den Kontext ihrer Entstehung zu beachten. Beide Herausforderungen hängen mit der Deutungskompetenz zusammen, die es erst noch zu entwickeln gilt. Ohne diese wird das Unternehmen - um im Bild zu bleiben - weder ein „Puzzle“ noch ein „Mosaik“ fertigstellen können.

Indem er nahezu ausschließlich fakten- und vergangenheitsorientiert ,denkt", folgt der deutsche Automobilhersteller seiner eigenen ,Logik'. Mit seinem Denkstil läuft er den Prinzipien qualitativer Forschung und explorativen Vorgehens zuwider. Social-Media-Analysen werden in dem betrachteten Unternehmen delegitimiert; das wird zum einen über die Diskreditierung einzelner Praktiker der Methode ausagiert, zum anderen über die ,Depotentialisierung ' des gesamten Verfahrens. Vor allem dann, wenn es anderen Methoden, wie Ansätzen klassischer Marktforschung, gegenübergestellt wird. 
Open Access Dieses Kapitel wird unter der Creative Commons Namensnennung 4.0 International Lizenz (http://creativecommons.org/licenses/by/4.0/deed.de) veröffentlicht, welche die Nutzung, Vervielfältigung, Bearbeitung, Verbreitung und Wiedergabe in jeglichem Medium und Format erlaubt, sofern Sie den/die ursprünglichen Autor(en) und die Quelle ordnungsgemäß nennen, einen Link zur Creative Commons Lizenz beifügen und angeben, ob Änderungen vorgenommen wurden.

Die in diesem Kapitel enthaltenen Bilder und sonstiges Drittmaterial unterliegen ebenfalls der genannten Creative Commons Lizenz, sofern sich aus der Abbildungslegende nichts anderes ergibt. Sofern das betreffende Material nicht unter der genannten Creative Commons Lizenz steht und die betreffende Handlung nicht nach gesetzlichen Vorschriften erlaubt ist, ist für die oben aufgeführten Weiterverwendungen des Materials die Einwilligung des jeweiligen Rechteinhabers einzuholen.

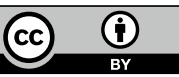

